\title{
Post-abortion care in Pakistan: A national study
}

\author{
Zeba Sathar \\ Population Council \\ Susheela Singh \\ Zakir Hussain Shah \\ Gul Rashida \\ Population Council \\ Iram Kamran \\ Population Council
}

See next page for additional authors

Follow this and additional works at: https://knowledgecommons.popcouncil.org/departments_sbsr-rh

Part of the Demography, Population, and Ecology Commons, Family, Life Course, and Society

Commons, International Public Health Commons, Maternal and Child Health Commons, Obstetrics and Gynecology Commons, and the Women's Health Commons

How does access to this work benefit you? Let us know!

\section{Recommended Citation}

Sathar, Zeba, Susheela Singh, Zakir Hussain Shah, Gul Rashida, Iram Kamran, and Kanwal Eshai. 2013.

"Post-abortion care in Pakistan: A national study." Islamabad: Population Council. 


\section{Authors}

Zeba Sathar, Susheela Singh, Zakir Hussain Shah, Gul Rashida, Iram Kamran, and Kanwal Eshai 



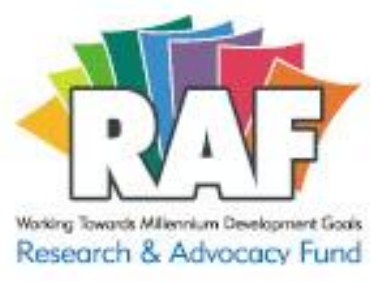

\section{Post-Abortion Care in Pakistan:}

\section{A National Study}
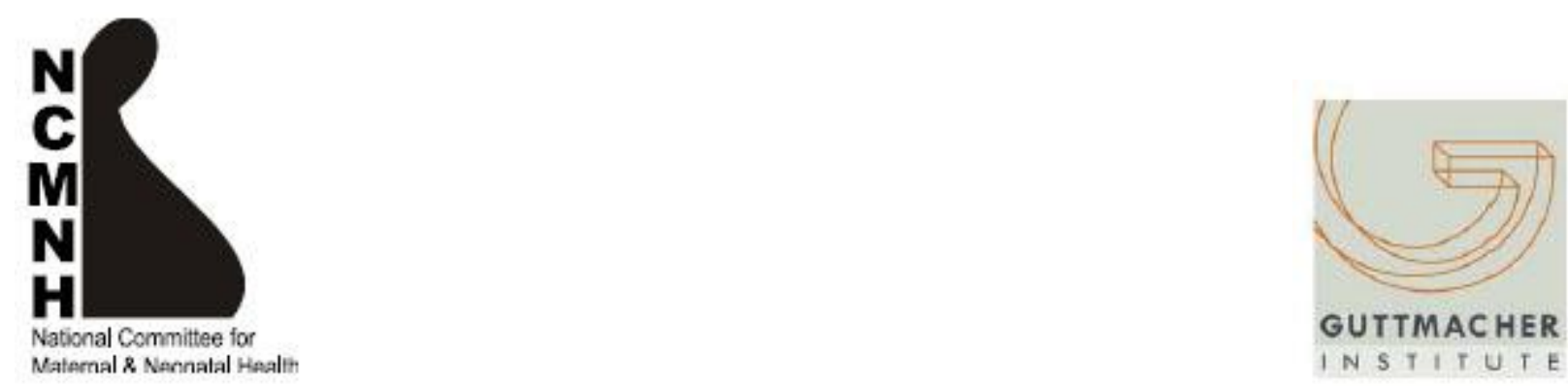


\section{(2) Population Council}

The Population Council confronts critical health and development issues-from stopping the spread of HIV to improving reproductive health and ensuring that young people lead full and productive lives. Through biomedical, social science, and public health research in 50 countries, we work with our partners to deliver solutions that lead to more effective policies, programs, and technologies that improve lives.

C 2013 The Population Council, Inc.

\section{Declaration}

"I have read the report titled "Post-Abortion Care in Pakistan: A National Study", and acknowledge and agree with the information, data and findings contained".

Dr.Zeba A. Sathar

Country Director, Population Council Islamabad

\section{Disclaimer:}

This document is an output from a project funded by the UK Department for International Development (DFID) and the Australian Agency for International Development (AusAID) for the benefit of developing countries.

The views expressed and information contained in it are not necessarily those of or endorsed by DFID, AusAID or the Maternal and Newborn Health Programme - Research and Advocacy Fund (RAF), which can accept no responsibility or liability for such views, for completeness or accuracy of the information, or for any reliance placed on them. 


\section{Table of Contents}

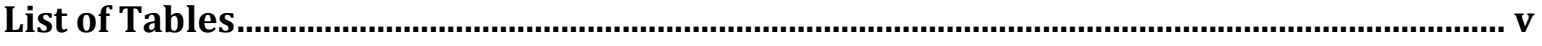

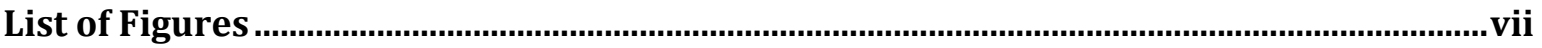

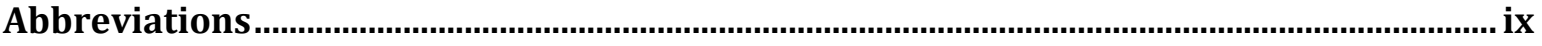

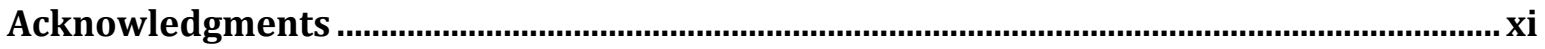

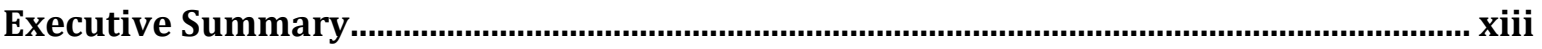

Chapter 1 Introduction

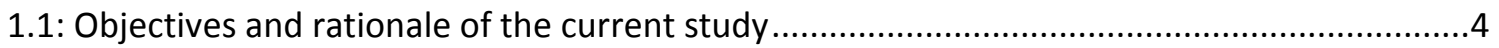

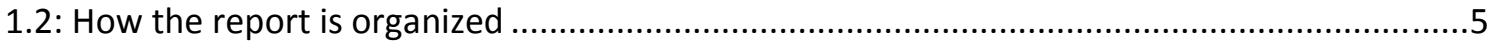

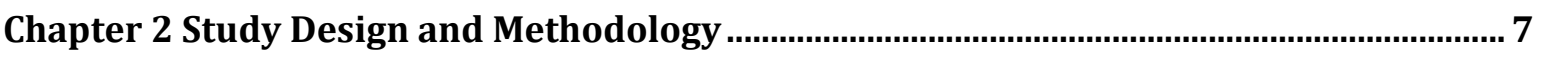

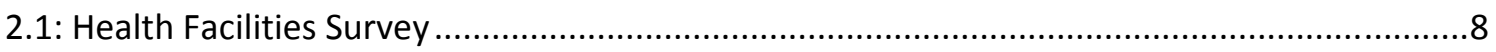

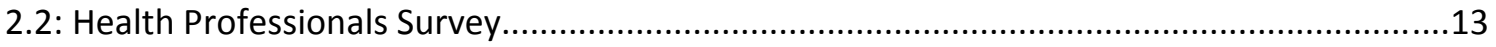

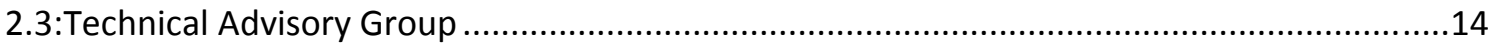

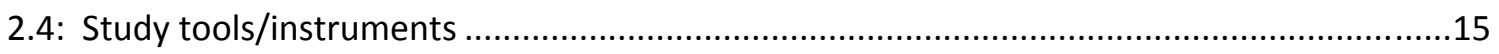

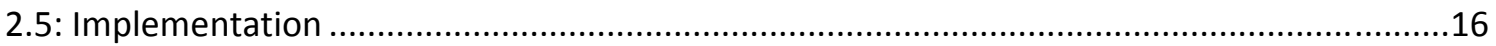

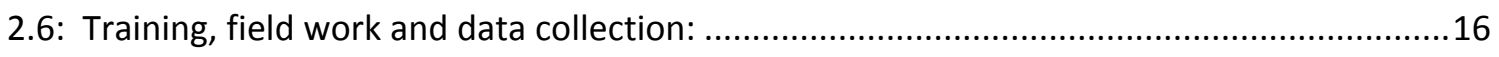

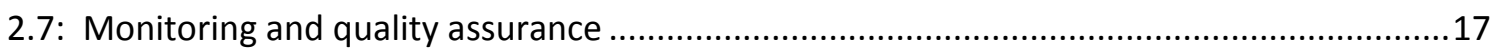

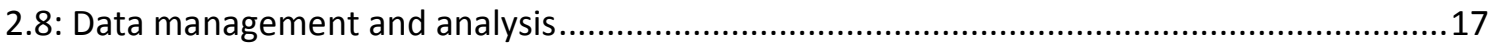

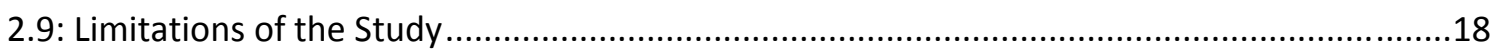

Chapter 3 The Practice and Health Consequences of Induced Abortion Among

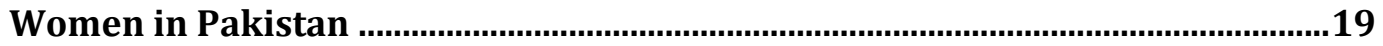

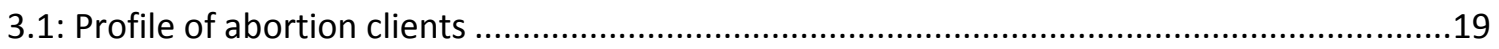

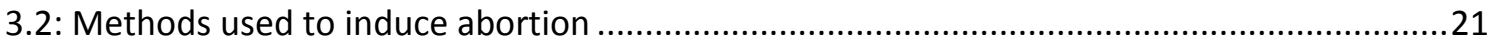

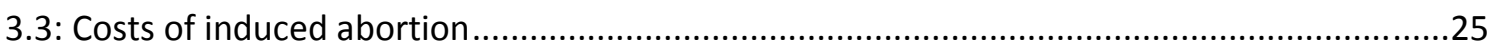

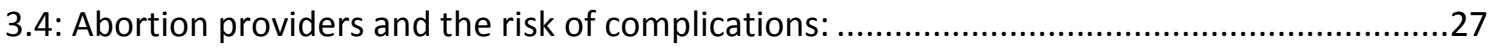

Chapter 4 Post-Abortion Care Patients and the Services they Receive ................................33

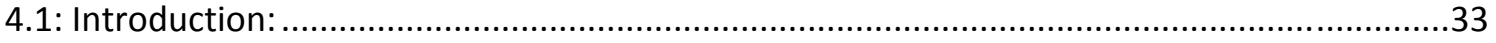

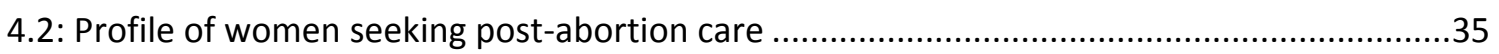

4.3: The likelihood that women with complications can obtain medical care .............................37

4.4: Availability and capacity of PAC services in the public and private sectors ..........................38

4.5: Procedures and services used in the treatment of abortion-related complications...............38

Chapter 5 Post-Abortion Care Service Statistics............................................................ 49

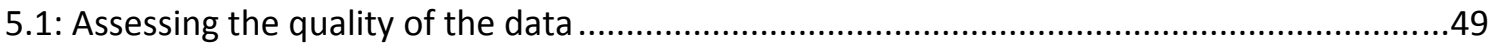

5.2: Differentials in annual PAC caseload by type of facility and by health sector ......................50

5.3: Differentials in annual PAC caseload by province ...............................................................53 
5.4: Treatment for post-abortion complications at Public-sector facilities between 2002-2012

Chapter 6 Family Planning Counselling and Services: An Important Aspect of PostAbortion Care

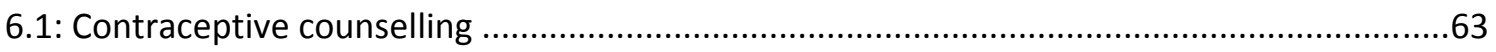

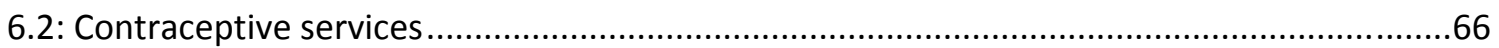

6.3: Provider attitudes to PAC, contraception and induced abortion .......................................68

Chapter 7 Barriers in Accessing Post-abortion Care: Women, Men and Providers'

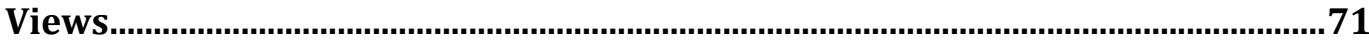

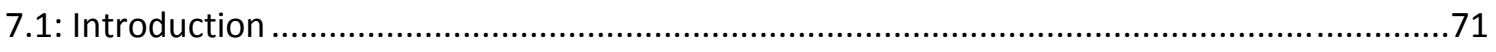

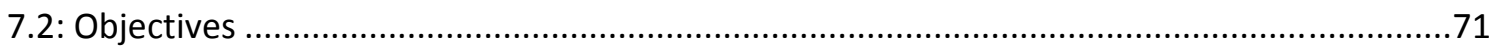

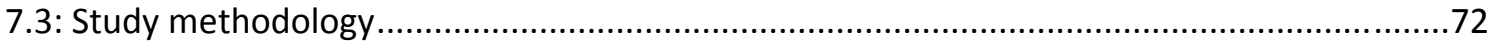

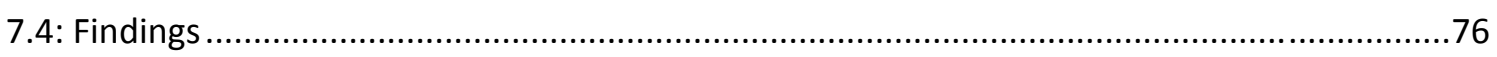

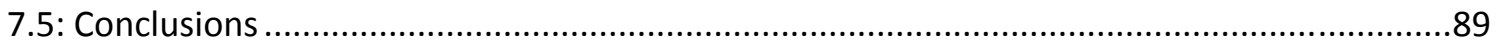

Chapter 8 Summary of Key Findings and their Implications for Policies and

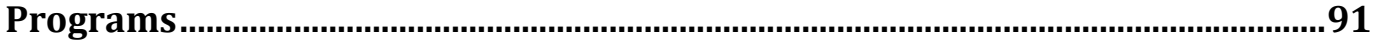

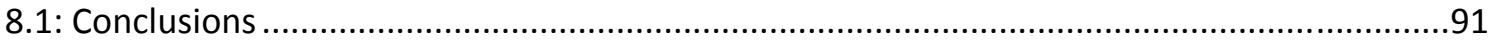

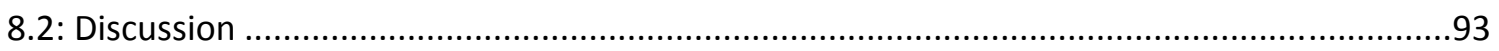

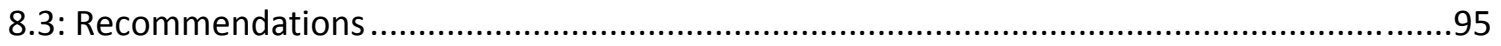

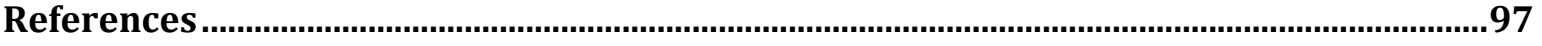

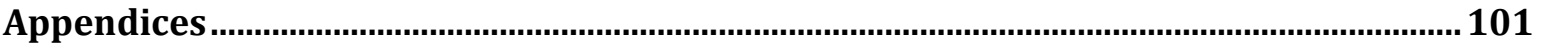

Annex 1: Ranking of districts according to income poverty at the district level .......................101

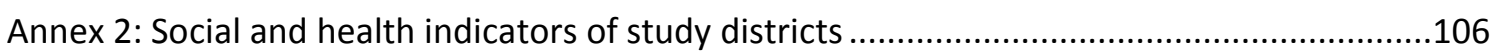

Annex 3: Members of the technical advisory group and study team.......................................107

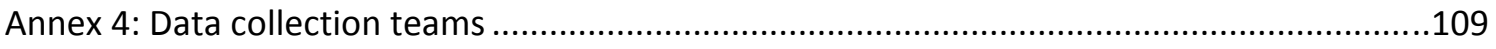

Annex 5a: Health facility statistics on post-abortion care ......................................................110

Annex 5b: Number of facilities recording statistics on post-abortion complications ................110

Annex 6: Annual caseload of post-abortion complications by sector, type of facility and

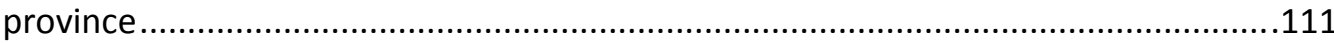

Annex 7: Application of weights to data obtained from public- and private-sector health

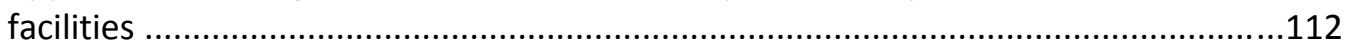

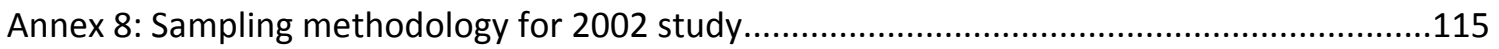

Annex 9: Social and health indicators of study districts-2002 ..............................................117

Annex 10: Standard errors and 95\% confidence intervals for major tables............................118

Annex 11: Graphical presentation and narrative of the framework approach used in the analysis. 


\section{List of Tables}

Table 2.1: Selection of teaching hospitals by sector....................................................................10

Table 2.2: Sampling of public-sector health facilities, by province .....................................................11

Table 2.3: Selection of private non-teaching health facilities, Health Facilities Survey, 2012 ..........12

Table 2.4: Number of health facilities included in the Health Facilities Survey, 2012.......................13

Table 2.5: Number and category of interviewed health professionals, by province, Health

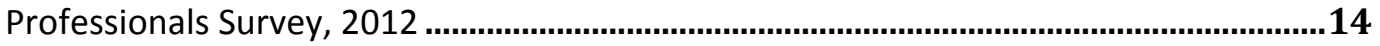

Table 2.6: Characteristics of respondents, Health Professionals Survey, 2012 .................................14

Table 3.1: Characteristics of women most commonly perceived as seeking termination of unwanted pregnancy, Health Professionals Survey, 2002 and 2012 ................................20

Table 3.2: Methods used by service providers to terminate an unwanted/unplanned pregnancy, as perceived by respondents, Health Professionals Survey, 2002 and 2012

Table 3.3: Methods most commonly used by women who perform their own abortions as perceived by respondents, Health Professionals Survey, 2002 and 2012

Table 3.4: Mean and median cost* of a first-trimester abortion, by type of provider, according to residence and economic status of women as perceived by respondents, Health Professionals Survey, 2012

Table 3.5: Proportion of women having abortions likely to experience complications, by type of abortion provider, according to women's residence and economic status, as

perceived by respondents, Health Professionals Survey, 2002 and 2012

Table 4.1: Characteristics of women most commonly perceived as attending health facilities for treatment of post-abortion complications, Health Facilities Survey, 2002 and 2012.

Table 4.2: Proportion of women experiencing abortion-related complications estimated by health professionals to seek medical treatment, by women's residence and economic status, Health Professionals Survey, 2012

Table 4.3: Proportion of health facilities with at least one female medical personnel on staff, by type of facility, Health Facilities Survey, 2012

Table 4.4: Proportion of facilities that do not have 24-hour coverage of staff, according to type of staff and type of facility, Health Facilities Survey, 2012

Table 4.5: Number of doctors and nurses who received training in various PAC-related procedures, by province, Health Facilities Survey, 2012.

Table 4.6: Number of doctors and nurses who received training in various PAC-related procedures, by type of facility, Health Facilities Survey, 2012.

Table 4.7: Proportion of facilities with functioning PAC equipment, by type of facility, Health

Facilities Survey, 2012 
Table 4.8: Proportion of health facilities equipped with standard post-abortion care (PAC) supplies, by type of facility, Health Facilities Survey, 2012.

Table 5.1: Annual numbers of PAC patients treated as out and in-patients, by health sector and type of facility, Health Facilities Survey, 2012.

Table 5.2: Annual mean caseload of PAC (out and in-patients) by type of facility, health sector and province, Health Facilities Survey, 2012

Table 5.3: Annual number of post-abortion complication (out and in-patients) cases treated in public health facilities, by province, Health Facilities Survey, 2002 and 2012

Table 5.4: Mean annual number* of public-sector PAC (out and in-patients) cases, by type of facility and by province, Health Facilities Survey, 2002 and 2012

Table 5.5: Number and rate of women treated for post-abortion complications per 1,000 women aged 15-49 in 2012, by province, Health Facilities Survey, 2012.

Table 5.6: Rate of women treated for abortion complications in public-sector health facilities per 1,000 women 15-49, by province, Health Facilities Survey, 2002 and 2012..............61

Table 6.1: Proportion of health professionals who consider post-abortion counselling on selected topics to be adequate, Health Professionals Survey, 2012.

Table 6.2: Percent of facilities reporting that they commonly offer specific types of contraceptives to post-abortion patients, by type of health facility, Health Facilities Survey, 2012

Table 6.3: Percent of facilities that refer clients to other health institutions, by type of facility, Health Facilities Survey, 2012

Table 7.1: District wise distribution of respondents by type of interview (IDIs, FGDs and informal discussions)

Table 7.2: Socio-demographic profile of the respondents

Table A1: Health facility statistics on post-abortion care

Table A2: Annual caseload* of post-abortion complications (out-and in-patients) by sector, type of facility and province, 2012(hospitals statistics)

Table A3: Weights of health facilities by sector and by number of beds

Table A4: Mean numbers of annual PAC patients treated as out and in-patients, by health sector and type of facility, Health Facilities Survey, 2012

Table A5: Annual numbers of PAC patients treated as out and in-patients, by health sector Health Facilities Survey, 2012

Table A6: Annual number of post-abortion complications (out and in-patients) cases treated in health facilities, by province, Health Facilities Survey 2012 


\section{List of Figures}

Figure 2.1: Geographical distribution of study districts

Figure 3.1: The two most common methods used to terminate an unwanted/unplanned pregnancy as perceived by respondents, Health Professionals Survey, 2002 and 2012

Figure 3.2: The two methods most commonly used by women who perform their own abortions as perceived by respondents, Health Professionals Survey, 2002 and 2012

Figure 3.3: Average cost* of a first-trimester abortion, by type of provider as perceived by respondents, Health Professionals Survey, 2002 and 2012.

Figure 3.4: The type of provider used by urban women for obtaining abortions, by the women's economic status as perceived by respondents, Health Professionals Survey, 2002 and 2012.

Figure 3.5: The type of provider used by rural women for obtaining abortions, by the women's economic status as perceived by respondents, Health Professionals Survey, 2002 and 2012

Figure 4.1: Proportion of women experiencing abortion-related complications estimated by health professionals to obtain post-abortion care (PAC), by women's residence and economic status, Health Professionals Survey, 2002 and 2012.

Figure 4.2: Proportion of respondents at public health facilities reporting the various procedures that may be used to treat post-abortion complications, Health Facilities Survey, 2002 and 2012

Figure 4.3: Percent distribution of women treated for post-abortion care (PAC), by type of procedure, Health Facilities Survey, 2012.

Figure 4.4: Proportion of respondents saying which treatments would be best recommended for treating a first-trimester PAC case, Health Facilities Survey, 2012.

Figure 4.5: Proportion of health facilities employing specific types of staff, Health Facilities Survey, 2012.

Figure 4.6: Proportion of public and private facilities that do not have 24-hour coverage, by type of medical staff, Health Facilities Survey, 2012

Figure 4.7: Proportion of doctors who have received training in various PAC-related procedures, by duration of their training, Health Facilities Survey, 2012

Figure 5.1: Total annual number of PAC patients treated in health facilities, by type of facility, Health Facilities Survey, 2012.

Figure 5.2: Annual caseload of PAC patients, by public and private sector and by province, Health Facilities Survey, 2012 
Figure 5.3: Mean annual number of PAC cases per public health facility, by province, Health Facilities Survey, 2002 and 2012.

Figure 5.4: Mean annual number of out-and in-patient PAC cases in public-sector facilities, by type of facility, Health Facilities Survey, 2002 and 2012.

Figure 6.1: Proportion of public and private-sector health facilities providing post-abortion counselling on birth spacing and contraception, by type of facility, Health Facilities Survey, 2012

Figure 6.2: Proportion of women treated in health facilities who receive post-abortion counselling on one or more of a range of health-related issues, * by sector and type of facility, Health Facilities Survey, 2012 .

Figure 6.3: Proportion of health facility respondents of who agree with various statements regarding post-abortion care, Health Facilities Survey, 2012.

Figure 6.4: Proportion of health professionals who agree with various statements regarding post-abortion care, Health Professionals Survey, 2012

Figure 6.5: Proportion of health facility respondents who agree with various attitudes about the practice of abortion, Health Facilities Survey, 2012

Figure 6.6: Proportion of health professionals who agree with various attitudes about the practice of abortion, Health Professionals Survey, 2012 ...

Figure 6.7: Attitudes toward the practice of induced abortion, by type of health professional, Health Professionals Survey, 2012 


\section{Abbreviations}

\begin{tabular}{|c|c|}
\hline $\mathrm{BHU}$ & Basic Health Unit \\
\hline $\mathrm{Cl}$ & Confidence Interval \\
\hline CPR & Contraceptive Prevalence Rate \\
\hline CSPro & Census and Survey Processing System \\
\hline $\mathrm{D} \& \mathrm{C}$ & Dilatation and Curettage \\
\hline D\&E & Dilatation and Evacuation \\
\hline DHQ & District Headquarters Hospital \\
\hline DHS & Demographic and Health Survey \\
\hline EVA & Electrical Vacuum Aspiration \\
\hline FGD & Focus Group Discussion \\
\hline FHT & Family Health Technician \\
\hline FP & Family Planning \\
\hline FP \& PHC & Family Planning and Primary Health Care \\
\hline FWW/ FWC & Family Welfare Worker/Family Welfare Counsellor \\
\hline GIS & Geographical Information System \\
\hline GP & General Practitioner \\
\hline GYN/OBS & Gynaecology and Obstetrics \\
\hline HFS & Health Facilities Survey \\
\hline HMIS & Health Management Information System \\
\hline HPS & Health Professionals Survey \\
\hline ICPD & International Conference on Population and Development \\
\hline ICU & Intensive Care Unit \\
\hline IDI & In-Depth Interview \\
\hline IRB & Institutional Review Board \\
\hline IUCD & Intrauterine Contraceptive Device \\
\hline IV & Intra Venous \\
\hline KPK & Khyber Pakhtunkhwa \\
\hline LHV & Lady Health Visitor \\
\hline LHW & Lady Health Worker \\
\hline MBBS & Bachelor of Medicine/ Bachelor of Surgery \\
\hline MDGs & Millennium Development Goals \\
\hline
\end{tabular}




\begin{tabular}{|c|c|}
\hline MHT & Medical Health Technician \\
\hline $\mathrm{MNCH}$ & Maternal, Newborn and Child Health \\
\hline MVA & Manual Vacuum Aspiration \\
\hline $\mathrm{NCMNH}$ & National Committee for Maternal \& Neonatal Health \\
\hline NIPS & National Institute of Population Studies \\
\hline NPFP \& PHC & National Program for Family Planning and Primary Health Care \\
\hline $\mathrm{OP}$ & Oral Pills \\
\hline PAC & Post-Abortion Care \\
\hline PBS & Pakistan Bureau of Statistics \\
\hline PDHS & Pakistan Demographic and Health Survey \\
\hline PMDC & Pakistan Medical and Dental Council \\
\hline PPIUCD & Postpartum Intrauterine Contraceptive Device \\
\hline PPHI & People's Primary Healthcare Initiative \\
\hline PRSP & Punjab Rural Support Programme \\
\hline QoC & Quality of Care \\
\hline $\mathrm{RH}$ & Reproductive Health \\
\hline $\mathrm{RHC}$ & Rural Health Centre \\
\hline SE & Standard Error \\
\hline SOGP & Society of Obstetricians \& Gynaecologists of Pakistan \\
\hline SOP & Standards of Practice \\
\hline TBA & Traditional Birth Attendants \\
\hline TFR & Total Fertility Rate \\
\hline THQ & Tehsil Headquarters Hospital \\
\hline WHO & World Health Organization \\
\hline WMO & Woman Medical Officer \\
\hline
\end{tabular}




\section{Acknowledgments}

Post-Abortion Care in Pakistan: A National Study is a project funded by the Maternal and Newborn Health Programme-Research and Advocacy Fund (RAF), and is implemented by the Population Council, Pakistan.The Population Council would like to express its gratitude to the Maternal and Newborn Health Programme-Research and Advocacy Fund for the funding for this study and Ms. Sarah Hall- Programme Manager, Dr.WajihaGhias- Manager Grants and Mr.ZahidMemon- Research Specialist, for their invaluable support throughout the study.

We are thankful to the four Provincial Health Departments, District Health Officers of the study districts, officers and staff of the surveyed public and private health facilities, for their facilitation and cooperation during data collection. We are also grateful to the staff of the Departments of Health, particularly the staff of the BHUs, and the Lady Health workers of the Provincial Program for Family Planning and Primary Healthcare, who helped us in identifying communities, recruiting respondents and arranging FGDs.

Apart from the authors and contributors at the Population Council, several experts in the field provided their valuable inputs in the preparation of this report. We were most fortunate to have benefited from the vast experience and insights of Dr.SadiquaN. Jafarey, President of the National Committee on Maternal, New born and Child Health (NCMNH), who is an undisputed authority on maternal health. Her valuable technical inputs, review and guidance were available to us at every stage of the implementation of this study and finalization of the report.

We acknowledge the role of Technical Advisory Group for their guidance and constructive inputs in development of the study tools and policy recommendations. We are grateful for the comments received from the review provided by the Research and Advocacy Fund. They were very useful in addressing remaining issues with the final report.

We particularly acknowledge the contribution of Ms. Deidre Wulf, from the Guttmacher Institute, who most painstakingly edited the report.

We would like to appreciate and acknowledge the contribution of our study team members comprising Dr.Sadaf Gul, Mr.Mumraiz Khan, Dr. Saleem Sheikh, Mr. Khan Mohammad, Ms.LubnaMehmood and Mr.UsmanAsif. We thank each of the members of the field research teams who put in outstanding efforts to collect the data on which this study is based. And we are thankful to the administration and finance and IT departments of the Population Council for their 
valuable support in the implementation of this national study. We thank Mr. Ali Ammad who formatted and finalized the layout of this report.

And finally, we owe special thanks to all the study participants, the Health Professionals and Health facility staff who spared their time to answer our questions and provided the useful information which will be utilized to influence the processes, policies and programs of maternal health care in each of the provinces of Pakistan. 


\section{Executive Summary}

This study was essentially driven by the need to take stock of changes that have taken place since the 2002 national study of 'Unwanted Pregnancy and Post-abortion Complications in Pakistan' (Population Council 2004). On all accounts, post-abortion complications remain a serious public health challenge, and assessing the availability of care in the country's public and private health facilities continues to be important for the design of reproductive health policies and programs a decade later. The objectives of the current study were twofold; first to collect information on prevailing practices of abortion and post-abortion care and, second, to gauge changes in the magnitude and quality of abortion-related complications and care during the last decade. The design follows that of the earlier study as far as possible, but the 2012 study obtains new information and expandsthe representation of private-sector facilities.

Two cross sectional surveys, a Health Professionals Survey and a Health Facility Survey, were conducted to interview selected health professionals and to survey sampled facilities across four provinces of Pakistan. A separate structured questionnairewas developed and face to face interviews were carried out by trained researchers.An additional qualitative component of the study included in-depth interviews (IDIs), focus group discussions (FGDs) and informal discussions with women, service providers and men in the community setting in five districts across the country.

In Pakistan as elsewhere, getting direct information from women about abortion and postabortion complications is difficult because of the stigma and reluctance attached to the answers. This study therefore relies on health professionals and on facility staff for responses on characteristics of women seeking abortion and post-abortion care and of women that present at their facilities. The methodological approach used in this study is based on an internationally accepted methodology developed by the Guttmacher Institute to approach the estimation of the incidence of abortion and post-abortion complications worldwide. It is the same approach utilized in the earlier study carried out in Pakistan by the Population Council in 2002.

The study shows that696,000 women with post-abortion complications present themselves annually in public and private health facilities. This number clearly imposes a heavy burden on the health system in Pakistan. The role of the private sector is particularly prominent; it is estimated totreatabout $50 \%$ morepost-abortion casesthan public health facilities do. This reliance on the private sector for post-abortion complication care appears to have increased over the past decade. This is largely because the private sector is much larger and has many more health 
facilities than in 2002.Nevertheless, public-sector facilities in all provinces report a higher average caseload of women with post-abortion complications than is found in private-sector facilities.

Between 2002 and 2012, the total number of post-abortion cases treated at public health facilities in the provinces of Punjab, Sindh, Khyber Pakhtunkhwa (KPK) and Balochistanincreased moderately from around 246,000 to 267,000 . With the increase in the country's population size during the last decade, the public sector treatment rate decreasedslightly, from 7 to 6 per 1,000 women of reproductive age.

The 2012 overall rate of women treated for abortion-related health complications in both the private and public sectors is 15 per 1,000 women aged 15-49. The rate is lower in KPK than inthe other three provinces surveyed. There have been increases in the proportion of women who received treatment as out-patients at public health facilities (especially in Sindh and Balochistan), which indicates a possible decrease in the severity of complications. While theaverage caseload at public health facilities declined during the last decade, it rose at public teaching hospitals and at rural health centres (RHCS).

There has been little change in the past decade inthe demographic characteristics of both women seeking induced abortion and those seeking post-abortion care (PAC). The majority of women seeking such services are married, aged 30 years or more, residents of rural areas, uneducated, poor and with five or more children. What has changed, however, are the methods commonly used to induce abortions. The use of unsafe and invasive methods such as laminaria sticks, IUCD and anti-malarial medication, while commonly reported in 2002, has declinedover the past decade. At the same time, the use of safer techniques such as MVA and EVA has increased. The use of misoprostol to induce abortions, which did not even show up in 2002, has become prominent. Nevertheless, recourse to the invasive D\&C method remains as frequent an abortion method in 2012 as in 2002.

The proportion of women who obtain abortions performed by doctors has increased. A large proportion of women in urban areasstill resort to TBAs/Dais/lay practitioners and LHVs/Nurses/Midwives, all providers associated with relatively high probabilities of complications. TBAs still remain the most available abortion providers for rural women. The costs to women of an induced abortion have nearly doubled over the last 10 years, and the relatively greatest increase has been among both rural and urban poor women paying the fees charged by LHVs/Nurses/Midwives and TBAs/Dais.

The reported proportions of women experiencing abortion-related complications who are likely to receive treatment in a health facility have also increased. Although the change is greatest for poor 
rural women than for any other group, still barely half of those who develop post-abortion complications are able to obtain treatment.

The most striking finding of the study is the increase in the use ofmisoprostol both to induce abortion and to treat post-abortion complications. Misoprostol is reported to be a leading technique in private teaching hospitals and inpublic-sector RHCs; public teaching hospitals and private health facilities other than teaching hospitals, however,are much less likely to use this method. The use of surgery to treat post-abortion complications has dropped, possibly as a result of a declines in the incidence of the moresevere types of complications (such as damage to the uterus and gut) reported in the 2002 study.

A majority of public and private facilities of all levels have D\&C sets in stock, but the availability of MVA and EVA kits is limited, especially in non-teaching facilities. The majority of facility respondents recommended medication abortion as the best procedure for treating first trimester PAC cases. However, a very small proportion considered vacuum aspiration as the recommended procedure for PAC, reflecting a possible lack of awareness about MVA and EVA. We recommend that misoprostol and MVA/EVA should be promoted as methods of PAC and that misoprostol should be included in the essential drugs list. Health facilities should be better equipped and their staff trained to provide better quality post-abortion care services using misoprostol and vacuum aspiration methods.

The level of post-abortion family planning (FP) counselling in both public and private health facilities offering PAC services is inadequate.There has been no improvement over the decade in the level of counselling reported, and, even more disappointingly, only half of health facilities provided women receiving PACwith contraceptives. Given the strong linkages between unwanted pregnancies, abortions and unmet need for family planning, this is perhaps one of the public health system's main shortcoming and a huge missed opportunity. We therefore recommend that family planning should be included in all medical curricula, from the undergraduate level to inservice trainings. Interaction, coordination and synergy between the departments of family planning and gynaecology/obstetrics in hospitals should be strengthened to ensure that contraceptive supplies and FP counselling become standard operating procedure both following a delivery (postpartum) and after women have received post-abortion care. 



\section{Chapter 1 Introduction}

\section{Induced abortion in Pakistan}

With a population of 180 million, Pakistan is the sixth most populous country in the world. According to the 2006-2007 Pakistan Demographic and Health Survey (PDHS), the total fertility rate (TFR) declined from 5.4 children per woman in 1990-1991 to 4.1 children16 years later. Women with a higher level of education had 2.5 fewer children, on average, than uneducated women.Fertility preferences play an important role in estimating unmet need for family planning andthe future fertility of a particular population. Data show that in Pakistanmore than $50 \%$ of currently married women aged 15-49 did not want another child at all. Besides number of living children, future fertility preferences were also strongly linked with the sex of the children. Between 1990-1991 and 2006-2007, the proportion of women who wanted to limit their family size increased from $40 \%$ to $52 \%$. Furthermore, women living in urban areas preferred smaller families than did rural women. In 2006-2007, almost one in four births in Pakistan (24\%) was unintended.

The PDHS carried out over a number of years show that contraceptive use has increased substantially since the mid-1980s; however, for the last couple of years the rate has been stagnating. Multiple factors, including weak support from the health sector and a delivery gap between the community and service provision, are at play in the lack of increase in CPR. The current contraceptive prevalence rate (CPR) in Pakistan is low-30\% among married women aged $15-49$ and $25 \%$ of thesesame women have an unmet need for contraception. Of these, $11 \%$ want to space births while the remaining $14 \%$ want to stop childbearing altogether. Contraceptive use is largely dependent upon a woman's age and parity, and the highest level of use (42\%) is found among women aged 40-44 years. Some of the reasons for non-useinclude women's weak autonomy in household decision-making and the economic costs of contraception ${ }^{1}$. The current high level of unmet need for family planning, low level of contraceptive prevalence and high level of unwanted and unintended pregnancies are key drivers of abortion levels in Pakistan, and thus of theadverse health consequences of unsafe abortion, which can encompass a range of serious health complications ${ }^{2,3}$. Morbidity resulting from unsafe abortion has a substantial impact both on women's health and on the health care system in Pakistan. Since poor women and couples are those least able to obtain contraception and most likely to have unintended pregnancies, they are 
also more likely to resort to abortion to achieve their fertility goals $s^{4,5}$. The high level of unmet need for family planning is no doubt the main reason why, despite the illegality of the procedure, the abortion rate in Pakistan is so high. ${ }^{4}$

A national study carried out in 2002 by the Population Council and the Guttmacher Institute projected that 890,000 induced abortions took place in Pakistan in 2002,7. Of every 100 pregnancies occurring, 14 ended in induced abortion. Abortion levels appeared to be considerably higher in two of the four provinces with lower levels of contraceptive use: a rate of 38 abortions per 1,000 women in Balochistan, and of 37 per 1,000 in Khyber Pakhtunkhwa (KPK),compared to25 per 1,000 and 31 per 1,000, respectively, in Punjab and Sindh ${ }^{6,7}$.

Unsafe abortion continues to be an important contributor to high levels of maternal mortality in Pakistan, even though its role is probably underreported. The2002 study estimated that in a oneyear period, 197,000 women were treated in public health facilities or private teaching hospitals for complications resulting from unsafe induced abortion. Many other women who experiencedabortion-related complications requiring treatment never reacheda healthcare facility, and their number, according to the study, is several times higher than that of women who did receive care. Poorer women and those living in rural areas had the lowest chances of obtaining the care they needed.

Various small-scale ${ }^{8,9,10}$ studies in Pakistan have found that the majority of women who seek abortion are poor, uneducated, married, are relatively older and have five or more children. Abortion is seen as a quick, easy, and tolerable method of fertility regulation for women who experience an unintended pregnancy because of the unavailability of contraceptives, method failure or the discontinuation of a method in response to actual or perceived side effects.

High levels of unwanted pregnancy ${ }^{11}$ lead women to seek clandestine abortions performed by unskilled providers using unsafe methods, which can often result in medical complications. Until 1990, abortion in Pakistan was regulated by a century-old Penal Code 1860 developed for India by the British colonial government. This law remained in force in Pakistan following Independence. Under the Code, abortion was a crime unless performed in good faith in order to save a pregnant woman's life.Pakistan revised its abortion law, reformulating a number of its provisions to conform to the principles of Islamic law. The revised law came into effect provisionally in 1990 and became permanent law in 1997. Abortion offences are currently divided into two categories depending on the stage of pregnancy during which the abortion is performed. The new law in Pakistan allows abortion in the early stages of pregnancy to save the life of the woman or to provide necessary treatment but it is silent on the issues of rape, incest and fetal abnormalities ${ }^{12,13,14}$. Moreover, there is weak understanding about post-abortioncare (PAC) among public-sector health providers and managers. 
In Pakistan, postpartum haemorrhage, antepartum haemorrhage, puerperal sepsis, obstructed labour, eclampsia and complications related to unsafe abortion have been identified as the leading causes of maternal death ${ }^{15}$. In Pakistan, 6\%of maternal deaths resulted from the complications of unsafe abortion (sepsis or haemorrhage) according to the 2006-2007 PDHS ${ }^{4}$. A 1990-2001 study conducted in a hospital setting found that $11 \%$ of maternal deaths during the study period were due to complications of unsafe induced abortion performed by untrained service providers in unhygienic conditions. Thus, unsafe abortions and theircomplications are responsible for a sizeable proportion of maternal deaths ${ }^{16}$.

A large number of women experiencing post-abortion complications in Pakistan remain invisible because they do not present themselves at hospitals for treatment. This is particularly true for poor rural women who lack access to a medical facility,or cannot afford the costs of treatment ${ }^{6}$. Abortions are expensive: the cost of the procedure averages 1,145 rupees (the average household income in Pakistan is 25,679 rupees) ${ }^{17}$, and on top of this the costs of transportation and medicines are often difficult for families to cover. Delay in seeking treatment for post-abortioncare is common, and only one-quarter of women obtaining care also receive family planning counselling 5 .

Since the 1994 International Conference on Population and Development (ICPD), post-abortion care (PAC) has gained importance as a vital aspect of good reproductive health programs. About180countries came to a joint consensus that,regardless of the legal status of abortion, "in all cases, women should have access to quality services for management of complications arising from abortion. Post- abortion counselling, education and family planning services should be offered promptly, which will help to avoid repeat abortions ${ }^{18}$." PAC is defined as including emergency treatment for complications of spontaneous or unsafe abortions, family planning/birth spacing counselling and services and alinkage between emergency abortion treatment services and comprehensive reproductive health care. Better PAC services can play a vital role in addressing the issue of unsafe abortion and can significantly reduce maternal mortality and morbidity. Even in a country withrestrictive abortion laws, PAC services should be uncontroversial to implement because their aim is totreat women after they have experienced an unsafe induced or spontaneous abortion ${ }^{19}$.

Given the strong evidence on the relationshipbetween unsafe abortion and threats to women's reproductive health, the Government of Pakistan has ramped up efforts to reduce abortionrelated maternal mortality and morbidity, including steps to improve access to family planning services. The October 2009 Karachi Declaration on Scaling up MNCH-FP Best Practices in Pakistan clearly pledges ${ }^{20}$, along with the provision of family planning services, to ensure the "inclusion of the practice of post-abortion care in policies, guidelines, protocols and standards for health 
facilities at the national level." These policy commitments and the training and advocacy activities that have been taking place over the past decade are expected to have had an impact on the coverage and quality of post-abortion care now being offered in Pakistan.

\section{1: Objectives and rationale of the current study}

The most recent study on unwanted pregnancy and post-abortion complications was carried out by the Population Council in 2002, and provided national-level estimates of the prevalence of induced abortion, as well as information on abortion providers, abortion methods and the conditions under which induced abortions were being performed. The present study was conducted to assess whether and how these conditions have changed over the past 10 years, including changes in abortion practices, abortion-related complications and the provision of post-abortion care, or any changes in regional patterns. In addition, the study aims to provide a more in-depth understanding of gaps and needs in PAC services. The last decade has seen some major changes in the context of abortion issues and overall health policy shift at the national level. For example the sensitivity around the issue of abortion has decreased and initiatives have been taken to introduce and use safer methods for post-abortion care(MVA and misoprostol). Through a Constitutional amendment, the Federal Ministries of Health and Population have been abolished and their functions have been devolved to the respective provincial departments. Each province is developing its own Health and Population Strategies that are being translated into their respective policies. At this time it was considered important to generate recent evidence to inform policy and programs about in order to influence and improve PAC in Pakistan.

More specifically, the study assesses changes in the conditions and practice of unsafe abortion in Pakistan, and provides updated national and provincial estimates on the number of facility-based treatments for post-abortion complications. The study utilizes a complete list of public-sector facilities that provide post-abortion care and also examines the provision of post-abortion care by private-sector facilities, using a newly available listing of these facilities for 35 of Pakistan's approximately $114^{*}$ districts.

The research focuses on two major questions: What is the current coverage and quality of postabortion care in the public and private sector? And secondly, how has this changed over the last decade? This information allows us to assessremaining gaps in the provision of post-abortion care in 2012 and to make recommendations on how to close these gaps.Other more specific questions the study examines are whether poor and rural women are more or less likely to receive care for

\footnotetext{
*There are 139 districts in Pakistan; the four provinces sampled in this study cover 114 of the total number of districts. We did not conduct the survey in Azad Jammu and Kashmir (AJK) province or in Federally Administered Tribal Areas.
} 
post-abortion complications in 2012 compared to 2002; and whether post-abortion care is being offered by a higher proportion of public-sector facilities in 2012 than in 2002 . We assess private and public-sector facilities in terms of the quality of their post-abortion care services in 2012, their physical and staffing capacity, their adherence to WHO-recommended standards of practice (SOP), and their use of less-invasive procedures such as MVA (an existing and recommended method) and medication abortion, as compared to the more traditionally used method-dilatation and curettage (D\&C). The researchers also looked at the willingness of staff to provide treatment to abortion patients and providers' attitudes toward the issues of induced abortion, post-abortion care and contraception-an important aspect ofquality of care.

The results of the study will help measureexisting gaps in the trainingof middle- or low-level providers in safer PAC techniques, a strategy that would help reduce morbidity among poorer women. The adoption of relatively inexpensive techniques-such as manual vacuum aspiration (MVA) in place of D\&C-as preferred methods of treatment could reduce the level and severity of morbidities, lead to more equitable health outcomes, and enable women living in remote areas to receive the kind of high-quality care that is currently available exclusively in larger facilities or comparatively more urban and richer regions of Pakistan.

The study findings should spur renewed attention to the issue of health complications resulting from unsafe abortionandhelp pinpointdeficits in post-abortion care, particularly forpoorer and rural women. The findings of the study will be presented to policy makers in the hopesof bringing about appropriateprogram and policy interventions, such as improved quality of PAC services through the better and wider training of providers and the provision of well-equipped facilities.

\section{2: How the report is organized}

Chapter 1 provides the global and local context for the present study and highlights the need for data to identify priority actions to be taken at the national level to reduce the incidence of unsafe abortion. It gives an overview of the issue of abortion in Pakistan, and compares it with the situation in developing countries and the developed world. Chapter 2 describes the study design and overall methodology. It describes the sampling procedures used in the surveys of health facilities and of health professionals, and the data collection tools and methodology. The training of medical researchers prior to the start of field work, some major issues faced during data collection, and procedures for data management and analysis are also discussed.Chapter 3 describes, as reported by health professionals, the various methods used by women themselves to induce their own abortions and the pregnancy termination servicesused by providers. It also discusses the typical profile of clients seeking pregnancy termination anddescribes typical abortion providers among urban and rural, poor and non-poor women, comparing these findings 
with those of the earlier 2002 study. Chapter 4 discusses health professionals' reports aboutthe characteristics of women experiencing post-abortion complications, the proportion of abortions likely to result in complications and the type and level of health facilities that womenneeding treatment go to. This chapter also describes the preparedness of services to provide postabortion care, the type of staff available and the various medical procedures used to treat women suffering the health complications of unsafe abortions. Chapter 4 also examines the status of staff trained in procedures to manage abortion-related complications. Chapter 5 analyses the burden on the health delivery system, both public and private, from treating patients with post-abortion complications. It presents the average annual number of cases treated as out-patients and as inpatients, by province and type and level of facility. The findings are compared with those of the 2002 study to show changes in caseloads across the four major provinces of Pakistan.In Chapter 6 we analyse the proportion of facilities providing various types of family planning methods and describe the attitudes of health professionals and service providers toward the role of contraception and post-abortion family planning counselling services. Chapter 7 explores the demand side of the issue of post-abortion care by assessing barriers and challenges women face in seeking care in a variety of settings mainly in rural Pakistan where there is a greater lack of services. Chapter 8 concludes the report by discussing the overall lessons learnt from the study and makes recommendations for future actions at the program and policy level. 


\section{Chapter 2 Study Design and Methodology}

The design of the study was based on an earlier approach used by the Population Council in its study 'Unwanted Pregnancy and Post-Abortion Complications in Pakistan' in 2002 ${ }^{21}$.Following a similar design to the one used in the earlier study permitsrobustcomparisons over time. Using an internationally accepted estimation technique,the Guttmacher Institute has carried out similar studies in several countries, in order to estimate induced abortion rates and the rates of women treated in health facilities for abortion-complications ${ }^{22,23,24}$.

The research consisted of a national data from health professionals and health facilities. The qualitative research explored the views of women in the context of seeking care for post-abortion complications: focus group discussions and in-depth interviews were conducted in 10 communities to gain insights from women who had recently had an abortion, and from men and health providers in their areas (details in chapter 7).

Thenational surveys assess the incidence of post-abortion complications and the availability andquality of post-abortion care (PAC) services in the country. Data collection for the quantitative study was carried out using two surveys:

- Health FacilitiesSurvey (HFS)

- Health ProfessionalsSurvey (HPS)

The Health FacilitiesSurvey was carried out at: teaching hospitals, District Headquarter Hospitals (DHQs), Tehsil Headquarter Hospitals (THQs), and Rural Health Centres (RHCs) in the public sector; and at their equivalently sizedhealth facilities in the private sector. In each facility, using a structured questionnaire, data were collected through direct interviews with health care providers working in the female or gynaecology/obstetrics department. The primary aim of this inquiry was to assess the capacity to provide quality post-abortion services and the number of women being treated for post-abortion complication nationally, regionally and by type of facility.

The Health ProfessionalsSurvey interviewed a range of health professionals, including gynaecologists, female doctors, LHVs/Nurses/Midwives, health managers, and researchers or policy makers dealing directly with (and therefore knowledgeable about) abortion and postabortion care. The HPS aimed to obtain the perceptions of health professionals regarding induced 
abortion and post-abortion complications, independently of the particular facility or organisation to which they were affiliated.

\section{1: Health Facilities Survey}

This quantitative survey was based on a sample of health facilities in four provinces of Pakistan. The sampling design involved several steps, and a great deal of attention was paid to develop the best possible sample design.

Step 1: Sampling of districts:Factors that were taken into account in selecting 24 of Pakistan's 114 districts across four provinces included: representation of the four provinces and poverty levels (see the ranking of districts according to poverty at the district level in Annex 1).The numbers of districts were selected in proportion to population size of each province. The districts were selected to represent a range across the poverty/socio economic rankings within each province as represented in Table A3. Districts were finalized based on the security situation at the time of the field work. The availability of comprehensive listings of private-sector facilities was a strong consideration. 24 districts were selected for inclusion in the final sample. They included 8 districts in Sindh, 10 in Punjab, 3 in Balochistan and 3 in KPK, and covered 35\% of Pakistan's total population. In order to includeall teaching hospitals approved by the Pakistan Medical and Dental Council (PMDC), 12 more districts (including Islamabad) were included, making the total of 36 study districts. The distribution of study districts is shown in Figure 2.1.Annex 2 shows the names of 36 districts along with their health statistics and social indicators. 
Figure 2.1: Geographical distribution of study districts

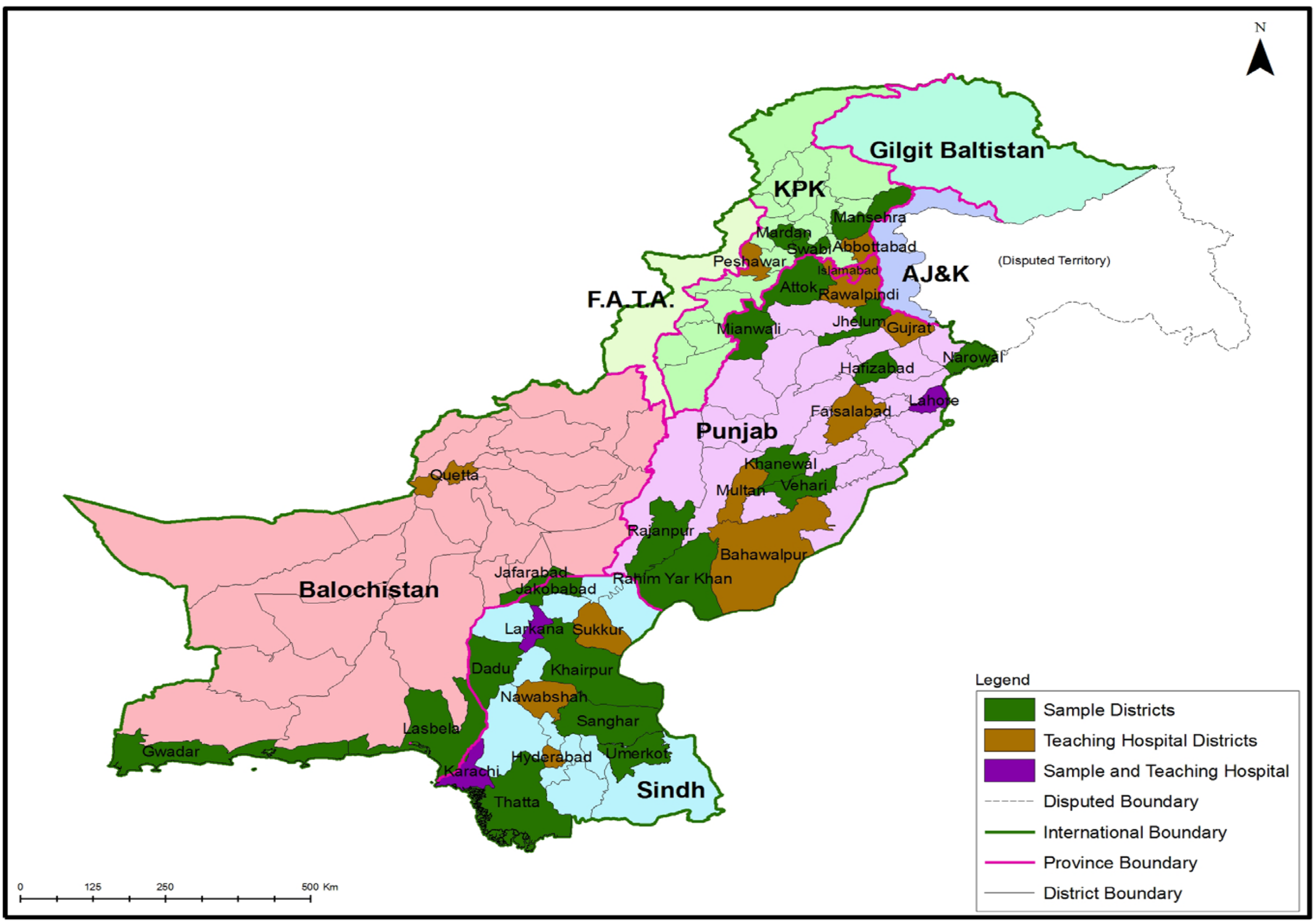


Step 2:Selection of thehealth facilities:From the 36 districts sampled, a sample of 266 facilities was selected, based on the assumption that this size would provide a reasonable coverage of all public and private facilities in Pakistan that treat women with post-abortion complications. The sample involved three types of health facilities:

(i) Teaching hospitals: We included all major teaching hospitals, public and private, that were recognized by the Pakistan Medical and Dental Council (PMDC) and offered gynaecology/obstetric services. For this purpose, a complete list of all the teaching hospitals was obtained from PMDC for the year 2011. Thirty-six public-sector teaching hospitals and 34 privatesector teaching hospitals in the four provinces fit these criteria. Interviews were successfully completed in 54 teaching facilities ( 33 public and 21 private). Interviews could not be conducted in thirteen private and three public teaching hospitals, as shown in Table 2.1.

Table 2.1: Selection of teaching hospitals by sector

\begin{tabular}{lrr} 
& Public & Private \\
Teaching Hospitals & 36 & 34 \\
Visited & 33 & 21 \\
Interview refused & 1 & 2 \\
Hospital not covered & 2 & 11 \\
\hline
\end{tabular}

Source: Pakistan Medical and Dental Council (PMDC), 2011.

Note: Teaching hospitals with gynaecology and obstetrics services and 50 or more beds were considered for selection. Private teaching hospitals were those attached to medical colleges established after 2008.

(ii) Public-sector non-teaching facilities: For the public sector, a stratified systematic random-sampling design was used to select a national representative sample of facilities. The two main factors for stratifying facilities were:

- Province (Punjab, Sindh, Balochistan and KPK)

- Type of facility (District Headquarter Hospitals (DHQs), Tehsil Headquarter Hospitals (THQs) and Rural Health Centres (RHCS).

Comprehensive listings of public-sector facilities were obtained from the HMIS (Health Management Information System) of the Provincial Departments of Health. Approximately $25 \%$ of DHQs, $27 \%$ of THQs and $11 \%$ of RHC's were sampled. These proportions were adequate to represent variation in each type of facility, and for scaling up to provide representative estimates for the universe of these facilities. A few of the sampled public facilities in Sindh province had been closed/damaged or were inoperative after the floods of 2010-2011. These facilities were 
replaced with facilities of a similar level, randomly selected from the original sampling list. In the final sample a total of 131 non-teaching facilities were selected-as shown in Table 2.2.

Table 2.2: Sampling of public-sector health facilities, by province

\begin{tabular}{|c|c|c|c|c|c|c|c|c|c|c|c|c|}
\hline \multirow[b]{2}{*}{ Province } & \multicolumn{3}{|c|}{ DHQs } & \multicolumn{3}{|c|}{ THQs } & \multicolumn{3}{|c|}{ RHCs } & \multicolumn{3}{|c|}{ Total } \\
\hline & $\begin{array}{l}\text { Universe } \\
\text { (HMIS) }\end{array}$ & Selected & $\begin{array}{l}\% \text { facilities } \\
\text { selected }\end{array}$ & $\begin{array}{l}\text { Universe } \\
\text { (HMIS) }\end{array}$ & Selected & $\begin{array}{c}\% \text { facilities } \\
\text { selected }\end{array}$ & $\begin{array}{l}\text { Universe } \\
\text { (HMIS) }\end{array}$ & Selected & $\begin{array}{c}\% \text { facilities } \\
\text { selected }\end{array}$ & $\begin{array}{l}\text { Universe } \\
\text { (HMIS) }\end{array}$ & Selected & $\begin{array}{c}\% \text { of } \\
\text { National }\end{array}$ \\
\hline Punjab & 35 & 10 & 29 & 85 & 20 & 24 & 284 & 30 & 11 & 404 & 60 & 15 \\
\hline Sindh & 18 & 8 & 44 & 46 & 15 & 33 & 111 & 17 & 15 & 175 & 40 & 23 \\
\hline KPK & 21 & 3 & 14 & 19 & 6 & 32 & 86 & 9 & 10 & 126 & 18 & 14 \\
\hline Balochistan & 23 & 3 & 13 & 15 & 3 & 20 & 89 & 7 & 8 & 127 & 13 & 10 \\
\hline Pakistan & 97 & 24 & 25 & 165 & 44 & 27 & 570 & 63 & 11 & 832 & 131 & 16 \\
\hline
\end{tabular}

Source: The Health Management Information System (HMIS) covers the provincial health departments of Pakistan in 2011.

(iii) Private-sector non-teaching facilities:In Pakistan no comprehensive list of private health facilities is available. However, in a study conducted during 2008-2010, the Population Council conducted a census to map public- and private-sector health facilities in 35 districts of Pakistan, using the Geographical Information System (GIS) ${ }^{25}$. Through this work, a comprehensive list of private sector facilities was made available for the 35 districts, making it possible to include a representative sample of these facilities in the 2012 HFS. Comprehensive listings based on a census of private-sector facilities were available for the 24sampled districts*, as discussed above. A stratified systematic random sampling design was used to select a representative sample of private-sector facilities, as was done for public facilities. The two main factors for stratifying private-sector health facilities were:

- Province (Punjab, Sindh, Balochistan and KPK) and

- Type of facility, using bed size to create categories that are equivalent to the three publicsector levels (Large hospitals with 81 or more beds were considered equivalent to publicsector DHQ hospitals; medium-sized hospitals with 20-80 beds were considered equivalent to public-sector THQ hospitals; and small hospitals with 5-19 beds were considered equivalent to RHCs.

\footnotetext{
* Four of the 24 sampled districts (Mianwali, Attock, Narowal and Hafizabad, all in Punjab province) did not have comprehensive listings of private-sector facilities through prior GIS mapping survey, so a rapid count and listing of private sector facilities was carried out for this study.
} 
Four large private facilities (equivalent to DHQs), 12 medium-size facilities (equivalent to THQs)and 65 small facilities (equivalent to RHCs) were selected in the sample. Originally these private facilities were selected using systematic random sampling. Lists of private health facilities within each sampled district were available through GIS mapping survey of Population Council. The health facilities were sorted by category i.e. Large, Medium and Small hospital based on bed size. According to the determined number of private health facilities of each category (Large, Medium and Small) within each province sample interval was calculated and the first facility was selected using a random number from random number table. However, once they were in the field, the research teams found that several private facilities that were visited were not in a position to provide PAC services. Private-sector facilities, shown in Table 2.3, are far more numerous than their equivalents in the public sector. So in the end a decision was taken to select only those facilities which had at least one female service provider available and where services related to gynaecology and obstetrics were being provided. Thus, the private-sector sample shown in Table 2.3 was based on a "reduced" census of facilities. Roughly $60-80 \%$ of privatesector facilities originally listed in the census were providing PAC and these became the basis of our final selection.

Table 2.3: Selection of private non-teachinghealth facilities, Health Facilities Survey, 2012

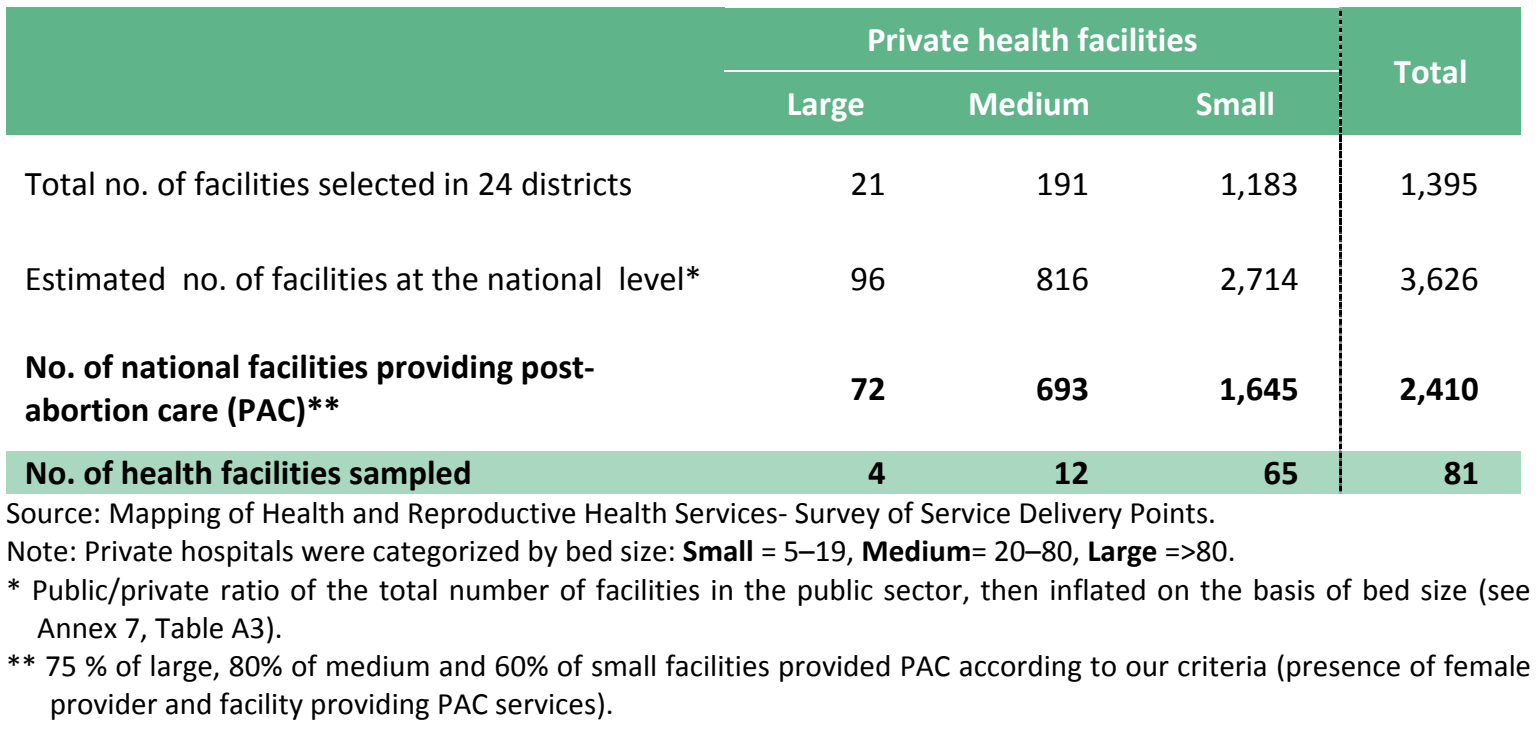

The final distribution of facilities included in the 2012 HFS is shown in Table 2.4. Generally there was better representation of larger facilities, particularly teaching hospitals and DHQs. However, there is a substantial sample (128) of smaller facilities (public RHCs and their equivalents in the private sector). The number of private sector facilities sampled was about two-thirds of the number of sampled public-sector facilities. Compared to the public sector, sample fractions were much smaller for the private sector, due to the much larger number of private sector facilities. 
Table 2.4: Number of health facilities included in the Health Facilities Survey, 2012

\begin{tabular}{|c|c|c|c|c|c|c|c|c|c|c|c|}
\hline \multicolumn{6}{|c|}{ Public sector } & \multicolumn{6}{|c|}{ Private sector } \\
\hline Facilities & Punjab & Sindh & KPK & Balochistan & Total & Facilities & Punjab & Sindh & KPK & Balochistan & Total \\
\hline $\begin{array}{l}\text { District } \\
\text { Headquarter } \\
\text { Hospitals (DHQs) }\end{array}$ & 10 & 8 & 3 & 3 & 24 & Large & 2 & 1 & 0 & 1 & 4 \\
\hline $\begin{array}{l}\text { Tehsil } \\
\text { Headquarter } \\
\text { Hospitals (THQs) }\end{array}$ & 20 & 15 & 6 & 3 & 44 & Medium & 6 & 4 & 1 & 1 & 12 \\
\hline $\begin{array}{l}\text { Rural Health } \\
\text { Centres (RHCs) }\end{array}$ & 30 & 17 & 9 & 7 & 63 & Small & 41 & 15 & 5 & 4 & 65 \\
\hline Total & 60 & 40 & 18 & 13 & 131 & Total & 49 & 20 & 6 & 6 & 81 \\
\hline $\begin{array}{l}\text { Teaching } \\
\text { hospitals }\end{array}$ & 17 & 9 & 5 & 2 & 33 & $\begin{array}{l}\text { Teaching } \\
\text { hospitals }\end{array}$ & 7 & 10 & 4 & 0 & 21 \\
\hline Total & 77 & 49 & 23 & 15 & 164 & Total & 56 & 30 & 10 & 6 & 102 \\
\hline
\end{tabular}

\section{2: Health Professionals Survey}

The aim of interviewing health professionals was to elicit information on their perceptions of all aspects of induced abortion and induced abortion complications, independent of the particular facility or organization to which they are attached. The target sampling size was above 100 health professionals across a wide range of professions including gynaecologists from teaching (category I) and non-teaching(category II) hospitals, doctors with gynaecological experience (category III), mid-level providers such as LHVs, Nurses and midwives(category IV)as well as number of nonmedical professionals such as researchers and policy makers (category $\mathrm{V}$ ). These respondents were specifically selected because of their reputed knowledge, understanding and interest in women's reproductive health in Pakistan.

The steering committee recommended that the majority of respondents be affiliated with the medical profession, especially gynaecologists/obstetricians as they were expected to have a better understanding of and greatest exposures to the post-abortion complications and would be a rich source of information. Consequently 75 percent of the total sample were medical doctors. The list of obstetricians and gynaecologists obtained from the Society of Obstetricians and Gynaecologists of Pakistan (SOGP) was used for sampling. The selection criteria included exposure to PAC and provincial representation. In Punjab, 36 health professionals were interviewed, 37 in Sindh, 17 in KPK, and 12 in Balochistan (Table 2.5). 
Table 2.5: Number and category of interviewed health professionals, by province, Health Professionals Survey, 2012

\begin{tabular}{lr|rrrr} 
& \multicolumn{5}{|c}{ Provincial Distribution } \\
Category & Total & Punjab & Sindh & KPK & Balochistan \\
Gynaecologists from public teaching hospitals & 16 & 5 & 5 & 3 & 3 \\
Gynaecologists from private teaching hospitals & 15 & 5 & 7 & 3 & 0 \\
Gynaecologists from non-teaching hospitals & 21 & 10 & 6 & 4 & 1 \\
Woman Medical Officer (WMO) & 24 & 8 & 8 & 4 & 4 \\
Lady Health Visitors, nurses, midwives & 18 & 5 & 8 & 2 & 3 \\
Health managers/researchers & 8 & 3 & 3 & 1 & 1 \\
Total (N) & 102 & 36 & $\mathbf{3 7}$ & $\mathbf{1 7}$ & $\mathbf{1 2}$
\end{tabular}

The characteristics of the health professionals interviewed for the survey are given below in Table

2.6.

Table 2.6: Characteristics of respondents, Health Professionals Survey, 2012

\begin{tabular}{|c|c|c|}
\hline & \multicolumn{2}{|c|}{2012} \\
\hline & $\%$ & $\mathbf{N}$ \\
\hline \multicolumn{3}{|l|}{ Province name } \\
\hline Punjab & 35 & 36 \\
\hline KPK & 17 & 17 \\
\hline Sindh & 36 & 37 \\
\hline Balochistan & 13 & 13 \\
\hline \multicolumn{3}{|l|}{ Gender of respondent } \\
\hline Male & 5 & 5 \\
\hline Female & 95 & 97 \\
\hline \multicolumn{3}{|l|}{ Current primary profession of respondent } \\
\hline Qualified gynaecologist & 51 & 52 \\
\hline Doctor & 24 & 24 \\
\hline Paramedical Staff & 18 & 18 \\
\hline Researcher /Policy maker/Health Managers & 8 & 8 \\
\hline \multicolumn{3}{|l|}{ Category of facility } \\
\hline Public & 73 & 74 \\
\hline Private & 27 & 27 \\
\hline \multicolumn{3}{|l|}{ Rural work experience for 6 month or more } \\
\hline Yes & 42 & 43 \\
\hline Total & 100 & 102 \\
\hline
\end{tabular}

Source: Health Professionals Survey.

\section{3: Technical Advisory Group}

During the preparatory phase of the study, an advisory group (whose members are listed in Annex 3)made up ofleading specialists on reproductive health in Pakistan, was formed to advise the team on study tools, provide technical guidance throughout the study period and ensure that protocols 
were being followed and that the study reached maximal coverage. The group also provided guidance in implementing the study and formulating recommendations. Two meetings were held: one before finalization of the study protocols; and the second to share the initial findings of the study and to obtain feedback for further analysis.

\section{4: Study tools/instruments}

The HPS and HFS questionnaires used during the 2002 study were the starting point for developing instruments for the current study. However, these tools were substantially revised to meet the expanded objectives and answer the study questions raised in the current study. As far as possible, comparability was maintained in the wording of questions to permit documentation of changesbetween 2002 and 2012. Draft tools and study protocols were presented to the Population Council's Institutional Review Board (IRB) for ethical approval. After incorporating the comments received from IRB, the revised protocols of the project were approved by the board. Questionnaires to be used in the Health Facilities Survey (HFS) and the Health Professionals Survey (HPS) were also reviewed by the project's Technical Advisory Group. Medical perspectiveswere obtained through discussions with the National Committee for Maternal \& Neonatal Health (NCMNH).

For the Health Facilities Survey (HFS), a structured questionnaire was used to conduct face-to-face interviews with the medical staff of sampled health facilitiesasking themabout women's postabortion complications and care. Respondents primarily included service providers in the female/gynaecology \& obstetrics department of the visited health facilities.Data were collected on service availability, specifically staffing, medical procedures and the availability of basic equipment for the care of post-abortion complications. A key purpose of this interview was to obtain respondents' estimate of the number of women treated for post-abortion care at their facility using two different reference periods: the estimated number of women treated at the facility in the past month, and the estimated number treated in an average month.

Information was also obtained on the provision of post-abortion contraceptive counselling and services, as well as the opinions and attitudes of service providers on a range of issues regarding the provision of post-abortion care. The additional topics covered in 2012 included information about providers trained in manual and electric vacuum aspiration, medication abortion and birth spacing/FP. Also added to the 2012 survey were questions on the availability of contraceptives at the facility, prescribed family planning methods and referral of clients for family planning, the availability of staff around the clock, suggestions for ways to reduce unsafe abortion and the availability of PAC equipment and medicines. 
For the Health Professionals Survey (HPS), a structured questionnaire was used to interview respondents and ask them forestimates of rural, urban, poor and non-poor women seeking abortion services, experiencing complications and receiving care for these complications. The questionnaire used in the 2002 study was revised and additional information was included, e.g., on the use of misoprostol for abortion and post-abortion care. Modules were added to cover topics such as the barriers that poor and rural women face in obtaining abortion and postabortion care. In addition, questions seeking the respondent's views on the opinions and attitudes of service providers toward contraception, abortion and post-abortion care and a separate module on post-abortion counselling were also added to the 2012 survey.

\section{5: Implementation}

Before visiting the study areas, to ensure smooth and efficient data collection, fieldwork plans were drawn up by each of the four provincial data collection teams. Efforts were made to improve the data collection process through prior correspondence with and permissions obtained from the relevant authorities.Visits were then made by the study coordinator/manager to build rapport with the concerned authorities and coordinate with key stakeholders to gain their support and assure them of the confidentiality of the data. While there was not much difficulty in securing the participation of health professionals at the majority of public-sector health facilities, getting private-sector providers to participate and provide information accurately proved to be challenging.

\section{6: Training, field work and data collection:}

All candidates shortlisted to carry out the data collection were interviewed by the study management teameither by telephone or directly before a final selectionwas made.Two types of team members were hired-medical doctors and social scientists (the data collection teams are listed in Annex 4).A 10-day training in research methods and tools was conducted in Islamabad in April, 2012. Data collection teams were briefed on a range of issues surrounding abortion and post-abortion care. Interviewing skills were strengthened through interactive sessions, role play and group work. As a part of the training, pre-testing and actual data collection were also carried out for two days at eight health facilities in the districts of Rawalpindi, Islamabad and Jhelum.Prior to the initiation of fieldwork, permission for data collection was obtained through correspondence with the offices of Provincial Health Secretaries and Director General Health Services of each of the four provinces. Approval for data collection from provincial and district health departments was also obtained. The health departments of all four provinces, district health offices and health facilities extended full cooperation during the data collection process. 
Data collection was started immediately after the training of interviewers and was completed within two months. Each provincial data collection team worked under the supervision of a team leader/supervisor. Each team was further divided into sub-teams while visiting the individual facilities. Interviews for both surveys (HPS and HFS) were carried out after informed consent was taken from the respondents. Informed consent was taken from all the participants, giving options; providers after reading the form or after listening to the information in the consent form, either signed themselves or verbally agreed in which case the note taker counter signed.

\section{7: Monitoring and quality assurance}

A number of measures were instituted to ensure the maintenance of high quality standards in both data collection and analysis. Roles and responsibilities of each team member were clearly defined. Each member was provided with a written set of responsibilities and standard quality checklists. During the fieldwork, team supervisors visited the study sites to ensure that all protocols were being followed. Supervisors thoroughly checked the filled questionnaires for the completeness and accuracy of the data collected. Interviewers were provided with regular feedback on their questionnaires to improve the quality of the data. The questionnaire also had a built-in mechanism to reduce inaccuracies in data entry. In addition, data was also double entered to avoid any mistakes. There was a 3 percent error rate of data inthe first phase; these errors were rectified by generating error listings and by consulting each of the questionnaires. The corrections were then made in final data entry file.

There were multiple risk factors involved in the fieldwork: the political and security situation in Balochistan and in KPK was particularly precarious and could suddenly change in ways that would prevent fieldwork in selected districts and areas of the study. In such cases, the Technical Advisory Group was especially helpful in identifying the areas with high security risk. However no untoward incident occurred during the field work and we did not need change the strategy except one RHC in Jaffarabad district was replaced due to security reasons.

\section{8: Data management and analysis}

Questionnaires were assigned serial numbers and checked for any quality issues when they were received from the field. Immediate feedback was given to the respective team in case of any discrepancy. Double data entry was done through CSPro, an industry standard package. After data cleaning and editing the two datasets were checked against original questionnaires and transcripts for inconsistency, and corrected as needed.

The analysis was done using SPSS Version 19. Consistency and range checks were done verify the quality of the data, and original data were recoded as needed to provide data for presentation in 
tables. Results presented are means, proportions, descriptive statistics, percent distributions, two and three-way cross-tabulation of relevant variables, and aggregate counts of events. No complex analytical techniques were used, as the report presents the descriptive findings.

\section{9: Limitations of the Study}

In Pakistan as elsewhere, getting direct information from women about abortion and postabortion complications is difficult because of the stigma and reluctance attached to the answers. Information if gathered from women, at least in a quantitative enquiry, is likely to be restricted if not impossible to collect and flawed. This study therefore relies on health professionals and on facility staff for responses to characteristics of women seeking abortion and post-abortion care and of women that present at their facilities. In particular, the Health Professionals selected for the Health Professional survey are highly knowledgeable experts in the field and their opinions/perceptions and their responses are found to be closely indicative of current realities. The methodological approach used in this study is based on an internationally accepted methodology developed by the Guttmacher Institute to approach the estimation of incidence of abortion and post-abortion complications worldwide. It is the same approach utilized in the earlier study carried out in Pakistan by the Population Council in 2002.

We have included a substantial sample of private sector facilities, in recognition of its expanding role in the delivery of abortion and other reproductive health care. However, a study limitation was the lack of full Census of Private health facilities. Fortunately we were able to use such a Census for all but four of the districts in our sample because of the GIS Census of health facilities conducted by the Population Council in 2008-9. These lists are three years old but were still comprehensive enough for the sampling of private sector facilities by bed strength. For the other four districts we collected fresh listings of private sector facilities. While we now have a comprehensive and reliable idea about the private sector's role in abortion related care, the fact that the 2002 study did not include the private sector, restricts some of our Health facility survey comparisons to the public sector, and limits our ability to compare all facilities over time.

While hospital statistics were also collected and could have been used as a cross check for the numbers provided by facility staff, this information was found to be patchy and incomplete. There was also a reluctance to share these data. The private sector is particularly weak in maintaining records on abortion related care. The Guttmacher methodology has tackled this problem in their world-wide approach by using the averages of the reported number of complication cases in the last month and the ones reported in an "average" month. This allows for variation in the calendar fluctuations and memory recall over a full year. 


\section{Chapter 3}

\section{The Practice and Health Consequences of Induced Abortion AmongWomen in Pakistan}

This chapter presents findings from the Health Professionals Survey (HPS) describing the characteristics of Pakistani women who seek abortions to terminateunwanted pregnancies, the various providers who offer these services, the methods used, and the financial costs of abortion care. We also look at the perceived risk of post-abortion complications by type of service provider. Patterns between the surveys conducted in 2002 and 2012 are compared.

\section{1: Profile of abortion clients}

Participants in the HPS were asked about the demographic characteristics of women who seek induced abortion. The groups they consider most likely to seek induced abortions are: married women ( $88 \%$ believed that most women having an abortion were married), women over 30 years of age (64\%), women with five or more children (65\%), uneducated (with no education or no formal education) women (70\%), poor women (77\%) and women living in rural areas (60\%). More than half (54\%) of the health professionals interviewed in 2012 were of the view that women seeking abortion are usually accompanied by relatives other than their husbands or mothers-inlaw. Slightly less than one-third reported that husbands usually accompanied their wives for this purpose, and the same proportion of respondents thought that a female friend usually accompanied the woman. One-quarter of the respondents thought that the woman would usually be accompanied by her mother-in-law, and 10\% thought that women seeking abortion services are usually unaccompanied (Table 3.1). 
Table 3.1: Characteristics of women most commonly perceived as seeking termination of unwanted pregnancy, Health Professionals Survey, 2002 and 2012

\begin{tabular}{|c|c|c|}
\hline & 2002 & 2012 \\
\hline Characteristics: & $\%$ & $\%$ \\
\hline \multicolumn{3}{|l|}{ Age group } \\
\hline $15-19$ & 4 & 5 \\
\hline $20-24$ & 8 & 9 \\
\hline $25-29$ & 21 & 23 \\
\hline $30-34$ & 40 & 38 \\
\hline $35-39$ & 20 & 21 \\
\hline 40 or more & 7 & 5 \\
\hline \multicolumn{3}{|l|}{ Marital status } \\
\hline Married & 96 & 88 \\
\hline Single & 4 & 12 \\
\hline \multicolumn{3}{|l|}{ Education } \\
\hline No education & 63 & 65 \\
\hline No formal education & 9 & 5 \\
\hline Primary or less & 3 & 9 \\
\hline Middle or higher & 26 & 21 \\
\hline \multicolumn{3}{|l|}{ No. of children } \\
\hline Nulliparous & 5 & 8 \\
\hline 1 to 2 & 5 & 4 \\
\hline 3 to 4 & 23 & 23 \\
\hline 5 or more & 68 & 65 \\
\hline \multicolumn{3}{|l|}{ Residence* } \\
\hline Urban & - & 40 \\
\hline Rural & - & 60 \\
\hline \multicolumn{3}{|l|}{ Economic status* } \\
\hline Poor & - & 77 \\
\hline Non-poor & - & 23 \\
\hline Total & 100 & 100 \\
\hline \multicolumn{3}{|l|}{ **Usually accompanied* } \\
\hline Husband & - & 31 \\
\hline Mother-in-law & - & 26 \\
\hline Sister & - & 16 \\
\hline Relatives & - & 54 \\
\hline Friends & - & 30 \\
\hline Alone & - & 10 \\
\hline Mother & - & 7 \\
\hline Others & - & 3 \\
\hline Total & (154) & (102) \\
\hline
\end{tabular}

Source: Health Professionals Survey.

*This information was not collected in the 2002 Health Professionals Survey.

**Multiple responses.

Overall these characteristics are similar to those reported a decade ago. However, certain changes in the overall profile can be observed bycomparing the 2002 and the 2012 studies.A slightly larger percentage of health professionals in 2012 than in 2002 observed that the group 
likely to be seeking abortion services would be the unmarried-an increase from $4 \%$ to $12 \%$. And the proportion reporting that the most common group of abortion seekers would be women with no children rose from 5\% in 2002 to almost $8 \%$ in 2012.

\section{2: Methods used toinduce abortion}

In the HPS of both 2002 and 2012, participants were asked about the type of methods that are used to terminate an unwanted pregnancy, by both service providers and women themselves. These questions were first asked unprompted and subsequently with prompting. The second part of this query asked about the two most common methods among those mentioned by respondents. We show both prompted and unprompted responses; however, only unprompted responses are used to show changesin the use of various abortion methods between 2002 and 2012.

\section{Methods used by abortion providers}

Without prompting,69\% of health professionals in 2012 reported that dilatation and curettage (D\&C) was a method commonly used by abortion providers, $68 \%$ mentioned misoprostol administered orally, and $41 \%$ mentioned misoprostol administered vaginally (Table 3.2). Surgical methods such as D\&E were cited by $31 \%$ of respondents, MVA by $26 \%$ and EVA by $7 \%$. However, with prompting, higher proportions of respondents cited these methods. The predominance of D\&C used to induce abortion is consistent with the 2002 survey, in which $72 \%$ of respondents mentioned this method. In the 2002 survey a large proportion of respondents also reported the use of methods such as laminariasticks (61\%), the IUCD (44\%) and anti-malarial drugs (33\%) for pregnancy termination. The proportions reporting these methods had declined considerably by 2012 , to $16 \%, 28 \%$ and $3 \%$, respectively, indicating a possible decline in the use of these more invasive and unsafe methods. 
Table 3.2: Methods used by service providers to terminate an unwanted/unplanned pregnancy, as perceived by respondents, Health Professionals Survey, 2002 and 2012

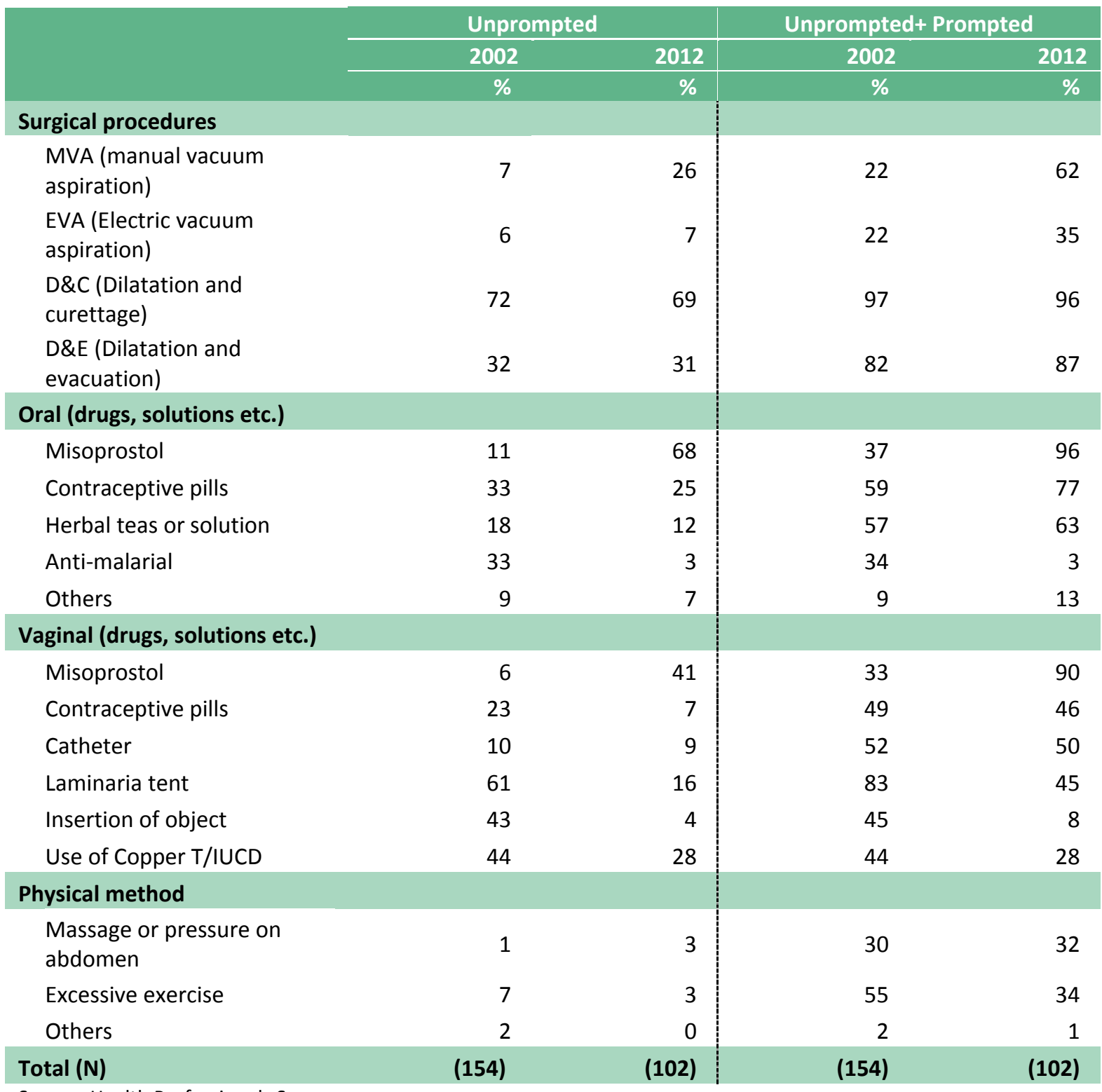

Source: Health Professionals Survey.

Note: Based on multiple responses.

Safe and non-invasive MVA/EVA techniques were cited by only $13 \%$ of health professionals in 2002 , but this proportion increased to $33 \%$ by 2012 . The most striking change is the increase in the reporting of misoprostol use for induced abortion. In 2002 very few health professionals mentioned misoprostol use for this purpose, whereas in 2012 more than two-thirds reported its oral administration and four in 10 mentioned itsadministration vaginally. 
In the 2002 survey, health professionals were asked about the two most common methods used by abortion providers to terminate a pregnancy. Three-quarters said $D \& C$ was the most common method, and more than one-quarter (28\%) ranked the laminariastick as the second most common method (Figure 3.1). In 2012 the same question was posed, but respondents were asked to differentiate between method use in urban and rural areas. For urban areas, more than one-third (36\%) of the health professionals ranked misoprostol as one of the two most common methods used to induce abortion, and one-quarter said D\&C was the second most common method. For rural areas*, about one in four respondents (24\%) ranked D\&C, and just over one in five (22\%) mentioned misoprostol as the two most common abortion methods. These findings suggesta decline in the use of D\&C between 2002 and 2012 andthe increasing emergence ofmisoprostol as a way of ending unwanted pregnancies.

Figure 3.1: The two most common methods used to terminate an unwanted/unplanned pregnancy as perceived by respondents, Health Professionals Survey, 2002 and 2012

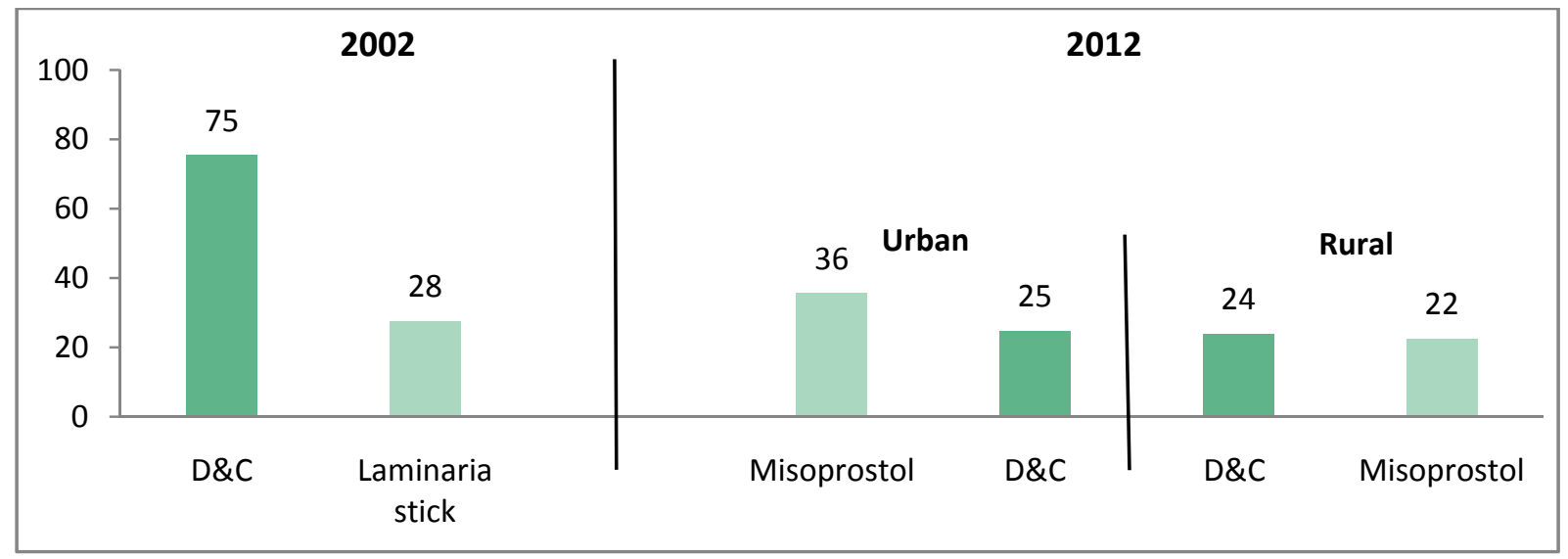

Source: Health Professionals Survey.

\footnotetext{
* Overall $42 \%$ of the health professionals had had 6 months or above direct experience of working in rural areas. HPs working in secondary and tertiary care facilities mostly treat patients coming from rural areas so quite knowledgeable about rural patients.
} 


\section{Methods used by women themselves}

Respondents were asked which abortion methods women were likely to use on their own. The reported use of drugs (quinine, ergot alkaloids) and of invasive methods (such asobjectsinserted into the vagina)was lower in 2012 than in 2002 (Table 3.3). And while misoprostol wasmentioned byfew respondents in the 2002 study, 44\% of the health professionals in the 2012 study said they thought women themselves were usingmisoprostol terminate an unwanted pregnancy. This finding suggests an increase in the useof safer methods by women bringing about their own pregnancy terminations, and a reduction in the use of methods likely to lead tohealth complications.

Table 3.3: Methods most commonly used by women who perform their own abortions as perceived by respondents, Health Professionals Survey, 2002 and 2012

\begin{tabular}{|c|c|c|c|c|}
\hline & \multicolumn{2}{|c|}{ Unprompted } & \multicolumn{2}{|c|}{ Unprompted+ Prompted } \\
\hline & 2002 & 2012 & 2002 & 2012 \\
\hline & $\%$ & $\%$ & $\%$ & $\%$ \\
\hline \multicolumn{5}{|l|}{ Drugs } \\
\hline Purgatives & 6 & 12 & 38 & 47 \\
\hline Quinine & 38 & 28 & 60 & 65 \\
\hline Ergot alkaloids & 17 & 9 & 47 & 52 \\
\hline Misoprostol & 9 & 44 & 9 & 80 \\
\hline Contraceptive pills, Gynaecosid & 49 & 37 & 50 & 83 \\
\hline \multicolumn{5}{|l|}{ Herbs } \\
\hline Oral & 24 & 42 & 64 & 84 \\
\hline Vaginal & 16 & 28 & 56 & 68 \\
\hline \multicolumn{5}{|l|}{ Other methods } \\
\hline Insertion of object into vagina & 34 & 28 & 61 & 67 \\
\hline Heavy exercise & 36 & 21 & 67 & 57 \\
\hline Heavy massage to abdomen & 12 & 9 & 38 & 41 \\
\hline Total (N) & (154) & $(102)$ & (154) & (102) \\
\hline
\end{tabular}

Source: Health Professionals Survey.

Note: Based on multiple responses.

Health professionals in 2002 were of the opinion that the two most common abortion methods used by women themselves were excessive exercise (67\%) and the ingestion of herbs (64\%). In 2012, 39\% of health professionals thought that for urban women misoprostol was the most common method, and $22 \%$ that contraceptive pills/Gynaecosidwas the second most common method (Figure 3.2). 29\% of respondents consideredthe ingestion of oral herbs to be the most common method used by rural women in 2012,and 19\% thought it was theinsertion of foreign objects into the vagina. This pattern (similar to the one found for methods use in general) also 
points towards an increasing use of less invasive abortion methods by women, especially in urban areas, compared to the situation in 2002. Nevertheless, the use of unsafe techniques still appears to be quite common in 2012 , especially in rural areas.

Figure 3.2: The two methods most commonly used by women who perform their own abortions as perceived by respondents, Health Professionals Survey, 2002 and 2012

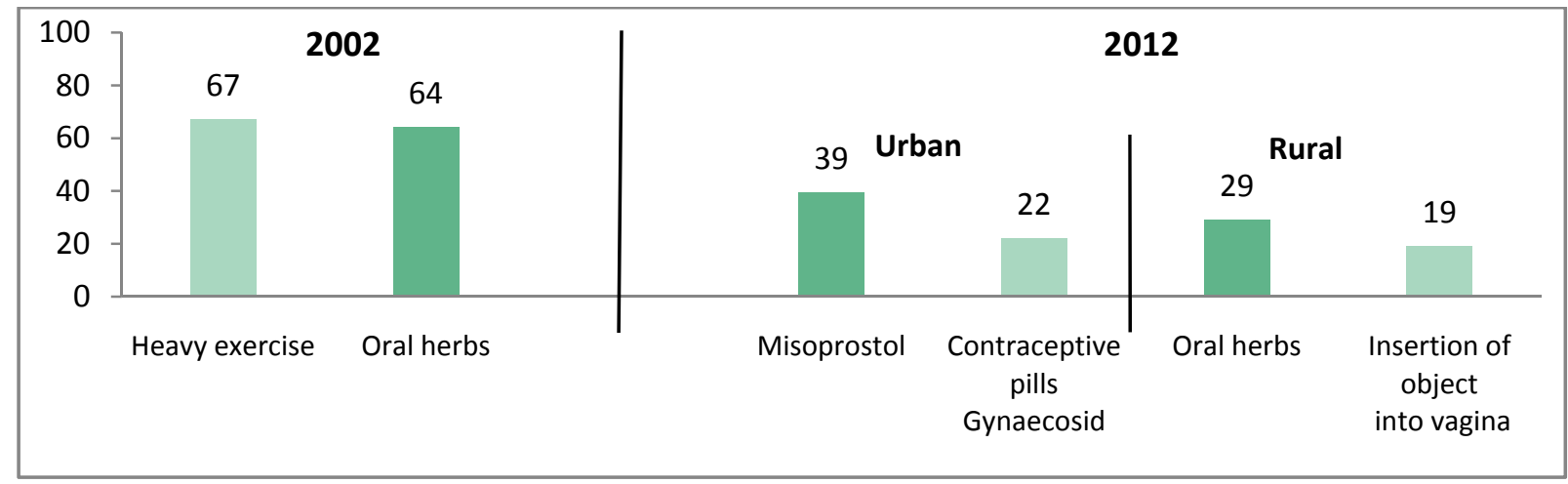

Source: Health Professionals Survey.

\section{3: Costs of induced abortion}

The cost of abortion generally varies with the skills of the provider, the duration of pregnancy and whether the client is married or unmarried (Population Council, 2004). Health professionals were asked what they perceived to be the average cost of a first-trimester abortion obtained from different types of providers in rural and urban areas and charged topoor and non-poor ${ }^{\ddagger}$ women.

The health professionals reported that in 2012 poor urban women were being charged Rs.7,000, on average, by private doctors, Rs.4,000 by LHVs/Nurses/Midwives/FHTs and Rs.2,400 by TBAs (Table 3.4). Urban non-poor women are thought to be payinghigher charges for the same providers: Rs.11,000for the services of a private doctor, Rs.6,000 to LHVs/Nurses/Midwives/FHTs and Rs.3,000 to TBAs. The costs for rural women are perceived to be lower than for urban women: poor rural women are believed to pay an average of Rs.5,000 to private doctors, Rs.3,000 to LHVs/Nurses/Midwives/FHTs and Rs.2,000 to TBAs. For non-poor ruralwomen, private doctors are also the most costly (Rs.7,000), followed by LHVs/Nurses/Midwives/FHTs (Rs.4,000) and TBAs $(\mathrm{Rs} .3,000)$. The cost is perceived to be lowest if women in any of the four categories of residence and poverty obtain abortion services from pharmacists/drug store.

\footnotetext{
"The classification of "poor and non-poor" and "rural and urban" was based on the perception of the provider who was being interviewed.
} 
Table 3.4: Mean and median cost* of a first-trimester abortion, by type of provider, according to residence and economic status of women as perceived by respondents, Health Professionals Survey, 2012

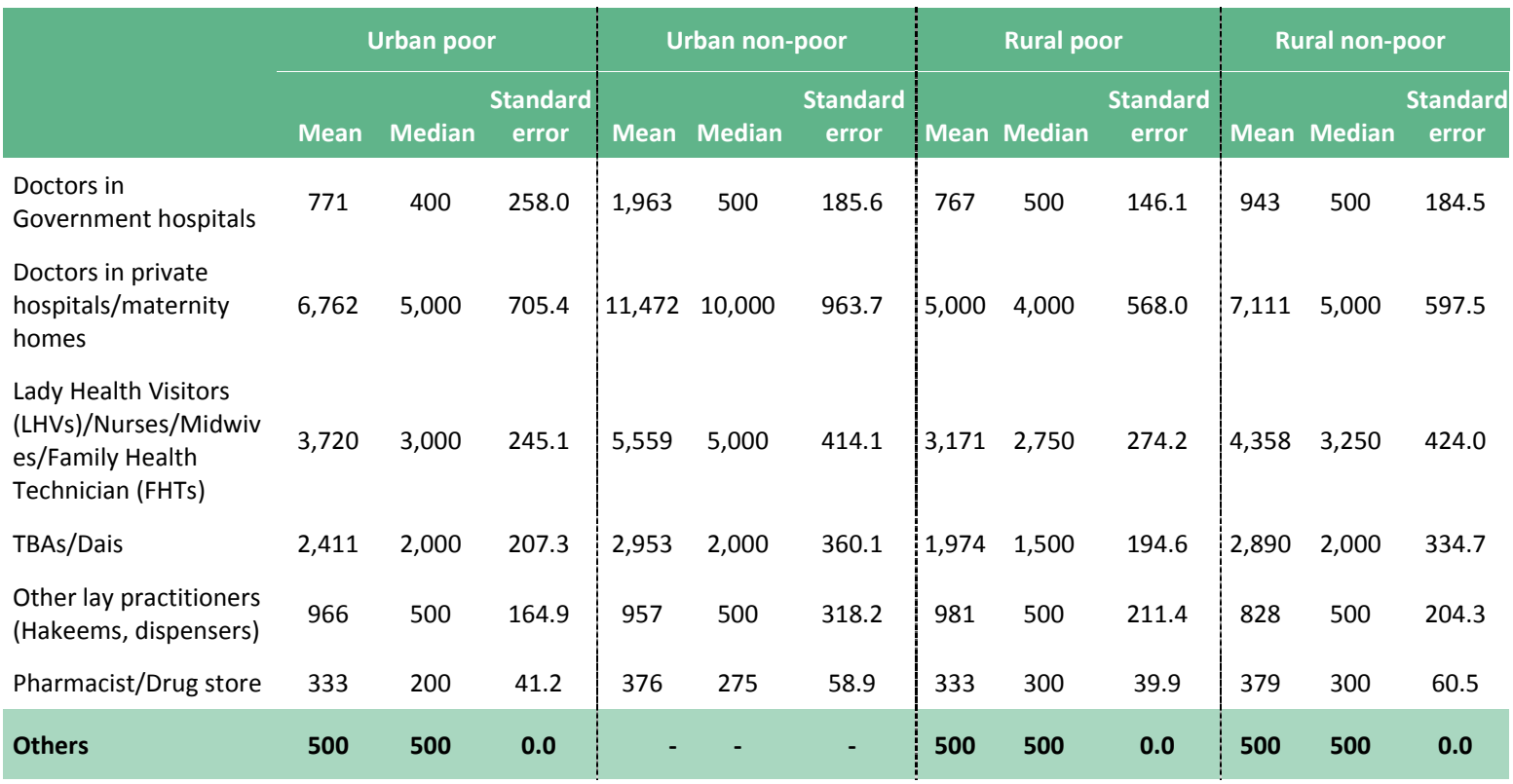

Source: Health Professionals Survey.

*Cost in Rupees, 2012.

Doctors working in private clinics are reported to charge about three times more than TBA/Dai and about twice as much as LHVs/Nurses/Midwives(Figure 3.3). Charges are also higher in urban than rural areas, and poor women, understandably, are charged less than non-poor women.

Figure 3.3: Average cost* of a first-trimester abortion, by type of provideras perceived by respondents, Health Professionals Survey, 2002 and 2012

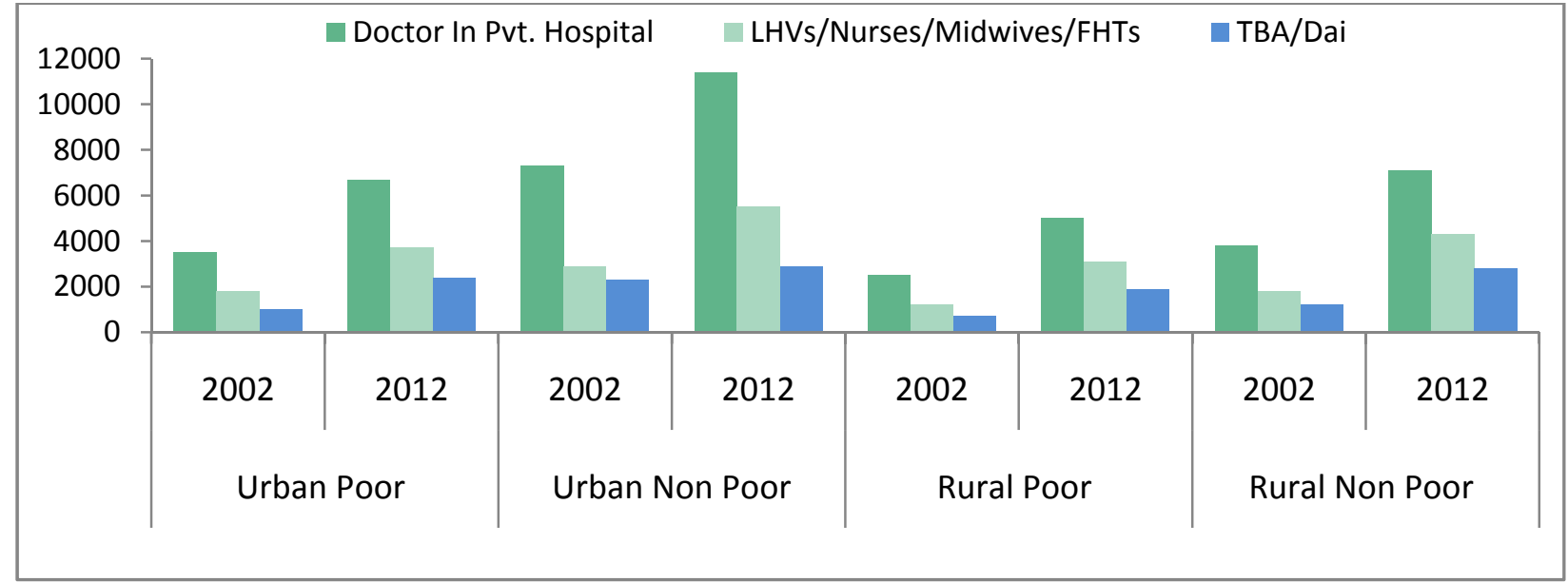

Source: Health Professionals Survey.

*Cost in Rupees, 2002 and 2012. 
The cost of induced abortion has nearly doubled in past 10 years. After accounting for inflation ${ }^{26}$ based on the Federal Bureau of Statistics /PBS data on inflation, we find costs have hardly changed across the two time periods (2002 and 2012). The estimated cost for both urban and rural poor women turning to LHVs, nurses and TBAs/Dais has seen the greatest relative increase (see Figure 3.3). Since these types of providers are the most common choice for poor women, theirability to payfor an abortion appears to have worsened. Regardless of their economic status, urban women have to pay more for an abortion than rural women,in both 2002 and 2012.

\section{4: Abortion providers and the risk of complications:}

In both 2002 and 2012, as Figure 3.4 and Figure 3.5 indicate, health professionals were asked whichtype of abortion providers were being used by four subgroups: poor urban, non-poor urban, poor rural and non-poor rural women. In 2012, they estimated that, around $40 \%$ of abortions obtained by poorurban women were performed by LHVs/Nurses/Midwives/FMTs (down from $45 \%$ in 2002$), 30 \%$ (34\% in 2002) by TBAs/Dais/lay practitioners, and $21 \%$ by doctors and gynaecologist (10\% in 2002)More than half of abortions obtained in 2012by non-poor urban women were believed to be performed by doctors and gynaecologists $(33 \%$ and $21 \%$ respectively), up from $49 \%$ in 2002 , and $27 \%$ by LHVs/Nurses/Midwives/FMTs, down slightly from $34 \%$ in 2002 . By 2012 , only $11 \%$ of abortions among non-poor urban women were believed to have been carried out byTBAs/Dais/lay practitioners. 
Figure 3.4: The type of provider used by urban women for obtaining abortions, by the women's economic status as perceived by respondents, Health Professionals Survey, 2002 and 2012
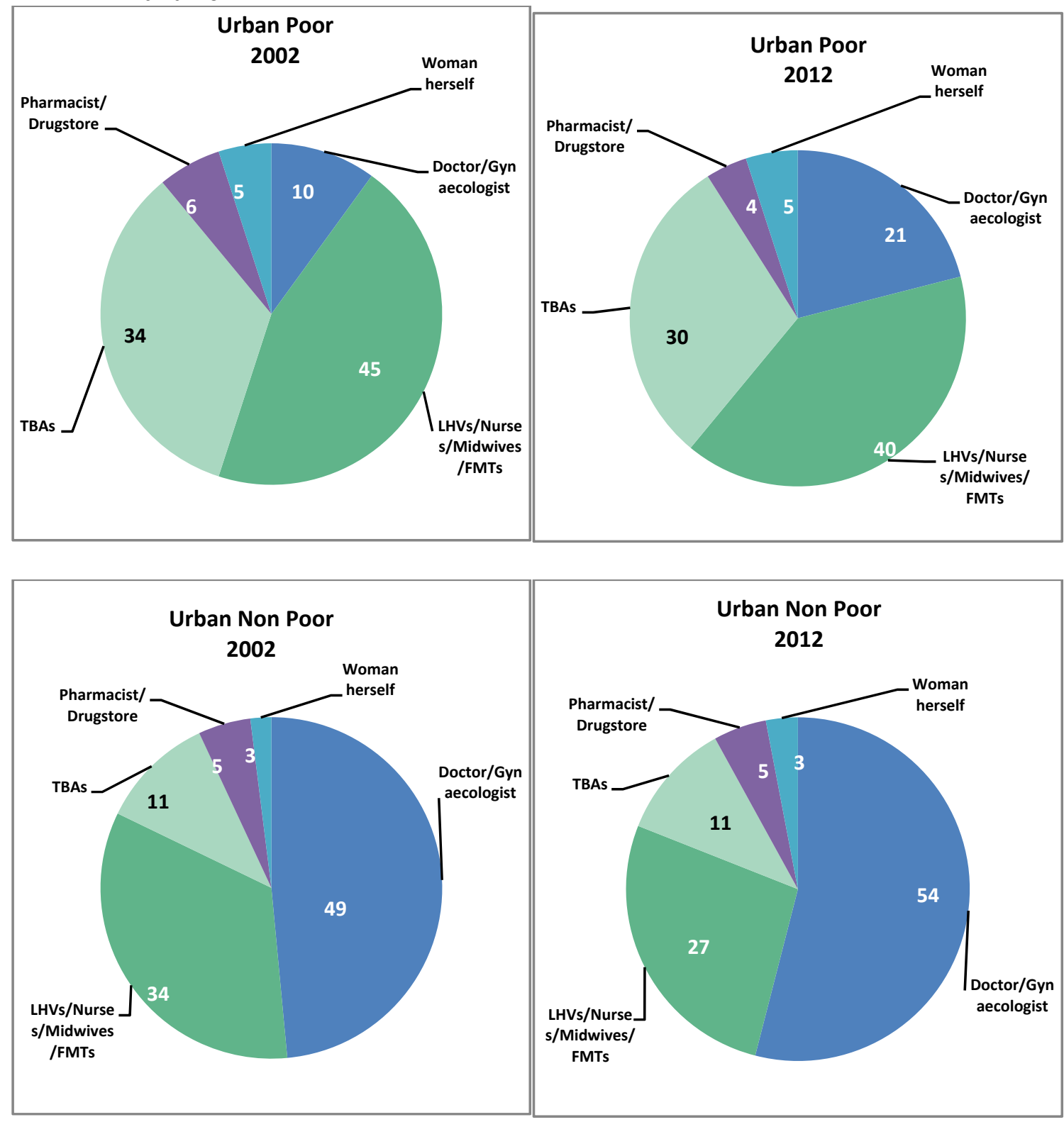

Source: Health Professionals Survey. 
Figure 3.5: The type of provider used by rural women for obtaining abortions, by the women's economic status as perceived by respondents, Health Professionals Survey, 2002 and 2012
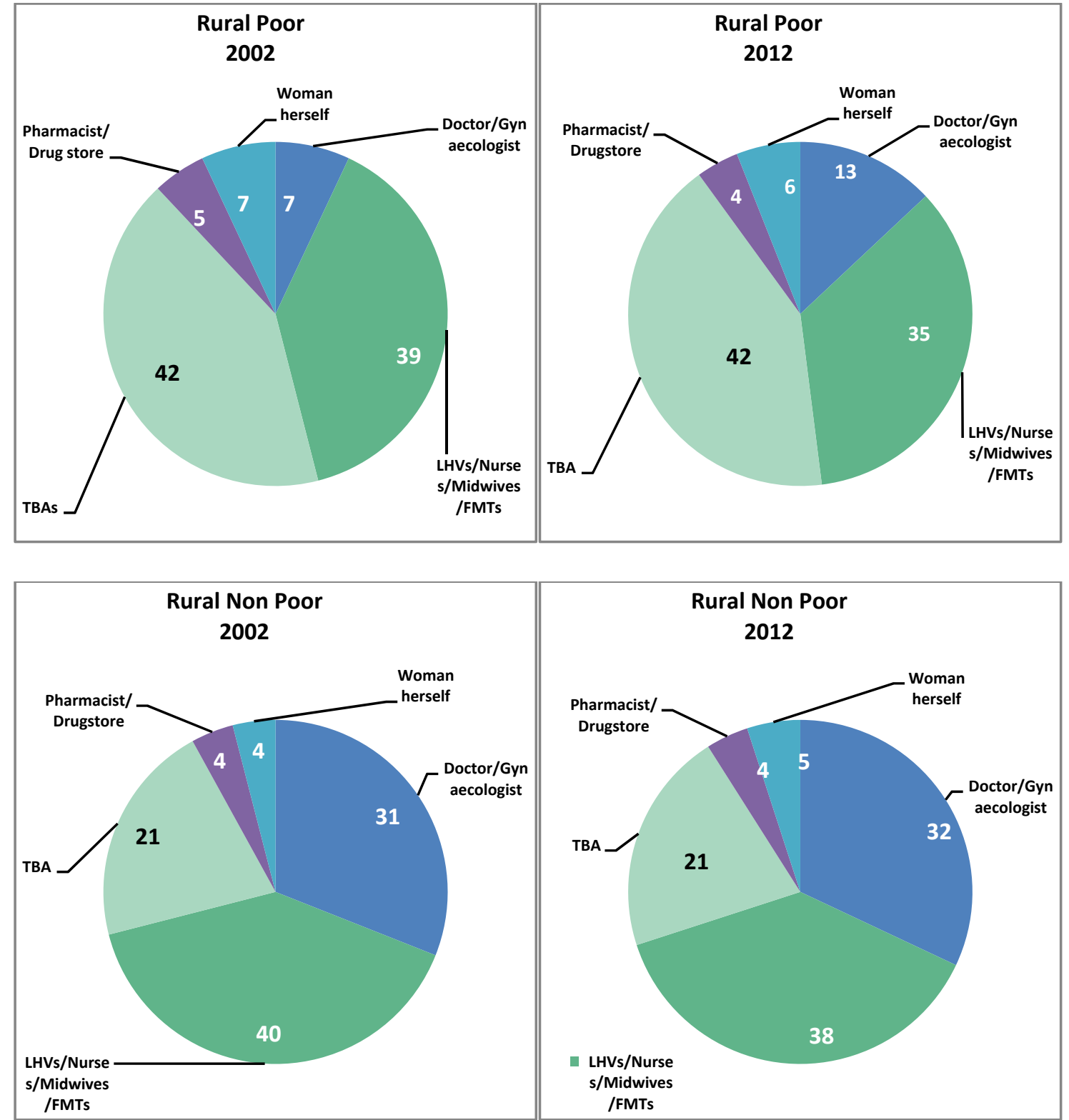

Rural Non Poor

2012

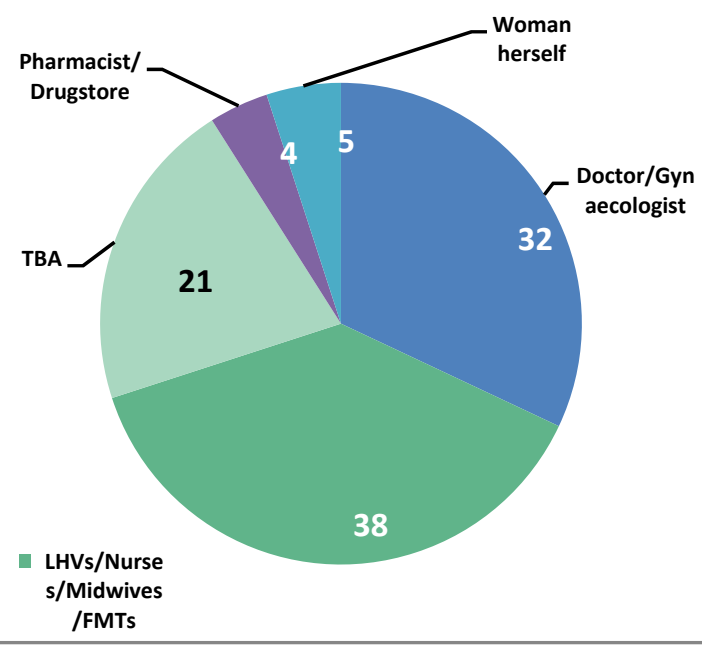

Source: Health Professionals Survey. 
For poor rural women, TBAs/Dais/lay practitionerswere thought to be the largest cadre of providers in 2012, as they were in 2002 (42\% in both years). While there has been a small decline in the proportion of abortions among this subgroup that are performed by LHVs/Nurses/Midwives/FMTs (from 39\% in 2002 to 35\% in 2012), the proportion of abortions performed by doctors is believed to have increased (from 7\% to 13\%). The least amount of change over the past 10 years was observed in the type of abortion providers used bynon-poorrural women: in 2012, one-third of abortions were reportedly performed by doctors/gynaecologists, almost four in 10 byLHVs/Nurses/Midwives/FMTs and one-fifth by TBAs/Dais/lay practitioners.

Comparison of the HPS findings for 2002 and 2012 suggests that in urban areas, both poor and non-poor women seeking an abortion are increasingly using the services of doctors. However, it appears that most abortions are still performed by mid-level providers- LHVs/Nurses/Midwives and that the proportion carried outby TBAs/Dais, bypharmacists/drug stores, or by women themselves has changed very little.

\section{The risk of complications varies with the type of provider}

According to the health professionals, regardless of whether women live in urban or rural areas and in both 2002 and 2012, health complications requiring medical treatment are most likely to occur when abortions are performed by TBAs/Dais.In 2012, the proportion of women expected to experience a complication from procedures performed by TBAs/Dais ranges from $55 \%$ among non-poor urban women to 68\% among poor rural women.In both 2002 and 2012, among all four subgroups of women, 4-5 out of 10 women were estimated to develop complications when an abortion is carried out by LHVs/Nurses/Midwives, and among one out of 10 women if the service provider was a gynaecologist (Table 3.5). 
Table 3.5: Proportion of women having abortions likely to experience complications, by type of abortion provider, according to women's residence and economic status, as perceived by respondents, Health Professionals Survey, 2002 and2012

\begin{tabular}{|c|c|c|c|c|c|c|c|c|c|c|c|c|c|c|c|c|}
\hline \multirow{3}{*}{$\begin{array}{l}\text { Type of } \\
\text { providers }\end{array}$} & \multicolumn{4}{|c|}{ Urban Poor } & \multicolumn{4}{|c|}{ Urban Non Poor } & \multicolumn{4}{|c|}{ Rural Poor } & \multicolumn{4}{|c|}{ Rural Non Poor } \\
\hline & \multicolumn{2}{|c|}{2002} & \multicolumn{2}{|c|}{2012} & \multicolumn{2}{|c|}{2002} & \multicolumn{2}{|c|}{2012} & \multicolumn{2}{|c|}{2002} & \multicolumn{2}{|c|}{ 2012: } & \multicolumn{2}{|c|}{2002} & \multicolumn{2}{|c|}{2012} \\
\hline & $\%$ & $n$ & $\%$ & $n !$ & $\%$ & $n$ & $\%$ & $n$ & $\%$ & $n$ & $\%$ ! & $n$ & $\%$ & $n$ & $\%$ & $n$ \\
\hline Gynaecologists & 10 & 128 & 9 & 100 & 7 & 138 & 10 & 100 & 13 & 99 & 9 & 95 & 10 & 119 & 10 & 97 \\
\hline WMOs (MBBS) & 25 & 139 & 23 & 100 & 21 & 141 & 23 & $100 !$ & 27 & 123 & 26 & 96 & 22 & 134 & 26 & 97 \\
\hline $\begin{array}{l}\text { LHVs/Nurses/ } \\
\text { Midwives } \\
\text { /FMTs }\end{array}$ & 48 & 148 & 45 & 100 & 42 & 140 & 41 & 100 & 50 & 139 & 49 & 98 & 46 & 136 & 45 & 98 \\
\hline TBAs/Dais & 66 & 143 & 64 & 100 & 61 & 120 & 55 & 100 & 67 & 134 & 68 & 98 & 65 & 117 & 58 & 97 \\
\hline $\begin{array}{l}\text { Lay } \\
\text { practitioners } \\
\text { (Hakeem, } \\
\text { dispenser) }\end{array}$ & 61 & 107 & 42 & 94: & 59 & 85 & 36 & 94 & 59 & 101 & 46 & 88 & 57 & 86 & 40 & 88 \\
\hline $\begin{array}{l}\text { Pharmacists/ } \\
\text { drug store }\end{array}$ & 30 & 71 & 36 & $95:$ & 27 & 68 & 31 & 95 & 30 & 69 & 39 & $88 i$ & 27 & 67 & 35 & 88 \\
\hline Women herself & 54 & 111 & 55 & 100 & 51 & 89 & 48 & $100 !$ & 57 & 99 & 54 & 97 & 54 & 82 & 47 & 96 \\
\hline $\begin{array}{l}\text { Number of } \\
\text { respondents }\end{array}$ & & (154) & & (102) & & (154) & & (102) & & (154) & & 02)! & & (154) & & (102) \\
\hline
\end{tabular}

Source: Health Professionals Survey.

For all types of abortion providers except pharmacists/drug stores, the risk of complications is believed to have changed very littlebetween2002 and 2012. However, health professionals perceive a notable decrease in the risk of complications for abortions performed by lay practitioners (Hakeems and dispensers).This may be explained by their increased use of safe drugs like misoprostol and their reduced use ofmore invasive methods. The probability of complications resulting from abortions carried out with the help of pharmacists/drugstores is thought to have increased among poor urban women (from $30 \%$ to $36 \%$ ) and among poor rural women (from $30 \%$ to $39 \%$ ). It is possible that pharmacists/drug stores are now providing misoprostol, in addition to other products that they provided in the past (i.e., contraceptive pills, Gynaecosid, quinine, purgatives and ergot alkaloids), and this could account for the somewhat increased risk of complications among women seeking abortion services from this source: For example, women may be given incorrect or insufficient information and they may use the method incorrectly. However, we have seen a substantial increase in the use of misoprostol by women themselves (from 9\% to 44\%), as shown in Table 3.3. When drugs like misoprostol are obtained from drug stores, women often use them without knowing how to use them correctly, and without being advised on their proper use (as it is the husbands who usually purchase these 
medicines from drug stores). Although misoprostol is considered a safe method when used under the supervision of a trained service provider, when it is self-administered a woman may have to make several visits to a health care provider to assess the effectiveness of the treatment, seek help for the management of withdrawal bleeding or side effects (nausea, vomiting, chills, fever, diarrhoea and headaches) and, in cases of incomplete abortion, need surgical intervention ${ }^{27}$. It is possible; therefore, that the health professionals took into consideration the overall discomfort and complaints they had observed fromwomen taking misoprostol at home and assumed that there would be a similar risk of post-abortion complications if the service provider was a pharmacist/drug store. 


\section{Chapter 4 Post-Abortion Care Patients and the Services they Receive}

\section{1: Introduction:}

Pakistan has a well-developed infrastructure of public health facilities. Healthcare is provided through a three-tiered healthcare delivery system. Basic Health Units (BHUs) and Rural Health Centres (RHCs) form the core of the primary healthcare model; secondary care, including first and second referral facilities, provide acute, ambulatory and inpatient care through Tehsil Headquarter Hospitals (THQs) and District Headquarter Hospitals (DHQs); and tertiary care is made up of teaching hospitals ${ }^{28}$. In recent years there has been a wide-scale expansion of health care services in the private sector ${ }^{28}$, which is a strong reason to study the role of the private sector in providing post-abortion care services and to compare its record to that of the public sector.

In the principles defined by the International Conference on Population and Development (ICPD) at Cairo in 1994, a ground-breaking consensus, ICPD called for all women to be given access to treatment for abortion-related complications, post-abortion counselling, and education and family planning services, regardless of the legal status of abortion in the country ${ }^{29}$. Being a signatory of ICPD, Pakistan undertook a series of actions to reflect the Cairo agenda. In 1999, the Reproductive Health (RH) Service Package was developed with the joint efforts of the Ministries of Health and Population Welfare. It provides broad guidelines outlining eight key components of necessary reproductive health care services. These include pre- and post-abortion care and comprehensive family planning, along with other components ${ }^{30}$. In 2000, a draft RH Policy was also presented, based on the ICPD's Plan of Action, but thiswas never approved by the Ministry of Health or the Ministry of Population Welfare ${ }^{31}$.

Post-abortion care (PAC) is a package of recommended health care services that should be available toall women who experience complications following a spontaneous or induced abortion $^{32}$. Complications from spontaneous abortions and unsafe induced abortions pose a serious global threat to women's health and lives. The World Health Organization (WHO) estimates that $10 \%-50 \%$ of women who have an unsafe abortion need medical care; some women who experience spontaneous abortions also need treatment. While most health systems provide treatment for abortion complications as part of emergency obstetric care, the infrastructure to 
make these services widely available usually is lacking in developing countries. The term "postabortion care" was first articulated as a critical element of women's health initiatives in a 1991IPAS strategic planning document, which encouraged "the integration of post-abortion care and family planning services in health care systems" as a means of breaking the cycle of repeat unwanted pregnancy and improving the overall health status of women in the developing world ${ }^{33}$.

\section{Essential elements of the PAC model}

The Essential Elements of thePAC model, endorsed by the PAC Consortium in May 2002 reflect from both a provider and a consumer perspective, an enhanced vision of high-quality, sustainable services. The model's five elements (see box below) shift the focus from facility-based medical treatment to a public health approach that responds to women's broader sexual and reproductive health needs.

\section{Essential Elements of Post-Abortion Care}

\section{Community and service provider partnerships}

- Prevent unwanted pregnancies and unsafe abortion

- Mobilize resources to help women receive appropriate and timely care for complications of abortion

- Ensure that health services reflect and meet community expectations and needs

\section{Counselling}

- Identify and respond to women's emotional and physical health needs and other concerns

\section{Treatment}

- Treat incomplete and unsafe abortion and potentially life-threatening complications

\section{Family planning and contraceptive services}

- Help women practice birth spacing or prevent an unwanted pregnancy

\section{Reproductive and other health services}

- Preferably provide on-site, or via referrals to other accessible facilities in provider's network

Source: Post-abortion Care Consortium Community Task Force, Essential Elements of Post-Abortion Care: an expanded and updated model, PAC in Action, 2002, No. 2, Special Supplement.

Incomplete abortion, failed abortion, haemorrhage, infection and uterine perforation are the major post-abortion complications. For the management of these complications, according to the fifth element of the PAC model ${ }^{34}$, theWHO recommends the following measures and procedures; 
1. Administration of misoprostol

2. Vacuum aspiration

3. Local and general anaesthesia

4. Dilatation and evacuation (D\&E)

5. Administration of drugs to stop bleeding

6. Administration of antibiotics for infection

7. Intravenous fluid replacement, blood transfusions

8. Laparoscopy or laparotomy

The present study was aimed to assess the readiness of the services in both the public and private health sectors to provide post-abortion care for spontaneous and induced abortions. We were particularly interested to explore some of the essential quality-of-care systems in place at the health facilities we surveyed. Although in the 2002 study, both public and private facilities were visited, the main focus was on the public sector. However, given the rapid expansion of the private health sector during the last decade,in the 2012 health facility survey we also paid attention to the role of the private sector.

\section{2: Profile of womenseeking post-abortion care}

Participants in the 2002 and 2012 Health Facilities Survey(HFS) were asked about the demographic characteristics of women attending their health facilities to obtain post-abortion care (PAC) for the treatment of health complications. The respondents were not asked to differentiate between complications from induced and spontaneous abortions. Some changes can be observed between the two studies. In particular, there has been an increase in the proportion of younger women seeking PAC,which possibly reflects improvements intheir care-seeking behaviour. However, while women seeking post-abortion care in 2012 are considered likely to be younger than in 2002 (43\% vs. 31\% aged 15-29), the majority of women in both years fall into the age-group 30 and older (Table 4.1). The respondents in the 2012 survey reported that $92 \%$ of womencoming to their facilities for post-abortion care (PAC)were married (down from 97\% in 2002), $80 \%$ were uneducated or had no formal education (up from $68 \%$ in 2002 ), and $59 \%$ had five or more children (down from 75\%in 2002).

The proportion of HFS respondents reporting that PAC seekers are nulliparous rose from $2 \%$ in 2002 to almost $9 \%$ in 2012 . More than half $(57 \%)$ of the health facility respondentsinterviewed in 2012 were of the opinion that women seeking PAC are usually accompanied by relatives other than their husband and mother-in-law. One-third each reported that husbands or mothers-in-law usuallyaccompaniedthe woman seeking post-abortion treatment, $16 \%$ that the woman would be 
accompanied by her sister, and a similar proportion thatshe would be accompanied by a female friend.

Table 4.1: Characteristics of women most commonly perceived as attending health facilities for treatment of post-abortion complications, Health Facilities Survey, 2002 and 2012

\begin{tabular}{|c|c|c|}
\hline \multirow{2}{*}{ Characteristics: } & 2002 & 2012 \\
\hline & $\%$ & $\%$ \\
\hline \multicolumn{3}{|l|}{ Age group } \\
\hline $15-19$ & 1 & 3 \\
\hline $20-24$ & 10 & 12 \\
\hline $25-29$ & 20 & 28 \\
\hline $30-34$ & 43 & 39 \\
\hline $35-39$ & 20 & 14 \\
\hline 40 or more & 6 & 4 \\
\hline \multicolumn{3}{|l|}{ Marital status } \\
\hline Married & 97 & 92 \\
\hline Single & 3 & 8 \\
\hline \multicolumn{3}{|l|}{ Education } \\
\hline No education & 62 & 74 \\
\hline No formal education & 6 & 6 \\
\hline Primary or less & 11 & 8 \\
\hline Middle or higher & 21 & 12 \\
\hline \multicolumn{3}{|l|}{ No. of children } \\
\hline Nulliparous & 2 & 9 \\
\hline 1 to 2 & 3 & 2 \\
\hline 3 to 4 & 19 & 30 \\
\hline 5 or more & 75 & 59 \\
\hline \multicolumn{3}{|l|}{ Residence* } \\
\hline Urban & - & 29 \\
\hline Rural & - & 71 \\
\hline \multicolumn{3}{|l|}{ Economic status* } \\
\hline Poor & - & 83 \\
\hline Non-poor & - & 17 \\
\hline Total & 100 & 100 \\
\hline \multicolumn{3}{|l|}{ **Usually accompanied* } \\
\hline Husband & - & 33 \\
\hline Mother-in-law & - & 32 \\
\hline Sister & - & 16 \\
\hline Relatives & - & 57 \\
\hline Friends & - & 15 \\
\hline Others & - & 20 \\
\hline No. of facilities & (259) & (266) \\
\hline
\end{tabular}

Source: Health Facilities Survey, unweighted results.

*This information was not collected in the 2002 HFS.

**Multiple responses. 


\section{3: The likelihoodthat women with complications can obtain medical care}

If a woman develops complications from an unsafe abortion, many factors may hinder her from accessing the treatment she needs at a health facility. The possible barriers include cost, distance to travel, the availability of services and family commitments.

The HPS respondentsin 2002 and 2012 were asked the proportion of women experiencing postabortion complications that was likely to be able to obtain medical care.On average, they agreed that non-poor women in urban areaswerethe most likely to get to a health facility for treatment ( $81 \%$ in 2002 and $86 \%$ in 2012), and the rural poor, the least likely (40\% in 2002 and 57\% in 2012). Among the urban poor, these proportions were estimated to be60\% in 2002 and 71\% in 2012, and among the rural non-poor they were estimated at 70\% in 2002 and 77\% in 2012 (Figure 4.1).

Figure 4.1: Proportion of women experiencing abortion-related complications estimated by health professionals to obtain post-abortion care (PAC), by women's residence and economic status, Health Professionals Survey, 2002 and 2012

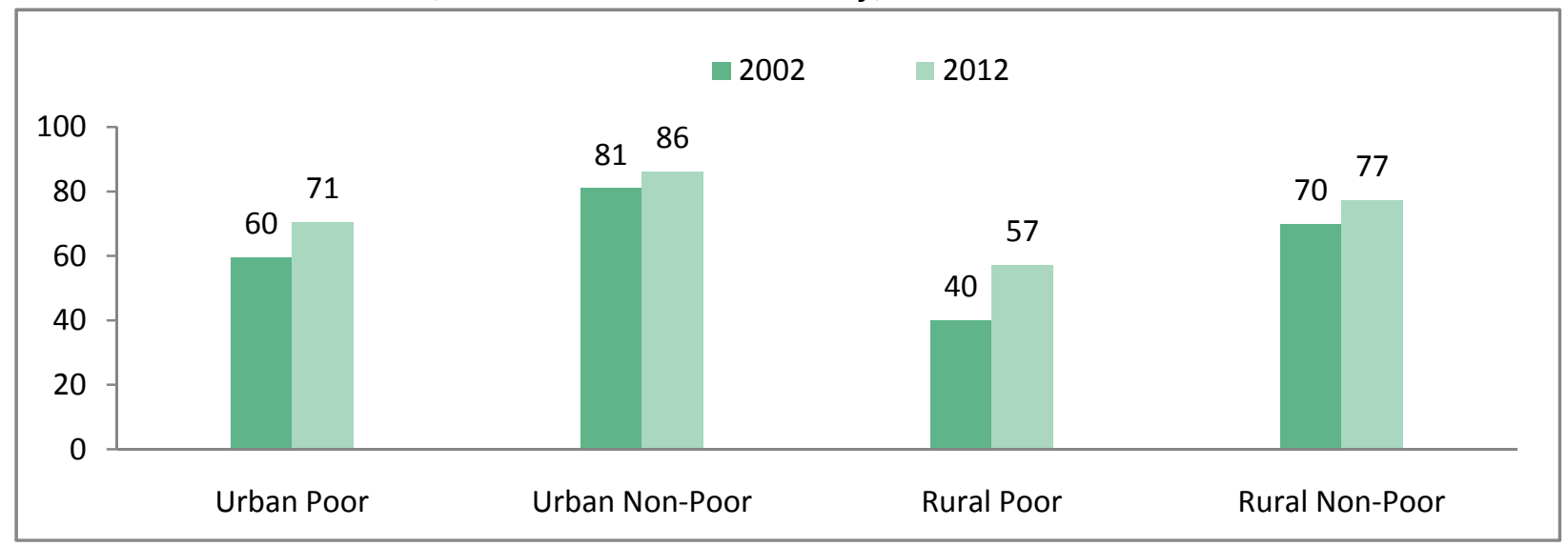

Source: Health Professionals Survey.

Thus, access to PAC services appears to have improved slightly for all four sub-groups of women, but especially for poor rural women. Nevertheless, compared to the other three subgroups, this latter group still lags behind in the proportion who sought care.It is therefore likely that the careseeking behaviour of women in Pakistan has improved overall. However, in 2012, there were some differences in these estimates depending on whether the respondent was a gynaecologist or a health manager or researcher. For example, in the case of poor urban women with abortionrelated complications, $51 \%$ of this last group, compared to $75 \%$ of gynaecologists participating in the HPS, believed that the women would obtain PAC. In the case of the rural poor, these proportions were $40 \%$ and $63 \%$, respectively (Table 4.2 ). 
Table 4.2: Proportion of women experiencing abortion-related complications estimated by health professionals to seek medical treatment, by women's residence and economic status, Health Professionals Survey, 2012

\begin{tabular}{lrrrr|r} 
Current primary profession & Urban Poor & $\begin{array}{r}\text { Urban } \\
\text { Non-Poor }\end{array}$ & Rural Poor & $\begin{array}{c}\text { Rural } \\
\text { Non-Poor }\end{array}$ & \multicolumn{2}{|c|}{ Total } \\
\cline { 2 - 6 } of respondent: & $\%$ & $\%$ & $\%$ & $\%$ & N \\
$\quad$ Qualified gynaecologist & 75 & 88 & 63 & 80 & $(52)$ \\
Doctor & 65 & 79 & 47 & 70 & $(24)$ \\
LHV/Nurse/Midwife/FHT & 75 & 91 & 62 & 83 & $(18)$ \\
$\begin{array}{l}\text { Health } \\
\text { manager/researcher }\end{array}$ & 51 & 86 & 40 & 69 & $(8)$ \\
No. of respondents & $\mathbf{( 7 1 )}$ & $\mathbf{( 8 6 )}$ & $\mathbf{( 5 7 )}$ & $\mathbf{( 7 7 )}$ & $\mathbf{( 1 0 2 )}$
\end{tabular}

Source: Health Professionals Survey.

\section{4: Availability and capacity of PAC services in the public and private sectors}

In both 2002 and 2012 studies, respondents to the Health Facilities Survey (HFS) were asked whether their facility provided post-abortion care (PAC) and about the procedures, medical protocols and services implemented to manage and address women's post-abortion complications. Overall, almost all the respondents reported that their facilities provide PAC services. All the public teaching hospitals, District Headquarter Hospitals (DHQs) and Tehsil Headquarter Hospitals (THQs) offered PAC services, compared to $94 \%$ of the Rural Health Centres (RHCs). One important caveat is that while public-sector facilities were selected through systematic random sampling, the 2012 Health Facility Survey purposively chose a majority of private facilities likely to have a female service provider, on the assumption that these would yield more information about post-abortion care. This may, however, mean that the sample of private facilities suggests a better coverage of services and staffing than is actually available in the country. Public health facilities are mandated to provide PAC services, but this is not the case with private health facilities.

\section{5: Procedures and services used in the treatment of abortion-related complications}

In terms of the PAC methods used, the most dramatic change has been that reported in the use of misoprostol in the treatment of post-abortion complications or incomplete abortion. Reliance on this technique rose from an almost negligible $2 \%$ of facilities in 2002 to $90 \%$ in the 2012 survey (Figure 4.2). 
Figure 4.2: Proportion of respondents at public health facilities reporting the various procedures that may be used to treat post-abortion complications, Health Facilities Survey, 2002 and 2012

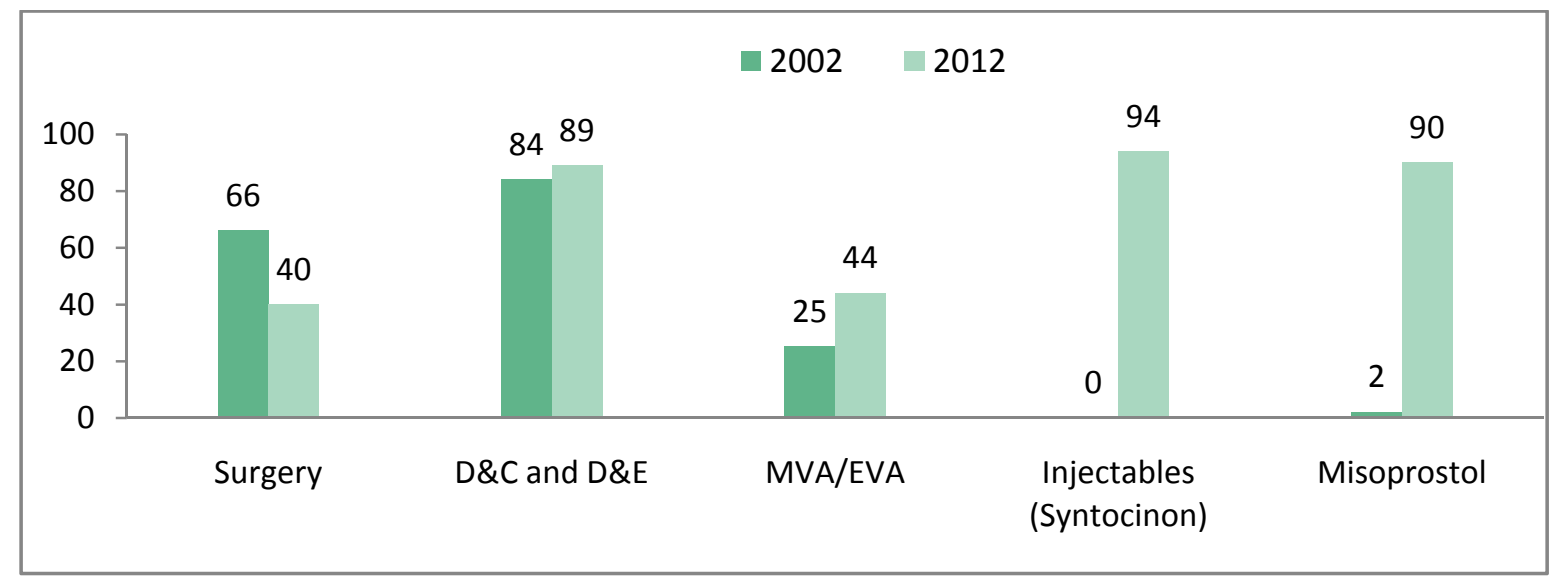

Source: Health Facilities Survey, weighted results.

A similarly high proportionof respondents of public health facilities in both the 2002 and the 2012 HFS (84-89\%) reported the use of D\&C and D\&E* to treat post-abortion complications, despite its invasiveness and relatively high level of medical risk. However, a reduction in the use of surgery (from $66 \%$ in 2002 to $40 \%$ in 2012) to treat abortion related complications is seen. This could possibly be due to a drop in the incidence of more severe complications (such as perforation of the uterus and gut). There has also been an increase in the use of MVA/EVA, from 25\% of facilities in 2002 to $44 \%$ in 2012. Almost all health facilities in both studies reported the use of antibiotics to treat post-abortion complications.

\section{Patterns of treatment in public and private facilities}

In 2012, the most commonly used procedures to treat women for PAC were D\&C/D\&E (58\%) and misoprostol (medication abortion-29\%). Generally, the findings show a fairly similar pattern of treatment in public and private facilities (Figure 4.3). Thirty-nine percent of women attending public facilities were treated by medication abortion (oral, vaginal \& injectable), compared to $23 \%$ at private health facilities. About one half $(52 \%)$ of the women at public facilities were treated by $D \& C / D \& E$,as compared to two-third in the private sector, although the proportion treated through this procedure was comparatively higher for private than for public facilities. (It is of interest that the use of general anaesthesia in the course of a D\&C or D\&E declined between 2002 and 2012, particularly in the public sector.) Very few women in either health sector were treated

\footnotetext{
*Dilatation and curettage $(D \& C)$ is a surgical procedure in which the cervix is dilated and part of the lining of the uterus or contents of the uterus is removed by scrapping. Dilatation and evacuation (D\&E) is a surgical procedure in which the cervix is dilated and the contents of the uterus are evacuated. D\&E is normally used in second trimester abortion. Some providers use the terms D\&C and D\&E interchangeably, and therefore we combine these two methods when discussing results.
} 
by MVA/EVA (public: $3 \%$, private: $7 \%$ ). Only $1 \%$ of PAC cases in public facilities and $3 \%$ in private facilities were treated by abdominal surgery.

Figure 4.3: Percent distribution of women treated for post-abortion care (PAC), by type of procedure, Health Facilities Survey, 2012
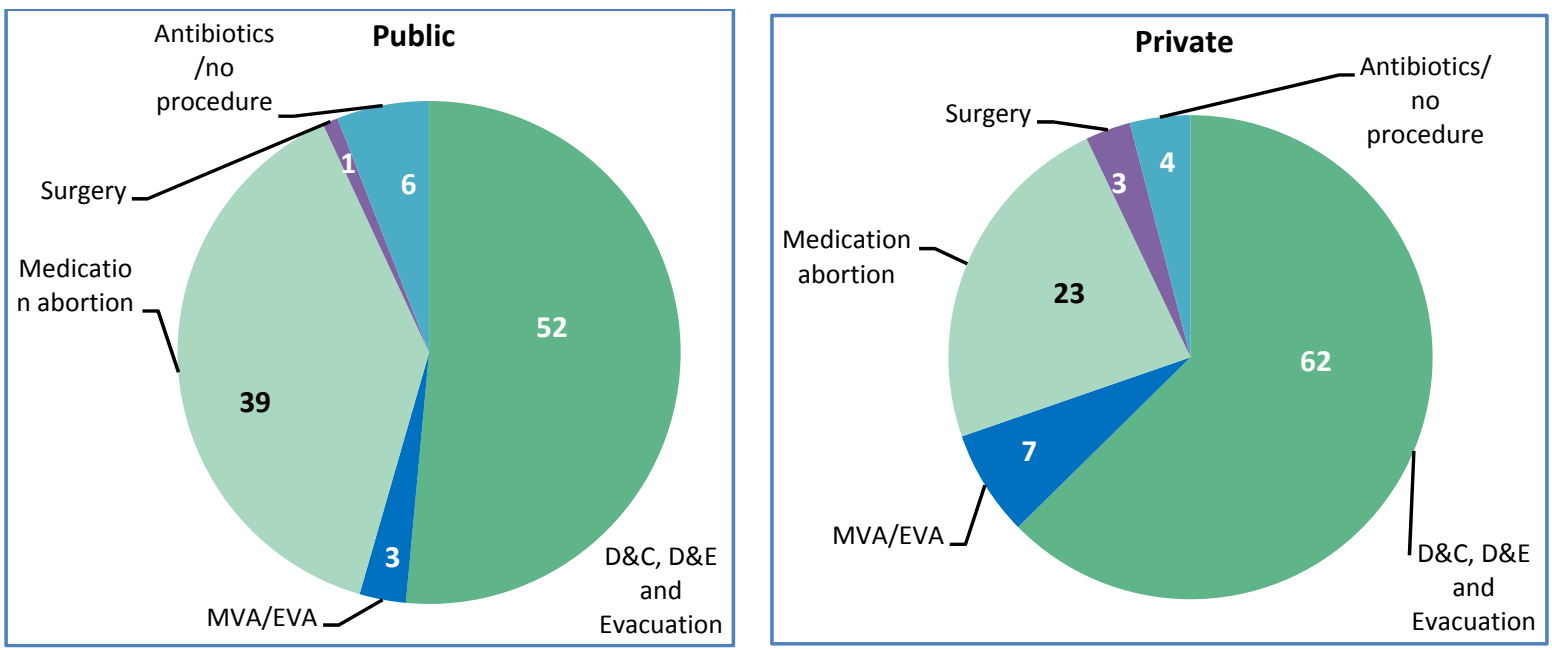

Source: Health Facilities Survey, weighted results.

\section{Treatmentsmost recommended for complications of first-trimester abortion}

The HFS respondents were asked about the treatments that should preferably be used for the complications of a first-trimester abortion, as recommended by official medical safety protocols. Slightly more than half of the respondents said oral or vaginal misoprostolwould be the first choice, $17 \%$ mentioned injectable(Syntocinon)application of this drug, and $27 \%$ ranked D\&C, D\&E and Evacuation first (Figure 4.4).

Figure 4.4: Proportion of respondents saying which treatments would be best recommended for treating a first-trimester PAC case, Health Facilities Survey, 2012.

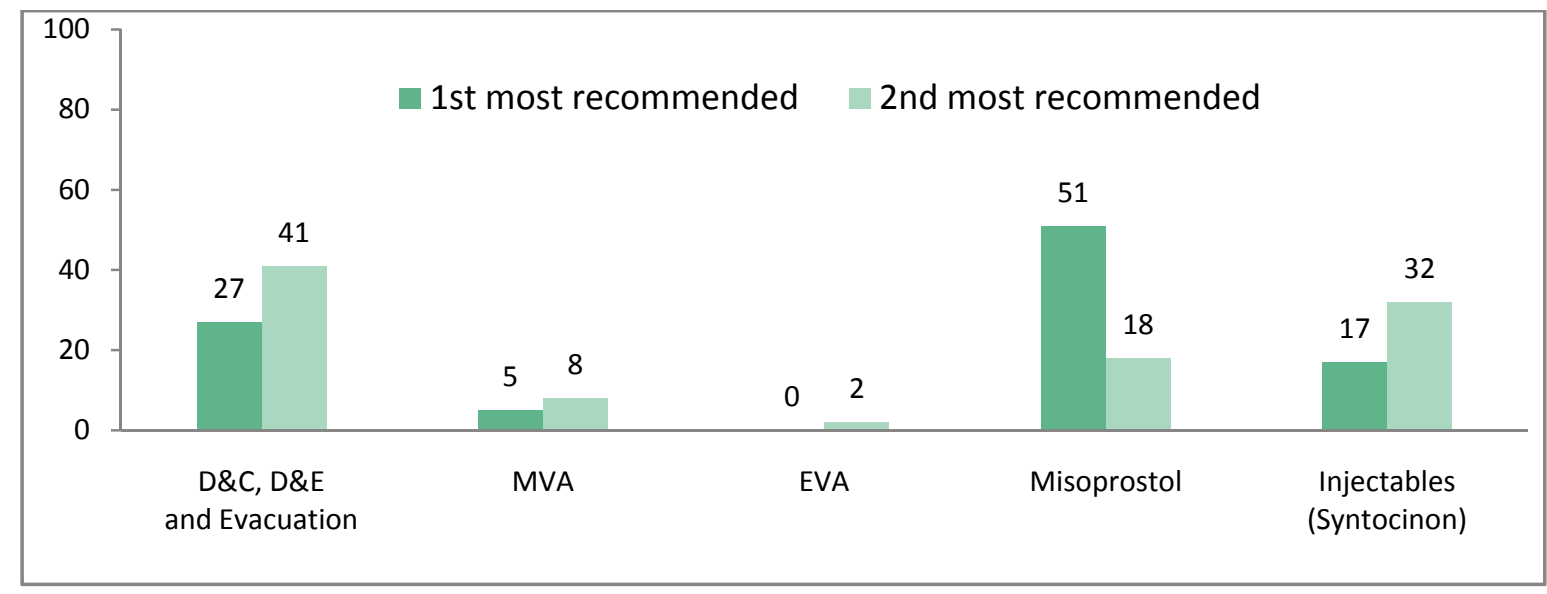

Source: Health Facilities Survey, weighted results. 
Very few respondents said MVA or EVA would be the first choice. Half of the respondents also ranked oral, vaginal or injectable misoprostol as the second choice. The respondents' recommendations were in sharp contrast to currentpost-abortion practices at their health facilities, as shown in Figure 4.3. Vacuum aspiration methods (MVA, EVA) are probably rarely recommended because the service providers do not have this equipment, or have not been trained in the use of these relatively safe and efficient procedures to treat post-abortion complications.

\section{Availability of trained staff}

An inadequate level of human resources is one of the factors underlying the poor performance of health systems intended to deliver effective, evidence-based interventions for priority health problems, and this problem is most critical in developing countries ${ }^{35}$. Although the current study purposively selected private facilities that do provide PAC services, and although all public facilities are supposed to provide these services, many of the health facilities lack sufficient or appropriate numbers of staff trained to treat post-abortion complications. Only $20 \%$ of the public facilities had a gynaecologist on staff, compared to over one-half at the private facilities (Figure 4.5).

Figure 4.5: Proportion of health facilities employing specific types of staff, Health Facilities Survey, 2012.

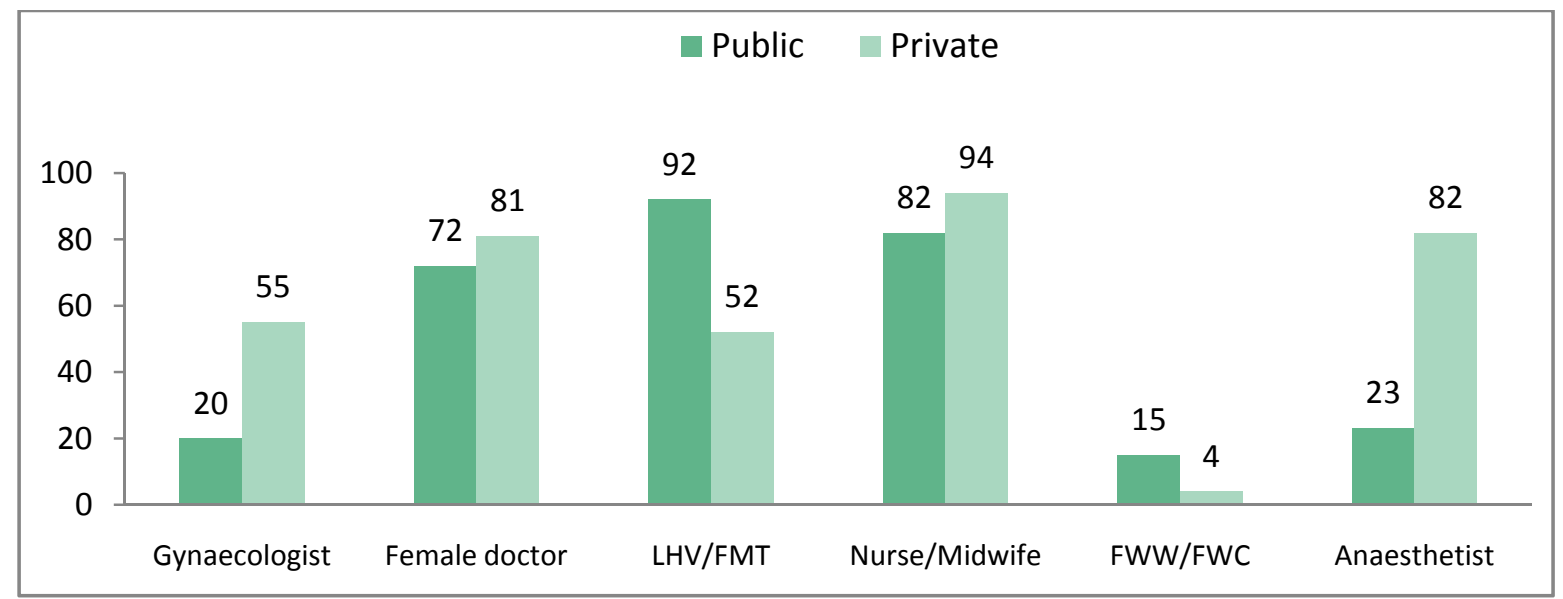

Source: Health Facilities Survey, weighted results.

Government health policy in Pakistanis usually to appoint gynaecologists to work in the highestlevel facilities (public teaching hospitals, followed by DHQ and then THQ hospitals). Female doctors were present in $72 \%$ of the public facilities and $81 \%$ of the private facilities. Less than onequarter of public facilities had an anaesthetist on staff, compared to $82 \%$ of private facilities. Nurses or midwives were almost universally available at public (82\%) and private (94\%) facilities. 
And although a LHV/FMT was available at almost all public health facilities (92\%), only half of the private facilities (52\%) had this type of health professional on staff. Overall, female doctors were available in $77 \%$ of the facilities (public and private combined), while only $41 \%$ had at least one female gynaecologist on staff (Table 4.3).

Table 4.3: Proportion of health facilities with at least one female medical personnel on staff, by type of facility, Health Facilities Survey, 2012

\begin{tabular}{|c|c|c|c|c|c|c|c|c|c|}
\hline & \multicolumn{4}{|c|}{ Public } & \multicolumn{4}{|c|}{ Private } & \multirow[b]{2}{*}{ Total } \\
\hline & Teaching & DHQ & THQ & RHC & Teaching & Large & Medium & Small & \\
\hline & $\%$ & $\%$ & $\%$ & $\%$ & $\%$ & $\%$ & $\%$ & $\%$ & $\%$ \\
\hline Female gynaecologist & 100 & 82 & 30 & 3 & 100 & 59 & 48 & 55 & 41 \\
\hline Female doctor/specialist & 100 & 100 & 88 & 70 & 100 & 100 & 85 & 72 & 77 \\
\hline Female paramedic & 100 & 100 & 100 & 99 & 100 & 100 & 100 & 96 & 98 \\
\hline
\end{tabular}

Source: Health Facilities Survey, weighted results.

All the teaching hospitals in both the public and private sector had a female gynaecologist on staff, as compared to $82 \%$ of DHQs, $30 \%$ of THQs and $3 \%$ of RHCs. Female doctors were present in all the DHQs and private teaching hospitals. However, there were no female doctors in $30 \%$ of the RHCs, and $12 \%$ of the THQs. In contrast, in the private sector, a majority of large hospitals (100\%), medium-sized hospitals (85\%) and small hospitals (72\%) had at least one female doctor. At least one female paramedic was present at almost all the health facilities, both public and private.However, it should be pointed out that the public sector is mandated to provide PAC services and is meant to have at least one female paramedic on its staff. The reason for the almost universal presence of female paramedicsin the private facilities is because the selection of the private-sector sample was tilted towards those facilities that were equipped to provide PAC care.Having a female paramedic on staff was almost a necessary precondition for the selection of facilities in the private sector.

Almost all (97\%) RHCs and 73\% of THQs did not have 24-hour coverage by a gynaecologist, while all thepublic and private teaching hospitals were staffed by a gynaecologist around the clock (Table 4.4). Female doctors were not available around the clockin $37 \%$ of RHCs and in overonequarter of the small hospitals.Almost all public and private facilities either had a nurse/midwife or LHV/FHT on staff. Anaesthetist availability for a full 24 hours was much better overall in the private sector.In $95 \%$ of RHCs, one-quarter of small hospitals, and slightly less than two-thirds of THQs, an anaesthetist was not available throughout the day and night. Finally, 16\% of public RHCs and $12 \%$ of small private hospitals did not have a 24 -hour laboratory technician on staff. The larger the public-sector facility, the more likely it was to have a full-time gynaecologist on staff. 
Table 4.4: Proportion of facilities that do not have 24-hour coverage of staff, according to type of staff and type of facility, Health Facilities Survey, 2012

\begin{tabular}{lrrrr|rrrr|r} 
& \multicolumn{5}{|c|}{ Public } & \multicolumn{4}{|c|}{ Private } \\
\cline { 2 - 7 } & Teaching & DHQ & THQ & RHC & Teaching & Large & Medium & Small & Total \\
Gynaecologist & 0 & 18 & 73 & 97 & 0 & 40 & 52 & 46 & 59 \\
Female doctor & 0 & 0 & 12 & 37 & 0 & 0 & 12 & 28 & 25 \\
Nurse/midwife & 0 & 12 & 5 & 22 & 0 & 0 & 0 & 8 & 10 \\
LHV/FHT & 36 & 34 & 9 & 5 & 37 & 0 & 34 & 53 & 33 \\
Anaesthetist & 0 & 13 & 64 & 95 & 0 & 0 & 0 & 26 & 41 \\
Laboratory technician & 0 & 0 & 4 & 16 & 5 & 0 & 0 & 12 & 10 \\
\hline
\end{tabular}

Source: Health Facilities Survey, weighted results.

There is no little difference in the availability of female doctors and LHV/nurse/midwife across both sectors (Figure 4.6). However, 24-hour availability of a gynaecologist is better at mediumsized and small private hospitals than in the equivalent public-sector facilities (Table 4.4). While the appointment of a gynaecologist is not expected in RHCs, the fact that none are available at three-quarters of THQ hospitals and almost one-fifth of DHQ hospitals is a major barrier for many women needing access to reproductive health services that include post-abortion care.

Figure 4.6: Proportion of public and private facilities that do not have 24-hour coverage, by type of medical staff, Health Facilities Survey, 2012

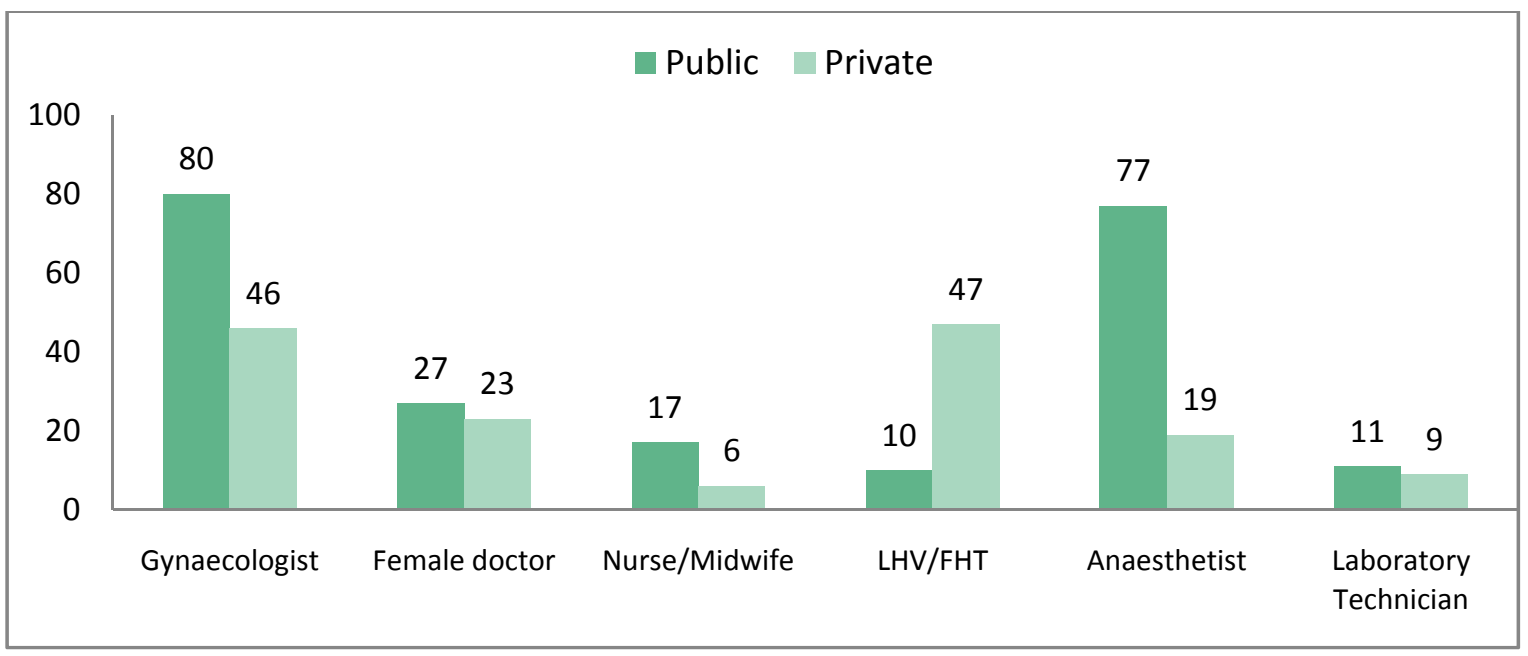

Source: Health Facilities Survey, weighted results. 


\section{Training providers of post-abortion care}

The global shortage of skilled, motivated, and supportive health workers is universally acknowledged as a key development challenge because it is a critical barrier to strengthening health systems, achieving the Millennium Development Goals (MDGs), improving the prospects for universal health coverage, and addressing inequity and poverty ${ }^{36}$. After the findings from the 2002 National Study on unintended Pregnancy and Post-abortion Care were published, the magnitude and seriousness of the personnel and training problem became apparent.Many organisations since then have been conducting training in the provision of safer methods for the management of post-abortion complications. A consultative process was begun by the Population Council, India, to develop a consensus on issues related to the mid-level provision of postabortion care services in four countries of south Asia. In this context, the Population Council's Pakistan office convened a meeting in 2011 at which eminent professionals made the recommendation to train mid-level providers in PAC services. This concept was reiterated by the Council at the $6^{\text {th }}$ Asia Pacific Conference Reproductive and Sexual Reproductive Health and Rights in Indonesia in 2011.

Within this new policy context, health facility staff were asked about the number and type of providers in their institutions who had been trained in different methods of PAC management within last two years.Table 4.5 shows that overall, 1,333 providers were trained in the 266 facilities visited by the teams in all the four provinces covered by the study. The vast majority (90\%) of this training took place in Punjab (670 providers) and Sindh (542); only $10 \%$ of the providers trained were from KPK (47) and Balochistan (74). The maximum numbers of providers were trained in family planning and MVA.

Table 4.5: Number of doctors and nurses who received training in various PAC-related procedures, by province, Health Facilities Survey, 2012

\begin{tabular}{|c|c|c|c|c|c|c|}
\hline Procedures & Service providers & Punjab & Sindh & KPK & Balochistan & Total \\
\hline \multirow{2}{*}{ MVA } & Doctor & 174 & 213 & 27 & 26 & 440 \\
\hline & Nurse & 4 & 20 & 0 & 2 & 26 \\
\hline \multirow{2}{*}{ EVA } & Doctor & 42 & 34 & 3 & 0 & 79 \\
\hline & Nurse & 0 & 0 & 0 & 0 & 0 \\
\hline \multirow{2}{*}{ Misoprostol } & Doctor & 88 & 26 & 1 & 0 & 115 \\
\hline & Nurse & 8 & 4 & 0 & 0 & 12 \\
\hline \multirow{2}{*}{ FP/Birth spacing } & Doctor & 282 & 167 & 16 & 33 & 498 \\
\hline & Nurse & 72 & 78 & 0 & 13 & 163 \\
\hline Total & & 670 & 542 & 47 & 74 & 1,333 \\
\hline
\end{tabular}

Source: Health Facilities Survey, unweighted results. 
Table 4.6 shows by the number and type of providers trained in various procedures in both public and private health facilities. The major concentration of training occurredin the public sector and in higher-level facilities. It is interesting thattwo-thirds (66\%) of training in all procedures was conducted in teaching hospitals, of which only $16 \%$ are in the private sector. Very few providers workingin RHCs received training, even in family planning,despite the fact that a substantial number of PAC cases go to these institutions for treatment. The RHCs are mostly staffed by midlevel providers, whose skills in post-abortion care need much improvement, since this area is not part of their regular training curriculum.

Table 4.6: Number of doctors and nurses who received training in various PAC-related procedures, by type of facility, Health Facilities Survey, 2012

\begin{tabular}{|c|c|c|c|c|c|c|c|c|}
\hline \multirow[b]{2}{*}{ Procedures } & \multirow{2}{*}{$\begin{array}{l}\text { Service } \\
\text { providers }\end{array}$} & \multicolumn{4}{|c|}{ Public } & \multicolumn{2}{|c|}{ Private } & \multirow[b]{2}{*}{ Total } \\
\hline & & Teaching & DHQ & THQ & RHC & Teaching & Others & \\
\hline \multirow{2}{*}{ MVA } & Doctor & 300 & 68 & 7 & 4 & 34 & 27 & 440 \\
\hline & Nurse & 11 & 10 & 4 & 0 & 0 & 1 & 26 \\
\hline \multirow{2}{*}{ EVA } & Doctor & 37 & 15 & 0 & 1 & 23 & 3 & 79 \\
\hline & Nurse & 0 & 0 & 0 & 0 & 0 & 0 & 0 \\
\hline \multirow{2}{*}{ Misoprostol } & Doctor & 67 & 29 & 3 & 2 & 7 & 7 & 115 \\
\hline & Nurse & 4 & 0 & 4 & 1 & 0 & 3 & 12 \\
\hline \multirow{2}{*}{ FP/Birth spacing } & Doctor & 307 & 64 & 42 & 17 & 28 & 40 & 498 \\
\hline & Nurse & 11 & 28 & 44 & 17 & 48 & 15 & 163 \\
\hline Total & & 737 & 214 & 104 & 42 & 140 & 96 & 1,333 \\
\hline
\end{tabular}

Source: Health Facilities Survey, unweighted results.

The HFS respondents were asked about the duration of training given for various types of PACrelated procedures. Training for doctors in any single procedure varied widely in duration (Figure 4.7). Around $80 \%$ of doctor training in MVA andmisoprostol, $57 \%$ of training in family planning and $44 \%$ of training in EVA lasted 1-5 days. Training of 6-10 days duration was provided to $13 \%$ of doctors in MVA, $30 \%$ in EVA, $18 \%$ in misoprostol and $23 \%$ in family planning. These data show wide variations in the length of training of doctors for the same type of procedure. Ideally, there should be a uniform curriculum for each type of procedure and the duration of training should be standardized. 
Figure 4.7: Proportion of doctors who have received training in various PAC-related procedures, by duration of their training, Health Facilities Survey, 2012

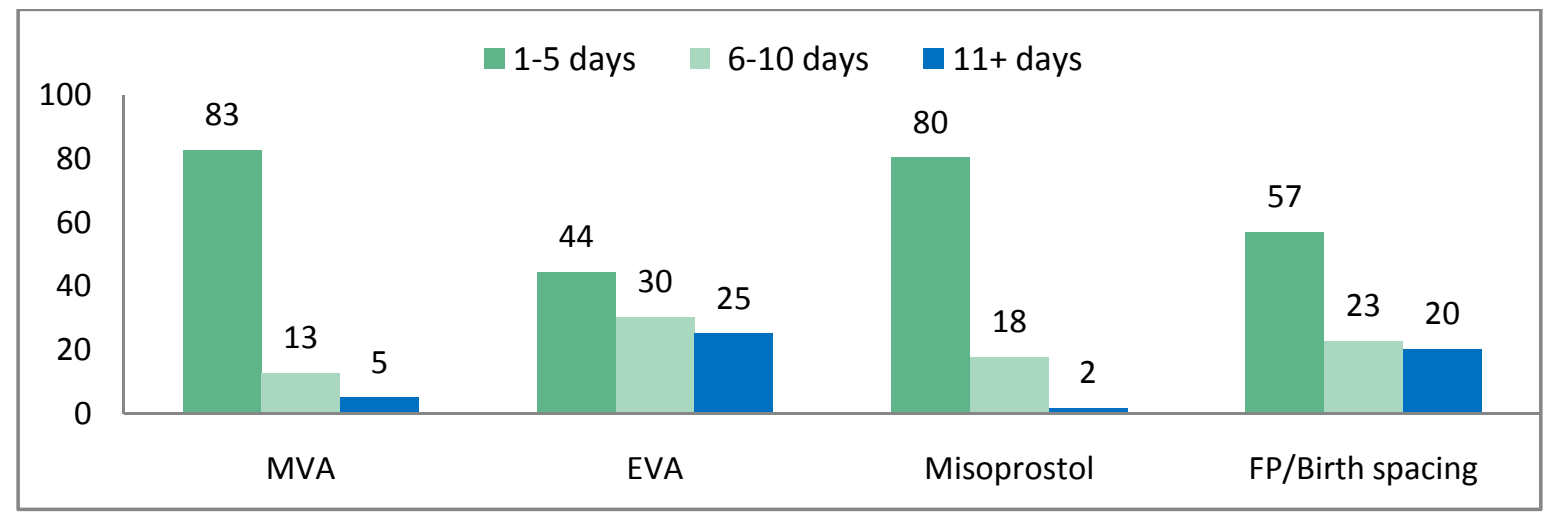

Source: Health Facilities Survey, weighted results.

\section{Availability of PAC equipment}

Themanagement of post-abortion complications, whether as a result ofspontaneous or an induced abortion, involves removing the products of conception. MVA has been reported to be safe and effective in such cases. The efficacy of MVA is comparable to that of EVA, with completion rates in most studies of $98 \%$ or greater. MVA offers an acceptable alternative to either D\&C or EVA ${ }^{37}$. Since the availability of equipment is a necessary requirement for the provision of PAC services, respondents in the surveyed health facilities were asked about the availability of MVA, EVA kits and D\&C sets in theirinstitutions.

All the public and private teaching hospitals had D\&C sets, and more than two-thirds had EVA kits. MVA kits were available in more than half (54\%) of the public, compared to $42 \%$ of the private teaching hospitals (Table 4.7) D\&C sets were also available in almost all public sector and all small private hospitals. However availability of D\&C sets at private medium-sizedand large hospitals was $88 \%$ and $60 \%$, respectively. On the other hand, MVA kits were limited-available in less than one-fifth of DHQs, THQs and RHCs, $34 \%$ ofprivate medium-sizedhospitals and $28 \%$ of small hospitals. 
Table 4.7: Proportion of facilities with functioning PAC equipment, by type of facility, Health Facilities Survey, 2012

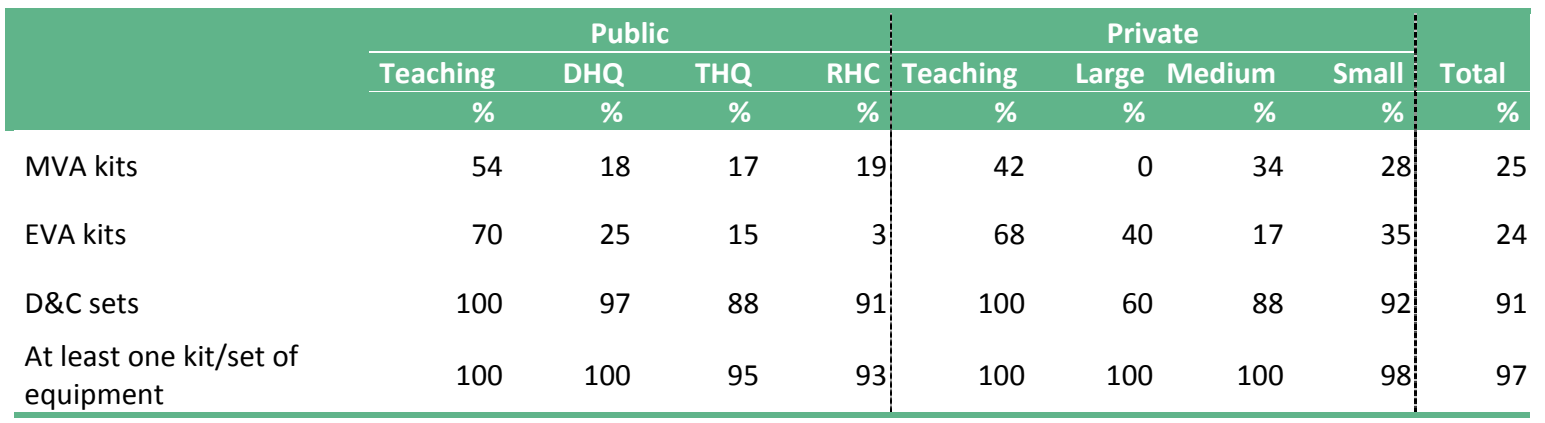

Source: Health Facilities Survey, weighted results.

Respondents were asked about the availability in their facilities of a range of equipment, medicines and supplies needed in the provisionof post-abortion care. Misoprostol was more likely to be available in the private sector facilities and was of more limited availability in the public sector facilities ( $89 \%$ compared to $54 \%$, data not shown). Nearly all public teaching hospitals (91\%), private teaching hospitals (95\%), private large hospitals (100\%), private medium-sized hospitals (88\%) and small hospitals (89\%) reported the availability of misoprostol (Table 4.8). Moreover, $60 \%$ of DHQs and THQs, and half of RHCs, also reported that misoprostol was available in their facilities for post-abortion care purposes.

Table 4.8: Proportion of health facilities equipped with standard post-abortion care (PAC) supplies, by type of facility, Health Facilities Survey, 2012

\begin{tabular}{|c|c|c|c|c|c|c|c|c|c|}
\hline & \multicolumn{4}{|c|}{ Public } & \multicolumn{4}{|c|}{ Private } & \multirow[b]{2}{*}{ Total } \\
\hline & Teaching & DHQ & THQ & RHC & Teaching & Large & Medium & Small & \\
\hline & $\%$ & $\%$ & $\%$ & $\%$ & $\%$ & $\%$ & $\%$ & $\%$ & $\%$ \\
\hline Sterilizer/boiler & 97 & 88 & 78 & 72 & 95 & 100 & 100 & 94 & 88 \\
\hline Autoclave & 100 & 100 & 63 & 58 & 95 & 60 & 100 & 91 & 82 \\
\hline Bleach/chlorine solution & 85 & 99 & 69 & 46 & 91 & 100 & 100 & 94 & 81 \\
\hline Broad spectrum antibiotics & 100 & 100 & 92 & 91 & 95 & 100 & 100 & 98 & 96 \\
\hline Analgesics & 100 & 79 & 81 & 90 & 95 & 100 & 100 & 97 & 94 \\
\hline Misoprostol & 91 & 60 & 60 & 50 & 95 & 100 & 88 & 89 & 76 \\
\hline Uterotonic drugs & 100 & 99 & 72 & 77 & 95 & 100 & 100 & 85 & 85 \\
\hline Plasma expanders & 100 & 100 & 61 & 51 & 95 & 81 & 97 & 89 & 79 \\
\hline Blood transfusion & 100 & 100 & 65 & 32 & 95 & 100 & 97 & 78 & 70 \\
\hline Functional ambulance & 100 & 99 & 96 & 85 & 95 & 100 & 11 & 21 & 49 \\
\hline
\end{tabular}

Source: Health Facilities Survey, weighted results. 
The proper management of post-abortion complications also requires other essential equipmentsterilizing arrangements, blood transfusion and ambulance services, and medicines such as antibiotics pain-relieving drugs, misoprostol, uterotonic drugs*, and plasma expanders ${ }^{* *}$. All the public teaching and DHQ hospitals and all the private medium-sized hospitals, $95 \%$ of the private teaching hospitals, $91 \%$ of the private small hospitals and $60 \%$ of the private large hospitals had autoclaves available,compared to $63 \%$ of THQs and $58 \%$ of RHCs (Table 4.8). Bleach (chlorine solution) is essential for disinfection of instruments. A majority of all public and private facilities had chlorine solution available.However, it was not available at more than half of the RHCs and slightly less than one-third of the THQ hospitals.

Antibiotics, pain-relieving drugs, uterotonic drugs and plasma expanders were reported as being almost universally available in all the health facilities. However, only half of the RHCs and less than two-thirds of the THQs had plasma expanders in stock. Similarly, almost everyteaching hospital and largehealth facility reported the availability of blood transfusion arrangements, compared to one-third of RHCs and two-thirds of THQs. Functioning ambulances were available in almost all the public teaching, DHQ and THQ hospitals, in all private large hospitals, in 95\% of private teaching hospitals and in $85 \%$ of RHCs, as compared to only $11 \%$ ofprivate medium-sized hospitals and $21 \%$ of small hospitals.

\footnotetext{
* Drugs that cause contraction of the uterus

** Intravenous solutions of a substance (for example, dextran) used as a substitute for plasma for transfusion in case of haemorrhage or shock.
} 


\section{Chapter 5 Post-Abortion Care Service Statistics}

This chapter describes the caseload of women treated for post-abortion complications at the health facilities included in the Health Facilities Survey (HFS). The caseloads include both outpatients and in-patientsreceiving treatment for spontaneous and induced abortions. The information is used to estimate the number and rate of post-abortion complications treated at facilities at the national and provincial levels across Pakistan. It should be pointed out once again that while we expect to have captured the full range of facilities in the public sector, in the case of the private sector we only sampled the facilities that were definitely providing PAC services. Therefore, as a caveat it should be emphasized that we are presenting the 'best' range of quality of care for PAC services in the private sector.Moreover, a substantial proportion of private-sector facilities are in fact not serving PAC clients, even though they may have the potential to do so at some later point.

\section{1: Assessing the quality of the data}

In this section we describe thevarious ways used to estimatethe numbers of women who present themselves at the health facilities and receive treatment for post-abortion complications. The sample on which these numbers are based include tertiary, secondary and primary health care facilities in both the public and private sectors. Since most of the facilities either do not regularly record information about post-abortion complications, or this information is incomplete (see the list of facilities recording health statistics on post-abortion care and a flow chart in Annex 5a and Annex 5b), weused three approachesto collect these estimates. The respondents selected to provide these estimates were experienced physicians working at the health facilities. For larger hospitals (teaching hospitals, DHQs and THQs and their equivalent private facilities), respondents were gynaecologists or female doctors from the gynaecology and obstetrics department. For the smaller (RHC) facilities, either female doctors or LHVs/Nurses/FMTs were interviewed; and in a few cases, staff members who were in charge of the facility were asked these questions.

First, respondents at each health facility were asked to provide an estimate of the number of PAC cases treated in the facility during an average month, separately for out-patients and in-patients. These estimates were then multiplied by 12 to produce an estimated caseload in an average year. Secondly, the health facility staff were asked to estimate the number of PAC cases treated in the past month, separately for out-patients and in-patients. Again the numbers were multiplied by 12 
to obtain a second estimate of that facility's annual caseload. Thirdly, we asked whether the facility compiled statistics on post-abortion care: if aggregate statistics were available, the interviewer extracted data items specified in the HFS questionnaire.To minimize fluctuations in the yearly figures, questions were posed about an average year. It appears that across all the provinces, recall of the number for an average year was higher than the estimation for the past year. Overall, 19\% more estimated cases were reported for an average year than for the past year; and 23\%more in-patients and $13 \%$ moreout-patients were estimated in the course of an average year than in the course of a past year. Thedifference between the two estimates was wider for Balochistan and KPK than for the other two provinces (see the hospital statistics data in Annex 6, Table 2A).

A small proportion of facilities (18\%) reported that hospital statistics on post-abortion cases were not collected. The majority of hospitals and health outlets did keep these records, but the available data were sometimes incomplete. The outpatient or inpatient records of one-thirdof the health facilities were missing. Because of the incompleteness of this data source the statistics from facility records were not considered sufficiently comprehensive to be used inestimating the number of PAC cases treated in each facility, while estimates based on the average year and the past year are comparatively complete, consistent and reliable. We therefore decided to take the mean of the two standard estimates-the number treated in the average year and the number treated in the past year-as the best estimate for each facility. The data presented in the following sections are based on the mean of these two estimates.

\section{2: Differentials in annual PAC caseload by type of facility and by health sector}

Table 5.1 presents estimates of the total number of PAC cases treated at public and private health facilities in the four major provinces of Pakistan in 2012. The data were weighted to adjust for all the health facilities in the four provinces(see the application of weights for public and private health facilities in Annex 7; and standard errors (SE) in Annex 10, Table A4). 
Table 5.1: Annual numbers of PAC patients treated as out andin-patients, by health sector and type of facility, Health Facilities Survey, 2012

\begin{tabular}{|c|c|c|c|c|c|c|c|c|c|c|c|c|c|c|c|c|c|c|}
\hline & \multicolumn{8}{|c|}{ Public } & \multicolumn{8}{|c|}{ Private } & \multirow{2}{*}{\multicolumn{2}{|c|}{ Total }} \\
\hline & \multicolumn{2}{|c|}{ Teaching } & \multicolumn{2}{|c|}{ DHQ } & \multicolumn{2}{|c|}{ THQ } & \multicolumn{2}{|c|}{ RHC } & \multicolumn{2}{|c|}{ Teaching } & \multicolumn{2}{|c|}{ Large } & \multicolumn{2}{|c|}{ Medium } & \multicolumn{2}{|c|}{ Small } & & \\
\hline & UW & W & UW & W & UW & W & UW & W & UW & W & UW & W & UW & W & UW & W & UW & W \\
\hline Mean no. of out-patients per facility & 1085 & 1,059 & 348 & 293 & 257 & 250 & 104 & 102 & 309 & 308 & 593 & 674 & 145 & 155 & 179 & 175 & 317 & 191 \\
\hline Total no. of out-patients & 35,796 & 37,248 & 8,358 & 31,490 & 11,318 & 38,685 & 6,558 & 63,878 & 6,492 & 9,933 & 2,370 & 39,712 & 1,734 & 47,052 & 11,616 & 188,974 & 84,242 & 456,973 \\
\hline Mean no. of in-patients per facility & 682 & 681 & 307 & 305 & 85 & 82 & 41 & 42 & 225 & 224 & 201 & 243 & 117 & 83 & 92 & 90 & 185 & 100 \\
\hline Total no of in-patients & 22,513 & 23,973 & 7,356 & 32,770 & 3,729 & 12,646 & 2,604 & 25,985 & 4,734 & 7,212 & 804 & 14,287 & 1,398 & 25,155 & 6,008 & 96,861 & 49,146 & 238,889 \\
\hline $\begin{array}{l}\text { Mean no. of out and in-patients } \\
\text { per facility }\end{array}$ & 1767 & 1,740 & 655 & 599 & 342 & 331 & 145 & 144 & 535 & 532 & 794 & 917 & 261 & 238 & 271 & 265 & 501 & 291 \\
\hline Total no. of out and in-patients & 58,309 & 61,222 & 15,714 & 64,259 & 15,047 & 51,331 & 9,162 & 89,863 & 11,226 & 17,145 & 3,174 & 53,999 & 3,132 & 72,207 & 17,624 & 285,835 & 133,388 & 695,861 \\
\hline
\end{tabular}

Source: Health Facilities Survey, unweighted (UW) and weighted (W) results.

Note: Mean of average per year and past-year estimates. 
An estimate ofapproximately 696,000 PAC cases were treated in the four provinces in 2012457,000 as out-patients and240,000 as in-patientsOf the total of 696,000 (SE $=63,357,95 \%$ $\mathrm{Cl}=570,148-821,574) \mathrm{PAC}$ cases (including both induced and spontaneous abortions), 429,000 ( $S E=595,59,95 \% \mathrm{Cl}=311,012-547,362$ ) were served by the private sector and 267,000 (SE=21606, 95\% $\mathrm{Cl}=223805-309545$ )by the public sector (Figure 5.1)(see SE and 95\% confidence interval(Cl) inAnnex 10, Table A5).

Figure 5.1: Total annual number of PAC patients treated in health facilities, by type of facility, Health Facilities Survey, 2012

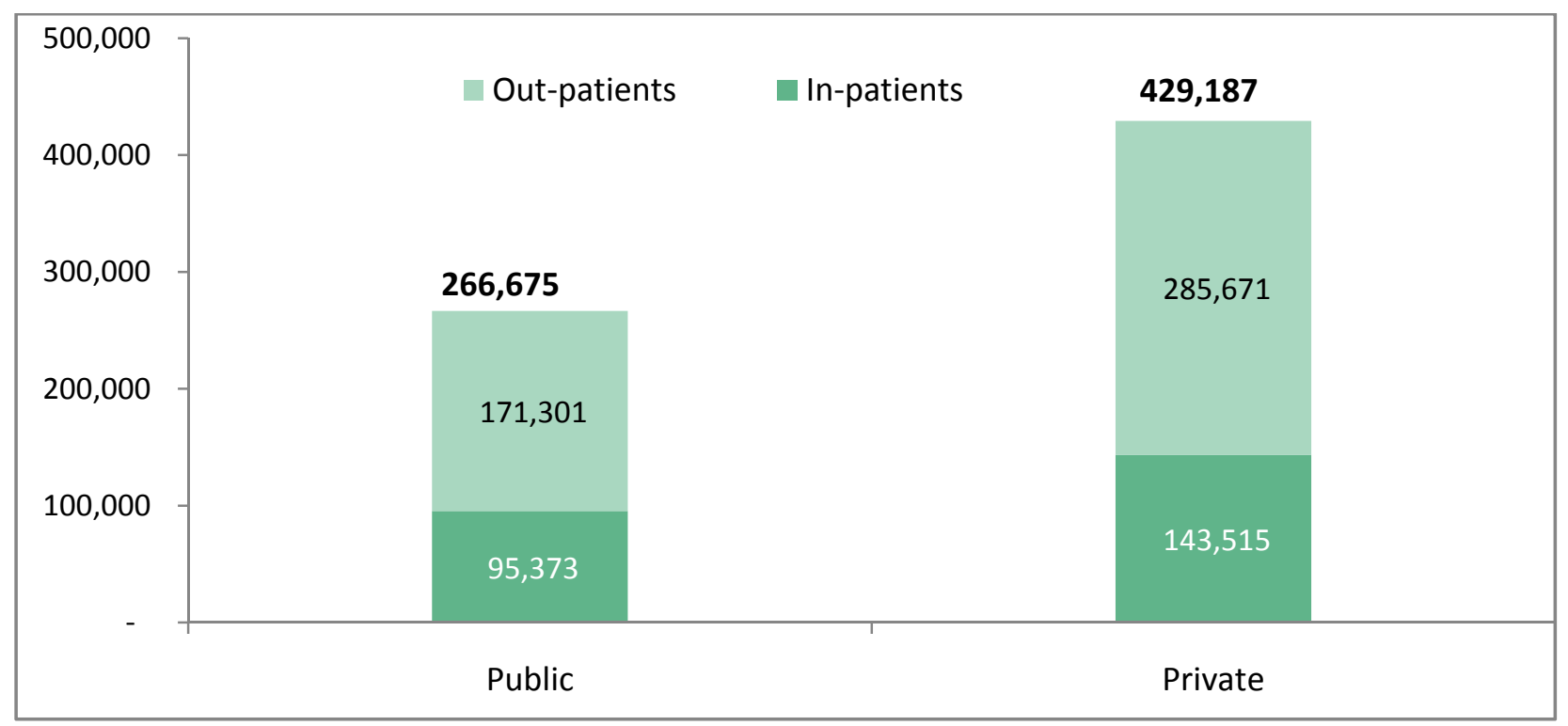

Source: Health Facilities Survey, weighted results.

The highest total number of cases were treated by private small hospitals and by rural health centres (RHCs); 286,000 and 89,900 per year, respectively. This is followed by private mediumsized hospitals,with72,000 cases a year. Public teaching hospitals and District Headquarters Hospitals (DHQs) had an overallcaseload of around 61,000 and 64,000, and private-sector large hospitals treated54,000a year-similar to the number treated inTehsil Hospitals (THQs)-51,300. The lowest caseload was observedin private teaching hospitals-17,000.

On average, each facility treated 291 post-abortion cases, 191 as out-patients and 100 as inpatients, that is, about one inpatient for every two out-patients, a ratio that was similar for public and for private facilities (Table 5.1). As expected, the larger thebeds size in a facility, the higher the average PAC caseload. The mean number of outpatient PAC cases was highest for public teaching hospitals (1,740), followed by private large hospitals(917, 95\% Cl=796-1038), private teaching hospitals $(532)$ and DHQs $(599,95 \% \mathrm{Cl}=382-815)$. The smallest mean numbers of cases wereestimated for RHCs $(144,95 \% \mathrm{Cl}=106-181)$, private small hospitals (265, 95\% Cl=190-340) 
and private medium-sized hospitals (238, 95\% Cl=95-381)(see SE and $95 \% \mathrm{Cl}$ in Annex 10, Table A4).

Overall, the private sector accounts for $62 \%$ of all PAC cases treated, while the public sector treats the remaining $38 \%$. Within the private sector, small facilities treat are largely responsible for most PAC care, accounting for around two-thirds of patients treated. They are followed by medium-sized facilities (17\%), large facilities (13\%) and private teaching hospitals (4\%). In the public sector, RHCs treat around 34\% of PAC patients, followed by DHQs (24\%), public teaching hospitals (23\%) and THQs (19\%)

\section{3: Differentials inannual PAC caseload by province}

Overall, Sind province has the highest mean number of PAC cases per facility (645), followed by Punjab (470) and KPK (412). Balochistan province has the lowest average annual PAC caseload per facility (304 cases). Public-sector facilities in all provinces have a higher mean caseload than do private-sector facilities. The inclusion of metropolitan Karachi in the HFS for Sindh could be the reason for the much higher average caseloads seen for Sindh province.

Punjab has the highest annual caseload of PAC patients, with around 416,000 (SE=69,453, 95\% Cl= 278,624-554,242) women treated for post-abortion complications in 2012. It is followed by Sindh $(175,000, \mathrm{SE}=35,278,95 \% \mathrm{Cl}=104,909-244,907), \mathrm{KPK}(57,000, \mathrm{SE}=20,531,95 \% \mathrm{Cl}=16,421-97,897)$ and Balochistan (47,000, SE=16958, 95\% Cl=13712-81010) (see $\mathrm{SE}$ and $95 \% \mathrm{Cl}$ in Annex 10, Table A6).

In both Punjab and Sindh, PAC is predominantly treated in the private sector (58\% and $70 \%)$, while in KPK and Balochistan it plays a smaller role (33\% and 39\%)(Figure 5.2). 
Figure 5.2: Annual caseload of PAC patients, by public and private sector and by province, Health Facilities Survey, 2012

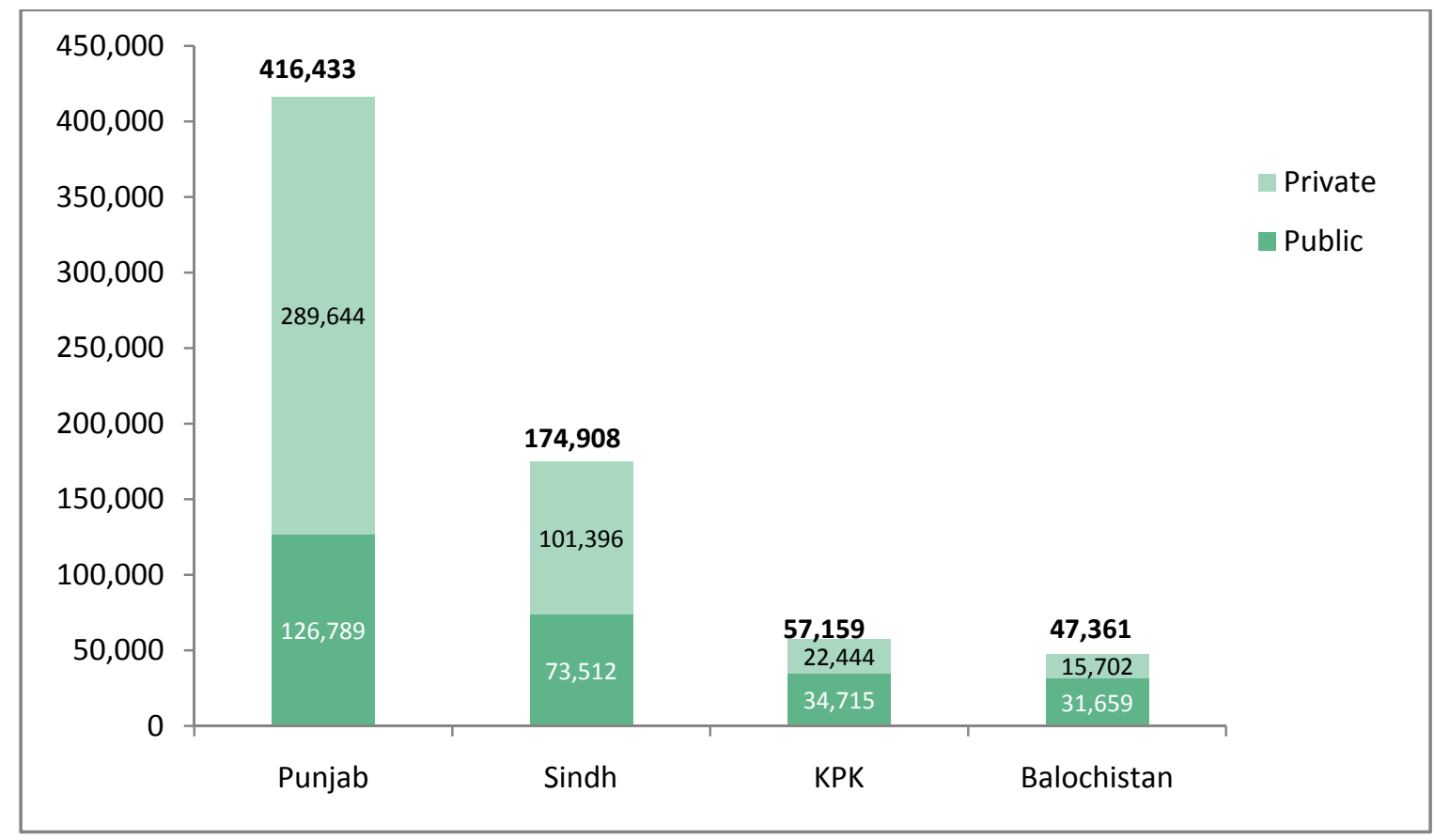

Source: Health Facilities Survey, weighted results.

Note: The weights are shown in Annex 7, Table A3.

In every province, the average annual caseload of PAC cases is largest for public teaching hospitals (Table 5.2). In Punjab and Sindh, private large hospitals have the second largest caseload. In KPK, the second largest annual average numbers are found in DHQs, and in Balochistanthey are found in THQ hospitals. For Sindh and Balochistan, DHQs have the third largest caseload, compared to private teaching hospitals for Punjab and private medium size hospitals for KPK. In Punjab,private large hospitals treat the highest proportion of out-patients(75\%), in Sindh and KPK, RHCs do (78\% and 98\%, respectively), and in Balochistan, THQs do (94\%). The smallest proportion of patients treated as out-patients were found in DHQs in KPK (16\%), in private teaching hospitals in Sindh (49\%), in DHQs and private small hospitals in KPK (17\%) and in RHCs in Balochistan (58\%). There were no private large hospitals in KPK and Balochistan. 
Table 5.2: Annual mean caseload of PAC (out and in-patients) by type of facility, health sector and province, Health Facilities Survey, 2012

\begin{tabular}{|c|c|c|c|c|c|c|c|c|c|c|}
\hline \multirow{3}{*}{$\begin{array}{l}\text { Average annual no. of PAC } \\
\text { cases per facility: }\end{array}$} & \multicolumn{9}{|c|}{ Type of facilities } & \multirow[b]{3}{*}{ Total* } \\
\hline & \multicolumn{4}{|c|}{ Public } & \multicolumn{4}{|c|}{ Private } & \multirow[b]{2}{*}{ Total } & \\
\hline & Teaching & DHQ & THQ & RHC & Teaching & Large & Medium & Small & & \\
\hline \multicolumn{11}{|l|}{ Punjab } \\
\hline Out-patients & 700 & 298 & 333 & 103 & 505 & 705 & 157 & 206 & 293 & 208 \\
\hline In-patients & 596 & 311 & 119 & 51 & 284 & 234 & 76 & 85 & 177 & 96 \\
\hline Out and in-patients & 1,296 & 610 & 452 & 155 & 789 & 939 & 233 & 291 & 470 & 304 \\
\hline$\%$ out-patients & 54 & 49 & 74 & 67 & 64 & 75 & 67 & 71 & 62 & 68 \\
\hline \multicolumn{11}{|l|}{ Sindh } \\
\hline Out-patients & 2,201 & 501 & 188 & 141 & 212 & 570 & 159 & 177 & 443 & 217 \\
\hline In-patients & 856 & 252 & 79 & 40 & 217 & 300 & 81 & 102 & 201 & 105 \\
\hline Out and in-patients & 3,057 & 753 & 267 & 181 & 430 & 870 & 240 & 278 & 645 & 322 \\
\hline$\%$ out-patients & 72 & 67 & 70 & 78 & 49 & 66 & 66 & 64 & 69 & 67 \\
\hline \multicolumn{11}{|l|}{ KPK } \\
\hline Out-patients & 587 & 120 & 71 & 60 & 209 & - & 0 & 40 & 160 & 77 \\
\hline In-patients & 843 & 642 & 10 & 1 & 144 & - & 540 & 195 & 251 & 162 \\
\hline Out and in-patients & 1,429 & 762 & 81 & 61 & 353 & - & 540 & 234 & 412 & 238 \\
\hline$\%$ out-patients & 41 & 16 & 88 & 98 & 59 & - & 0 & 17 & 39 & 32 \\
\hline \multicolumn{11}{|l|}{ Balochistan } \\
\hline Out-patients & 576 & 336 & 470 & 75 & - & 390 & - & 104 & 238 & 152 \\
\hline In-patients & 228 & 100 & 32 & 54 & - & 36 & - & 22 & 65 & 45 \\
\hline Out and in-patients & 804 & 436 & 502 & 129 & - & 426 & - & 126 & 304 & 197 \\
\hline$\%$ out-patients & 72 & 77 & 94 & 58 & - & 92 & - & 83 & 78 & 77 \\
\hline No. of facilities & (33) & (24) & (44) & (63) & (21) & (4) & (12) & (65) & (266) & (266) \\
\hline
\end{tabular}

Source: Health Facilities Survey, Unweighted results.

*Weighted results (Total)

Note: There are no private teaching hospitals and private medium-size hospitals in Balochistan and no private large hospitals in KPK.

Sindh has the largest mean caseload per facility among the public teaching hospitals, followed byKPK, Punjab and Balochistan(Table 5.2). Most of the teaching hospitals in Sindh are located in the metropolitan city of Karachi. These are very large hospitals and cater to the residents of this mega-city, which is the probable reason why this province has the largest mean caseload at public teaching hospitals. The caseload at DHQs is largest in KPK province, followed by Sindh, Punjab and Balochistan. The THQ hospitals in KPK had the lowest average caseload, and those in Balochistan, the highest, followed closely by Punjab. The average annual caseload for RHCs was highest in Sindh, followed by Punjab, Balochistan and KPK. This finding could reflect the improved services being provided in RHCs in Sindh and Punjab provinces, which are now being managed through thePeoples Primary Healthcare Initiative (PPHI) begun by the Punjab Rural Support Program 
(PRSP). In an effort to improve delivery of basic health services, the government of Pakistan has contracted a group of government managers to managelower-level health facilities. This is a unique model that gives managers more authority to make decisions. Increased personal accountability, a need for managers to prove themselves, and their ability to make management decisions, including staff hiring and transfers, has enabled the new managers to improve the availability of medicines and supplies, hire doctors on contract, and improve provider performance through increased monitoring. The changes have dramatically increased the utilization of services at public health facilities.

For private teaching hospitals, Punjab has the largest caseload, followed by Sindh and KPK. (As mentioned earlier, there is no private teaching hospital in Balochistan.) Similarly the mean caseload for private large hospitals was greatest for Punjab, followed by Sindh and Balochistan. (No private large hospital in KPK was available for sampling.)Among medium-sized private hospitals, the largest average caseload was in KPK, while the provinces of Sindh and Punjab treated less than half as many PAC cases in this type of health facility. The mean numbers of cases treated annually insmall private hospitals were largest in Punjab, followed by Sindh, KPK and Balochistan.

\section{4: Treatment forpost-abortion complications at Public- sector facilities between 2002-2012}

Next, we compare the estimated numbers of post-abortion complication cases treated in public facilities in 2002 and in 2012. Estimatesfor the private sector in 2002 were small and do not permit change analysis across the two time periods.

The annual number of patients with post-abortion complications treated at public health facilities increased moderately from 246,000to 267,000 in 2012 (Table 5.3). In view of the increase in Pakistan's population size during the last decade, this public-sector increase in PAC cases is a very modest one. The number grew rapidly in Sindh (from 57,200 PAC cases to 74,000), it doubled in

Balochistan (from 15,000 to 31,700 ), remained virtually unchanged in Punjab(126,200 to 126,800 ), and fell in KPK (from 47,500 to 34,700 ). 
Table 5.3: Annual number of post-abortion complication (out and in-patients) cases treated in public health facilities, by province, Health Facilities Survey, 2002 and 2012

\begin{tabular}{|c|c|c|c|c|c|c|c|c|c|c|}
\hline \multirow[b]{3}{*}{ Annual no. } & \multicolumn{10}{|c|}{ Province } \\
\hline & \multicolumn{2}{|c|}{ Punjab } & \multicolumn{2}{|c|}{ Sindh } & \multicolumn{2}{|c|}{ KPK } & \multicolumn{2}{|c|}{ Balochistan } & \multicolumn{2}{|c|}{ Total } \\
\hline & 2002 & 2012 & 2002 & 2012 & 2002 & 2012 & 2002 & 2012 & 2002 & 2012 \\
\hline Out-patients & 80,866 & 80,213 & 31,910 & 53,781 & 31,846 & 13,924 & 12,660 & 23,383 & 157,282 & 171,301 \\
\hline In-patients & 45,400 & 46,576 & 25,312 & 19,731 & 15,734 & 20,791 & 2,480 & 8,275 & 88,926 & 95,373 \\
\hline Out and in-patients & 126,267 & 126,789 & 57,222 & 73,512 & 47,580 & 34,715 & 15,140 & 31,659 & 246,208 & 266,675 \\
\hline
\end{tabular}

Source: Health Facilities Survey, weighted results.

Overall, there has been an increase in the number of cases treated as out-patients at public health facilities-from 157,000 in 2002 to 171,000 in 2012-as well as a moderate increase in the number of in-patients-from 89,000 to 95,000 . Sindh(32,000 to 54,000 out-patients)and Balochistan show an increase in out-patients, whereas KPK shows a decrease. There has been essentially no change in outpatient and inpatient numbersin Punjab's public sector. Sind shows a decline in the number of in-patients served, whereasBalochistan and KPK show an increase (Table 5.3).

An increase in the proportion of outpatient cases and a corresponding decrease in inpatient ones are suggestive of a decrease in the number of women seeking induced abortions who experience severe complications, possibly as a result of their growing use of relatively safer methods of abortion.Improvements in the quality of PAC services could be another possible reason for this change in Sindh province. The decline in abortion-related complication cases in the public health sector of KPK could be due to the decreased availability of medical staff to work inPAC-related services as a result of the serious security situation in that province.

\section{Caseloadchanges by type of public facility}

In this section we analyse changes in average caseload by province and by level of public facility. Figure 5.3 shows that at the national level, the mean number of PAC cases in public health facilities dropped slightly, from 317 to 289 . Declines were seen in the Punjab and especially in KPK, while the mean caseload of PAC cases increased in Sindh and Balochistan. When these changes are disaggregated at the facility level, (Figure 5.4) a pattern emerges of caseloads beinglargest at the public teaching hospitals, of intermediate size at DHQs and smallest in RHCs. 
Figure 5.3: Mean annual number of PAC cases per public health facility, by province, Health Facilities Survey, 2002 and 2012

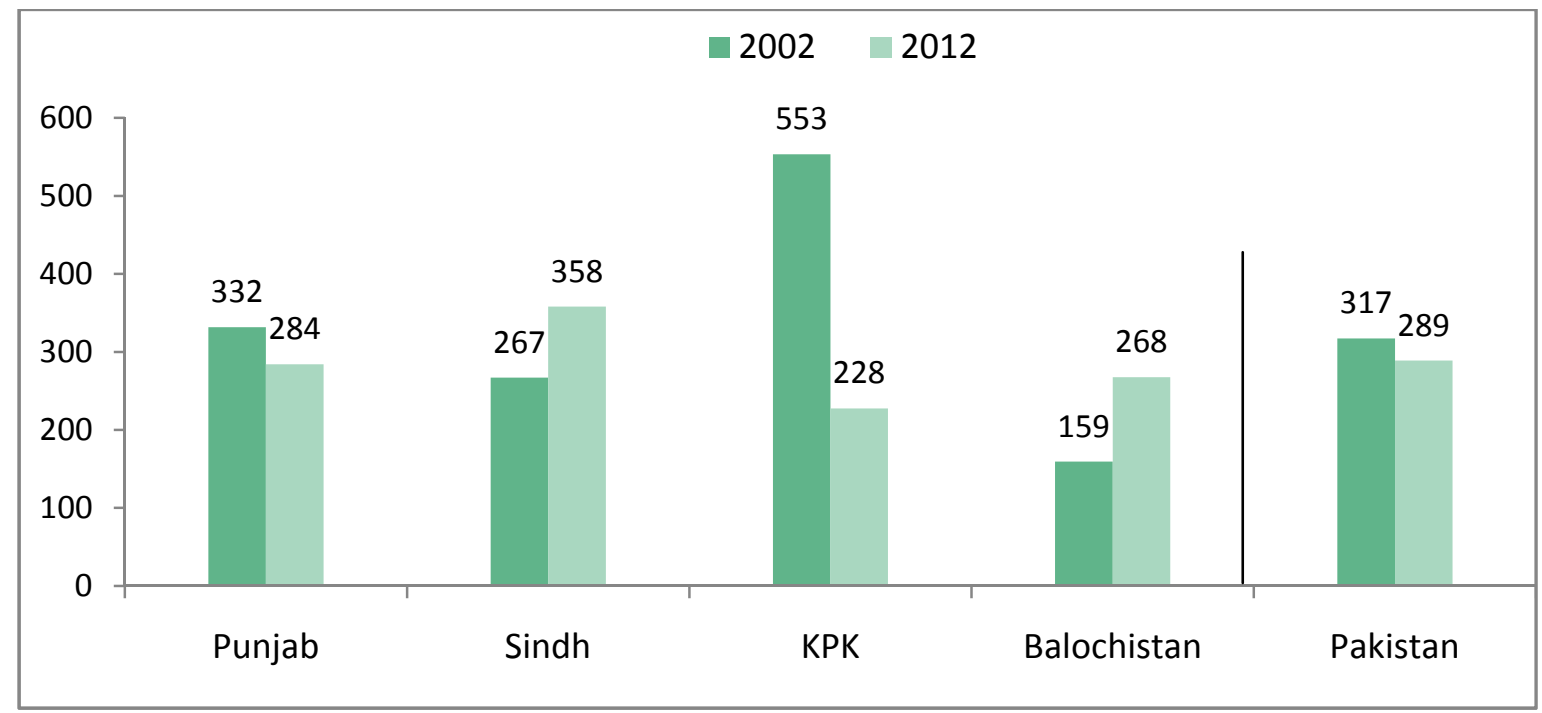

Source: Health Facilities Survey, weighted results.

Figure 5.4: Mean annual number of out-and in-patient PAC cases in public-sector facilities, by type of facility, Health Facilities Survey, 2002 and 2012

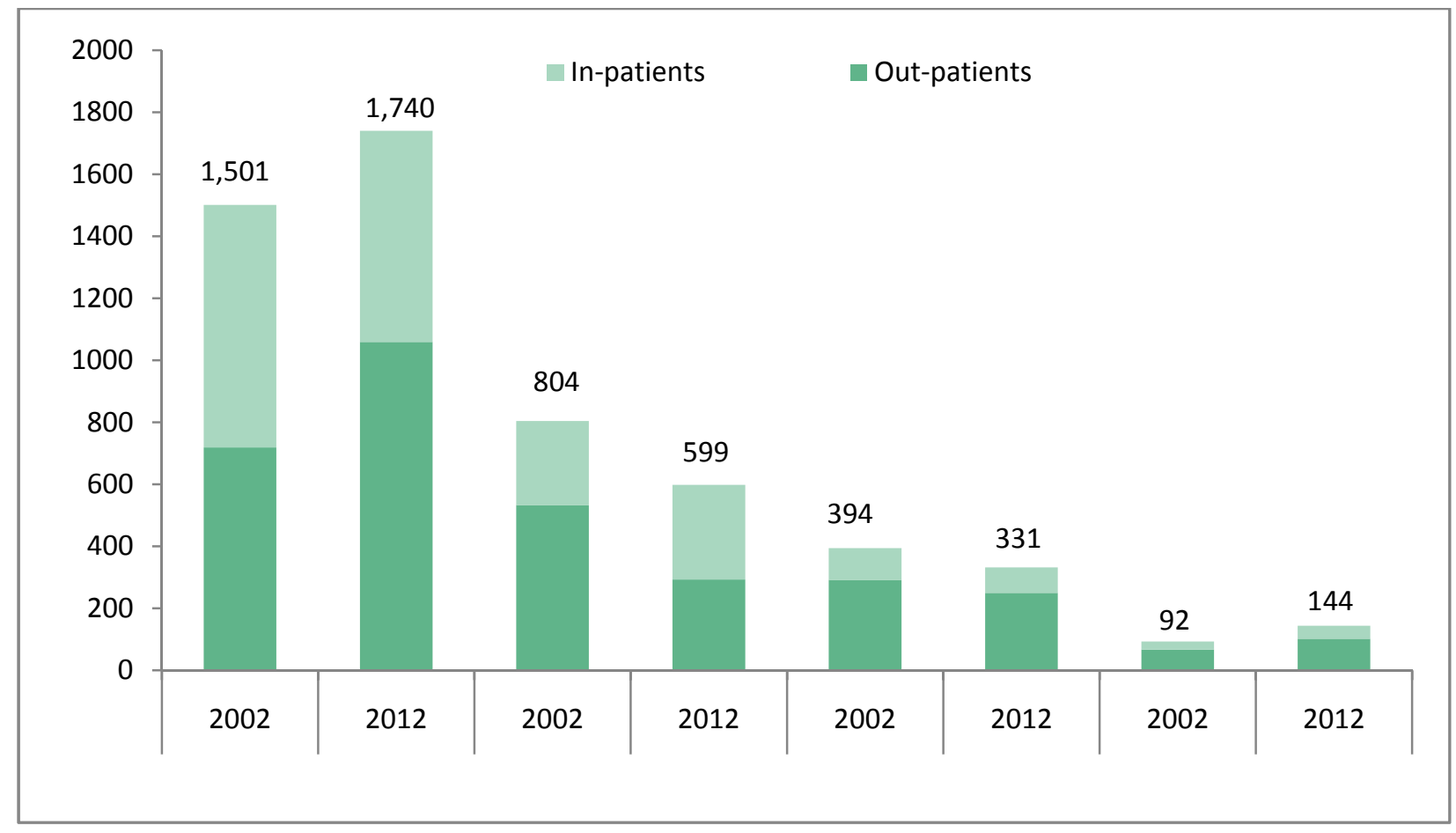

Source: Health Facilities Survey, weighted results.

The reason for this pattern is probably because most public-sector teaching and DHQ hospitals are large hospitals, while THQs and RHCs are small facilities. Therefore, large hospitals will always have larger caseloads, and vice a versa. 
Table 5.4: Mean annual number* of public-sector PAC (outand in-patients) cases, by type of facility and by province, Health Facilities Survey, 2002 and 2012

\begin{tabular}{|c|c|c|c|c|c|c|c|c|c|c|}
\hline \multirow[b]{3}{*}{ Mean annual number: } & \multicolumn{10}{|c|}{ Type of facility } \\
\hline & \multicolumn{2}{|c|}{ Teaching } & \multicolumn{2}{|c|}{ DHQ } & \multicolumn{2}{|c|}{ THQ } & \multicolumn{2}{|c|}{ RHC } & \multicolumn{2}{|c|}{ Total } \\
\hline & 2002 & 2012 & 2002 & 2012 & 2002 & 2012 & 2002 & 2012 & 2002 & 2012 \\
\hline Punjab & & & & & & & & & 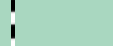 & \\
\hline Out-patients per facility & 631 & 700 & 554 & 298 & 427 & 333 & 79 & 103 & 212 & 180 \\
\hline In-patients per facility & 651 & 596 & 366 & 311 & 100 & 119 & 36 & 51 & 119 & 104 \\
\hline Out and in-patients per facility & 1,281 & 1,296 & 920 & 610 & 527 & 452 & 115 & 155 & 332 & 284 \\
\hline$\%$ out-patients & 49 & 54 & 60 & 49 & 81 & 74 & 69 & 67 & 64 & 63 \\
\hline Sindh & & & & & & & & & I & \\
\hline Out-patients per facility & 463 & 2,201 & 662 & 501 & 190 & 188 & 60 & 141 & 149 & 262 \\
\hline In-patients per facility & 915 & 856 & 386 & 252 & 160 & 79 & 23 & 40 & 118 & 96 \\
\hline Out and in-patients per facility & 1,379 & 3,057 & 1,048 & 753 & 351 & 267 & 83 & 181 & 267 & 358 \\
\hline$\%$ out-patients & 34 & 72 & 63 & 67 & 54 & 70 & 72 & 78 & 56 & 73 \\
\hline KPK & & & & & & & & & i & \\
\hline Out-patients per facility & 1,146 & 587 & 765 & 120 & 37 & 71 & 26 & 60 & 370 & 91 \\
\hline In-patients per facility & 1,136 & 843 & 272 & 642 & 31 & 10 & 0 & 1 & 183 & 136 \\
\hline Out and in-patients per facility & 2,282 & 1,429 & 1,037 & 762 & 68 & 81 & 26 & 61 & 553 & 228 \\
\hline$\%$ out-patients & 50 & 41 & 74 & 16 & 54 & 88 & 100 & 98 & 67 & 40 \\
\hline Balochistan & & & & & & & & & ! & \\
\hline Out-patients per facility & 1,920 & 576 & 75 & 336 & 381 & 470 & 54 & 75 & 133 & 198 \\
\hline In-patients per facility & 852 & 228 & 36 & 100 & 0 & 32 & 0 & 54 & 26 & 70 \\
\hline Out and in-patients per facility & 2,772 & 804 & 111 & 436 & 381 & 502 & 54 & 129 & 159 & 268 \\
\hline$\%$ out-patients & 69 & 72 & 68 & 77 & 100 & 94 & 100 & 58 & 84 & 74 \\
\hline Pakistan & & & & & & & & & ! & \\
\hline Out-patients per facility & 719 & 1,059 & 533 & 293 & 292 & 250 & 67 & 102 & 203 & 186 \\
\hline In-patients per facility & 782 & 681 & 271 & 305 & 102 & 82 & 26 & 42 & 115 & 103 \\
\hline Out- and in-patients per facility & 1,501 & 1,740 & 804 & 599 & 394 & 331 & 92 & 144 & 317 & 289 \\
\hline$\%$ out-patients & 48 & 61 & 66 & 49 & 74 & 76 & 73 & 71 & 64 & 64 \\
\hline No. of facilities & (40) & (40) & (33) & (42) & (24) & (31) & (44) & (21) & (63) & (134) \\
\hline
\end{tabular}

Source: Health Facilities Survey, weighted results.

* Mean of average per year and past-year estimates.

The proportion of clients treated as outpatients (Table 5.4) increased for public teaching hospitals whereas it fell for DHQs in the period 2002-2012. There was essentially no change in the proportion of outpatients in THQs whereas there was a small increase in RHCs. An increase in the proportion of total cases that are outpatients and a corresponding decline in the proportion of inpatients may be reflecting a reduction in the severity of complications. It could also reflect the health facilities' use of safer and less invasive methods such as MVA/EVA andmisoprostol, which are manageable as outpatient procedures. 


\section{Rates of treatment for post-abortion complications}

Table 5.5 shows the number of women treated for post-abortion complications per 1,000 women aged 15-49 years in 2012. In Pakistan, approximately 15 women out of every 1,000 women of reproductive age sought treatment for post-abortion complications. Balochistan province had the highest rate of 20 per 1,000, followed by Sind and Punjab with almost similar rates of around 16 per 1,000 . The lowest rate was in KPK-9 per 1,000. While a large proportion of these treatments would be for complications from induced abortion complications, some were for spontaneous pregnancy losses (perhaps about 20-25\% of all cases, based on estimates for 2002).

Table 5.5: Number and rate of women treated for post-abortion complications per 1,000 women aged 15-49 in 2012, by province, Health Facilities Survey, 2012

\begin{tabular}{lrrr} 
Pakistan & $\begin{array}{c}\text { No. of women age 15-49 } \\
\text { in 2012* }\end{array}$ & $\begin{array}{r}\text { No of women treated for } \\
\text { abortion-related complication } \\
\text { (HFS data) }\end{array}$ & $\begin{array}{r}\text { Rate per 1,000 women } \\
15-49\end{array}$ \\
Punjab & $46,129,789$ & 695,861 & 15 \\
Sindh & $26,315,742$ & 416,433 & 16 \\
KPK & $10,919,202$ & 174,908 & 16 \\
Balochistan & $6,545,611$ & 57,159 & 9 \\
\hline
\end{tabular}

Source: Health Facilities Survey, weighted results.

*Population projections for 2012 by the Population Council.

If we look at the rate of women treated for abortion related complications in public-sector facilities we see that the rates dropped slightly from 7 to 6 per 1,000 women between 2002 and 2012. The decline in the rate of PAC treatment in the public sector over the past decade is largest for KPK province, which dropped by almost half. In contrast, the rate for Balochistan increased.

Comparing the overall rate of 15 shown in Table 5.5 and the estimated rate of 6 for the public sector in Table 5.6 suggests that the private sector accounts for a rate of 9 per 1,000 women in 2012 (15 per 1,000 minus 6 per 1,000). While this needs further exploration, this may be due to "unusual security risks faced by women in KPK in travelling to health centres" in the last few years. The differentialalso supports the hypothesis that post-abortion care may be increasingly diverted to private-sector facilities, which are likely to treating women suffering from less severe complications. 
Table 5.6: Rate of women treated for abortion complications in public-sector health facilities per 1,000 women 15-49, by province, Health Facilities Survey, 2002 and 2012

\begin{tabular}{|c|c|c|c|c|c|c|}
\hline & \multicolumn{2}{|c|}{ No. of women age $15-49 *$} & \multicolumn{2}{|c|}{$\begin{array}{l}\text { No. of women treated in public } \\
\text { facilities for abortion-related } \\
\text { complications (HFS data) }\end{array}$} & \multicolumn{2}{|c|}{ Rate per 1,000 women $15-49$} \\
\hline & 2002 & 2012 & 2002 & 2012 & 2002 & 2012 \\
\hline Pakistan & $33,618,228$ & $46,129,789$ & 246,208 & 266,675 & 7.3 & 5.8 \\
\hline Punjab & $18,855,022$ & $26,315,742$ & 126,267 & 126,789 & 6.7 & 4.8 \\
\hline Sindh & $7,708,273$ & $10,919,202$ & 57,222 & 73,512 & 7.4 & 6.7 \\
\hline KPK & $4,454,524$ & $6,545,611$ & 47,580 & 34,715 & 10.7 & 5.3 \\
\hline Balochistan & $1,571,405$ & $2,331,199$ & 15,140 & 31,659 & 9.6 & 13.6 \\
\hline
\end{tabular}

Source: Health Facilities Survey, weighted results.

*Population projections for 2012 by the Population Council.

Comparison of the 2002 rate of facility-based treatment of abortion complications ( 7 per 1,000 women) with the rate for 2012 (15 per 1,000) appears to indicate that the rate increased. However, the rates are not directly comparable because the private sector was not measured in 2002 and it is covered in 2012. In addition, some of the apparent increase in the rate probably reflects growing access to health care services in the private sector and the expansion of the private health sector itself, which may be picking up women who may not have obtained treatment a decade ago. In addition, it likely also partly reflects a shift towards the use of misoprostol to terminate unwanted pregnancies. Many women who do not use this method correctly because they lack information will be forced to seek medical treatment for an incomplete abortion or prolonged heavy bleeding; because the provider of the drug has told them to go to a clinic soon after bleeding starts; or because they themselves are concerned about waiting for the bleeding to end. It is possible that a part of the difference in rates between the two years may be a true increase, reflecting an increase in women's recourse to abortion, but further analyses are needed to determine this. 



\section{Chapter 6 Family Planning Counselling and Services: An Important Aspect of Post-Abortion Care}

Women seeking induced abortions are likely to have an unmet need for effective family planning. Offering women family planning counselling and services after they have had an abortion or received post-abortion care (PAC) makes eminent sense and is a well-established means of reducing future unintended pregnancies and the need for repeat or unsafe abortions ${ }^{38}$. Contraceptive counselling and services are essential elements of PAC management, and providers must be adequately trained to offer such services, given that a woman in this situation is likely to be under emotional and physical stress. She needs support and guidance and, most importantly, she needs to avoid another unwanted, unplanned or mistimed pregnancy.

This chapter deals with the provision of family planning counselling and contraceptive services in health facilities providing PAC.

\section{1: Contraceptive counselling}

Respondents to the Health Facilities Survey (HFS) were asked whethertheir institution provided post-abortioncounselling on the use of contraception. Respondents of about three-quarters of public and private teaching hospitals and of District Headquarters Hospitals, two-thirds of Tehsil Headquarters Hospitalsand about one-half of Rural Health Centresreported that they provide counselling on the use of contraceptives (Figure 6.1). 
Figure 6.1: Proportion of public and private-sector health facilities providing post-abortion counselling on birth spacing and contraception, by type of facility, Health Facilities Survey, 2012

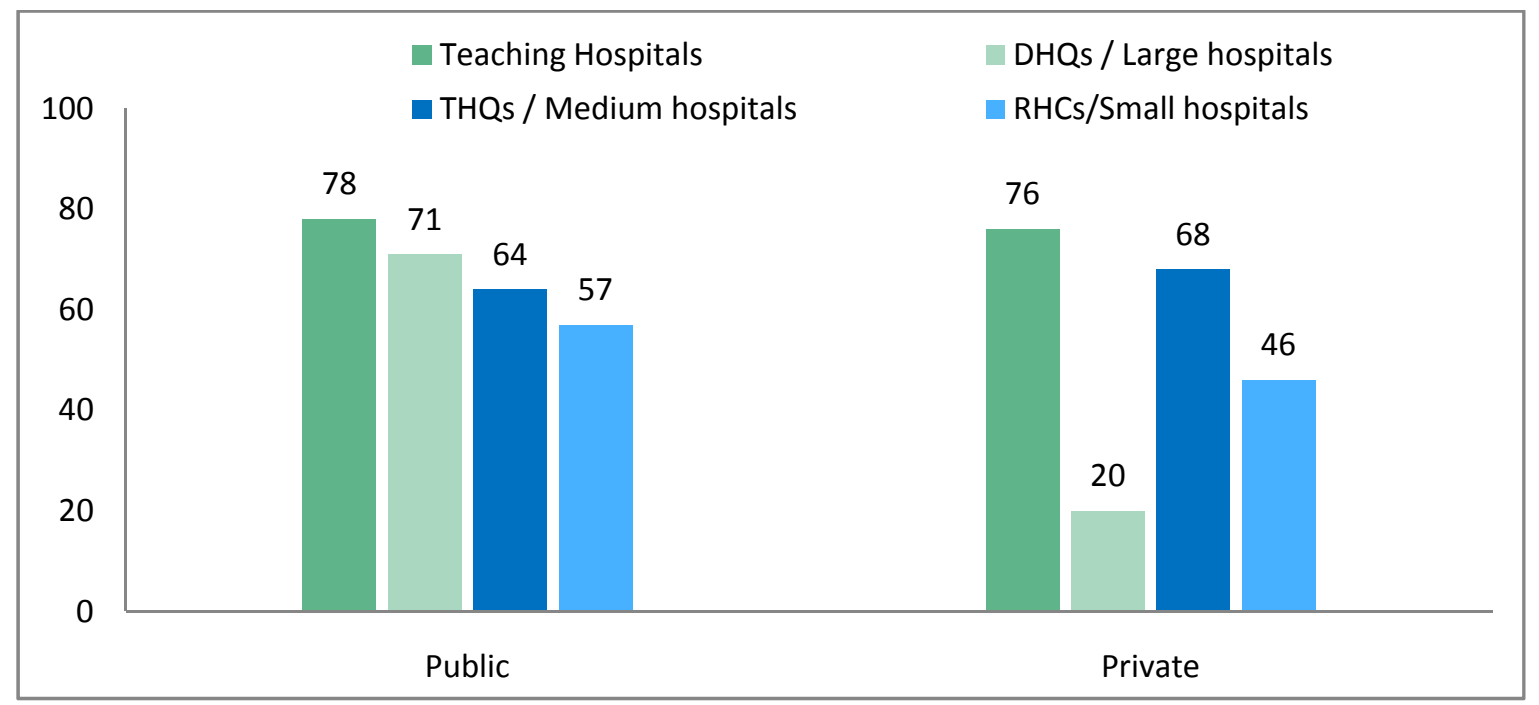

Source: Health Facilities Survey, weighted results.

A substantial proportion of all categories of public-sector facilities and most categories of privatesector facilities offer contraceptive counselling to PAC patients. One exception is private large hospitals-only $20 \%$ of which offered this service. However, there is a substantial remaining gap: One-fifth to one-third of other categories of large and medium-sized facilities do not offer such counselling, and this gap is larger among the smallest facilities.

Figure 6.2illustrates the proportion of women perceived by the medical staff at the health facilities to have receivedpost-abortion counselling on any of a large number of health-related topics (family planning, medications, nutrition, follow-up visits, personal hygiene, etc.) before leaving the health facility. Overall, more than $85 \%$ of women receiving PAC in both public and private facilities are reported to havebeen given counselling on such matters. Although the differencesare small, proportionately more women in private than in public hospitals receive broad counselling of this type. 
Figure 6.2: Proportion of women treated in health facilities who receive post-abortion counselling on one or more of a range of health-related issues, ${ }^{*}$ by sector and type of facility, Health Facilities Survey, 2012
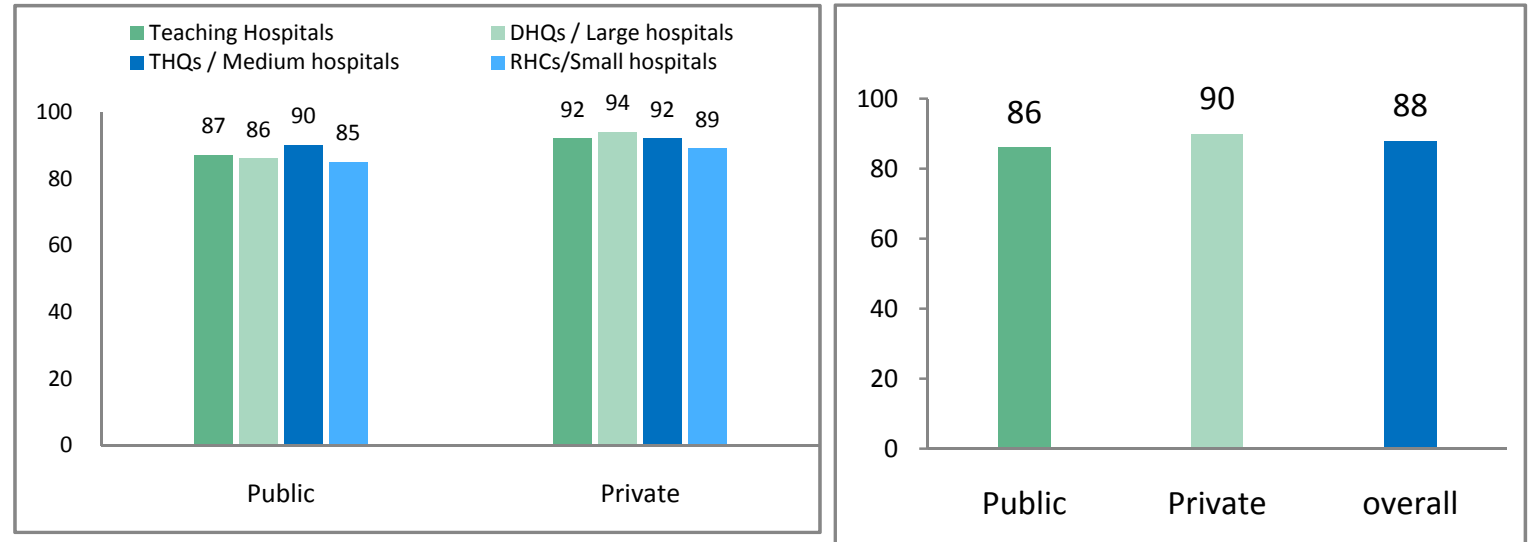

Source: Health Facilities Survey, weighted results.

* Including, family planning, nutrition, hygiene, follow-up visits, medications, etc.

Results from the 2002 and 2012 surveys of health professionals (a broad cross-section of experts, separate from the health facility survey) show that the proportion of respondents who thought that women should be given family planning counselling wasmuch higher in 2012 than 2002 (61\% vs. $36 \%$, data not shown).

When asked about the adequacy of the counselling services currently being provided, only $50 \%$ of HPS respondents were of the view that post-abortion counselling on birth spacing/contraception was adequate (Table 6.1).

Table 6.1: Proportion of health professionals who consider post-abortion counselling on selected topics to be adequate, Health Professionals Survey, 2012

Counselling topic

Causes of abortion

Medication

Proper nutrition/rest

Follow-up appointments

Return to fertility

Resumption of sex

Counselling of husband about wife's medical condition

Birth spacing/contraception

Personal hygiene

Others

\section{Percent}

50

48

57

45

37

20

50

49

7

(102)

No. of respondents

Source: Health Professionals Survey. 
About one-half thought that counselling on medications, nutrition, personal hygiene and follow up visits was adequately provided,but only $37 \%$ found counselling on the causes of abortion and the resumption of sex to be adequate. According to these key informants, the quality of the postabortion counselling being offered needs much improvement.

\section{2: Contraceptive services}

There is a wealth of evidence demonstrating the obvious association between low rates of contraceptive use and high levels of unplanned pregnancy ${ }^{39,40}$. Increasing the level of contraceptive use dramatically reduces abortion rates, since most induced abortions are in response to an unplanned pregnancy ${ }^{41,42}$.

The overall proportion of facilities that reported commonly offering contraceptive methods to post-abortion patients is quite high (Table 6.2). In both health sectors, teaching hospitals and the smallest facilities are more likelyor equally likely to provide PAC patients withreversible contraceptivemethods (such as IUCDs, injectables, the pill or the condom), compared to large and medium-sized hospitals. The teaching and larger hospitals, which are likely to have on staff doctors who can provide permanent or long-term methods of contraception (Minilap and noscalpel vasectomy, for example) and to have more modern and better equipped facilities,are more geared towards providing these methods.

Table 6.2: Percent of facilities reporting that they commonly offer specific types of contraceptives to post-abortion patients, by type of health facility, Health Facilities Survey, 2012

\begin{tabular}{|c|c|c|c|c|c|c|c|c|c|c|c|}
\hline \multirow{3}{*}{$\begin{array}{l}\text { Contraceptive } \\
\text { methods }\end{array}$} & \multicolumn{4}{|c|}{ Public } & \multicolumn{4}{|c|}{ Private } & \multicolumn{2}{|c|}{ Overall } & \multirow{2}{*}{ Total } \\
\hline & Teaching & DHQ & THQ & RHC & Teaching & Large & Medium & Small & Public & Private & \\
\hline & $\%$ & $\%$ & $\%$ & $\%$ & $\%$ & $\%$ & $\%$ & $\%$ & $\%$ & $\%$ & $\%$ \\
\hline Pills & 88 & 76 & 81 & 93 & 95 & 60 & 59 & 85 & 89 & 79 & 83 \\
\hline Injectables & 88 & 78 & 84 & 93 & 95 & 60 & 59 & 85 & 89 & 79 & 83 \\
\hline Implants & 36 & 19 & 12 & 2 & 9 & 19 & 20 & 11 & 7 & 13 & 11 \\
\hline Female sterilization & 76 & 60 & 36 & 6 & 81 & 42 & 45 & 33 & 20 & 37 & 31 \\
\hline IUCD & 82 & 82 & 84 & 83 & 95 & 60 & 75 & 77 & 83 & 77 & 79 \\
\hline Condom & 67 & 44 & 74 & 87 & 86 & 60 & 32 & 59 & 79 & 54 & 64 \\
\hline Vasectomy & 9 & 0 & 5 & 0 & 5 & 0 & 1 & 1 & 1 & 1 & 1 \\
\hline \multicolumn{12}{|c|}{ Proportion of women who leave the health facility with a contraceptive method: } \\
\hline Percent & 76 & 49 & 57 & 53 & 43 & 77 & 43 & 44 & 54 & 46 & 49 \\
\hline
\end{tabular}

Source: Health Facilities Survey, weighted results.

In public-sector facilities, three-fourths of PAC cases in teaching hospitals and about one-half in DHQs, THQs and RHCs leave the facility with a contraceptive method. In the private sector, with the exception of private large hospitals, the proportion of patients leaving with a contraceptive method is lower. These findings again suggest that public-sector facilities are better equipped 
than private hospitals to provide PAC patients with family planning services. Nevertheless, even in the public sector only just over half of all PAC clients leave the facility with a contraceptive method $54 \%$ ) and in the private sector less than half do so (46\%).

\section{Referral of clients for contraceptive services}

Respondents in all facilities were asked whether they refer clients elsewhere to obtain contraceptive services. Ninety-four percent of DHQs, $88 \%$ of teaching hospitals, $86 \%$ of RHCs and $76 \%$ of THQs report doing so (Table 6.3).

Table 6.3: Percent of facilities that refer clients to other health institutions, by type of facility, Health Facilities Survey, 2012

\begin{tabular}{|c|c|c|c|c|c|c|c|c|c|}
\hline & \multicolumn{4}{|c|}{ Public } & \multicolumn{4}{|c|}{ Private } & \multirow[b]{2}{*}{ Total } \\
\hline & Teaching & DHQ & THQ & RHC & Teaching & Large & Medium & Small & \\
\hline & $\%$ & $\%$ & $\%$ & $\%$ & $\%$ & $\%$ & $\%$ & $\%$ & $\%$ \\
\hline Percent that refer to: & 88 & 94 & 76 & 86 & 85 & 80 & 77 & 59 & 72 \\
\hline RHS-A Centre & 96 & 61 & 53 & 18 & 70 & 0 & 20 & 40 & 33 \\
\hline FWC & 0 & 14 & 9 & 2 & 12 & 0 & 0 & 5 & 4 \\
\hline Private clinics/Hospital & 0 & 14 & 9 & 8 & 7 & 0 & 16 & 11 & 10 \\
\hline $\mathrm{RHC}$ & 0 & 0 & 0 & 2 & 0 & 0 & 0 & 2 & 2 \\
\hline THQ & 0 & 13 & 0 & 23 & 0 & 0 & 0 & 7 & 10 \\
\hline $\mathrm{DHQ}$ & 0 & 0 & 29 & 46 & 6 & 100 & 64 & 48 & 45 \\
\hline LHW & 0 & 0 & 6 & 2 & 0 & 0 & 0 & 0 & 1 \\
\hline Medical store & 3 & 0 & 3 & 2 & 11 & 0 & 0 & 3 & 2 \\
\hline Others & 4 & 13 & 9 & 16 & 24 & 0 & 0 & 11 & 11 \\
\hline
\end{tabular}

Source: Health Facilities Survey, weighted results.

Note: Based on multiple responses.

The locations to which women are most likely to be referredare specialized family planning service centres (RHS-A) attached to teaching hospitals and DHQs. RHCs, on the other hand, are more likely to refer patients to DHQs first and to RHS-A centres second. In the private sector, $70 \%$ of teaching hospitals refer clients to RHS-A centres, while the other types of facilities mostly refer women to a DHQ.Only small numbers of private facilities refer women to facilities that offer more comprehensive family planning services like RHS-A Centres and Family Welfare Centres of the Population Welfare Department, where a wide range of contraceptive choices is available.

Public health facilities are not likely to refer their PAC clients to FWCs or LHWs even thoughclinics staffed by this type of health worker are probably closer to the women's homes and communities, which would make it easier for themto getresupplies or obtain advice about possiblecontraceptive side effects. Similarly, very few teaching hospitals and other health facilities in the private sector refer clients to FWCs, and none refer them to LHWs. [This indicatesa lack of coordination for cross-referrals between Health Departments and Population Departments, or between public and private facilities. This is another important programmingopportunity lostsince 
focus group research shows that LHWs are one of the major providers of family planning in rural areas for poor women].

"We go to the LHW for any [FP] services that we require. We don't go to anyone else.

They are our neighbours as well so they help us in all matters. Whatever we need we take from the LHW"(IDI-housewife, 2 children, Hafizabad).

"If we face any problem we contact baji(LHW). She visits us at home and we discuss every problem with her. She counsels us on FP methods. If one method does not suit then she suggests a different method"(FGD-women, Khanewal).

\section{3: Provider attitudes to PAC, contraception and induced abortion}

The Health Facilities Survey (HFS) respondents were asked to describe their attitudes and values surrounding the issues of post-abortion care, contraception and induced abortion.Their responses more or less mirror those of the participants in the Health Professionals Survey (HPS), who were asked similar questions. Overall,a majority of HFS and HPS respondents agreed with the statement that post-abortion care should be more widely available and that PAC can save women's life (Figures 6.3 and 6.4).

Figure 6.3: Proportion of health facility respondents of who agree with various statements regarding post-abortion care, Health Facilities Survey, 2012

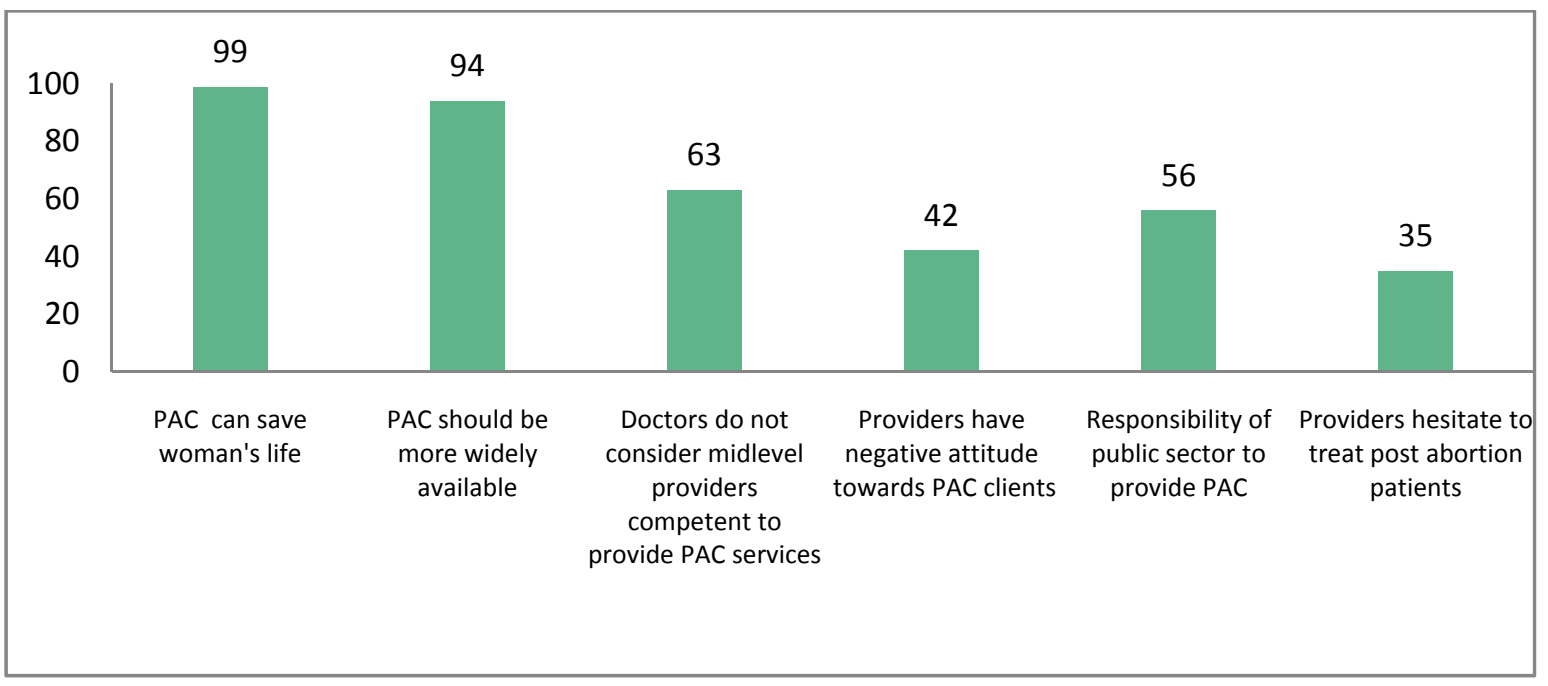

Source: Health Facilities Survey, weighted results. 
Figure 6.4: Proportion of health professionals who agree with various statements regarding post-abortion care, Health Professionals Survey, 2012

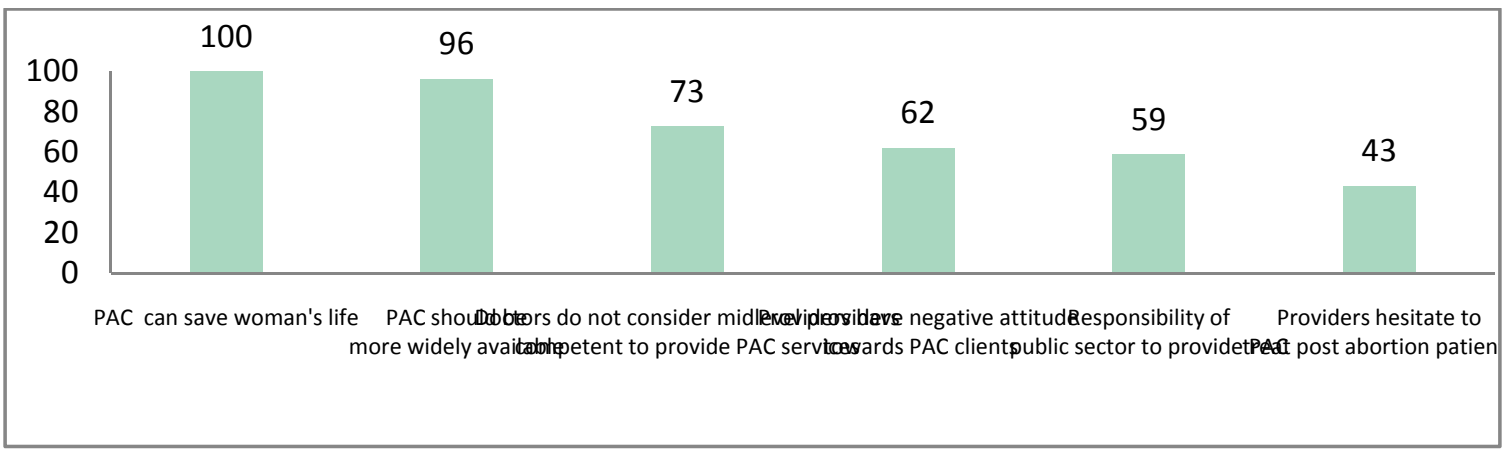

Source: Health Professionals Survey.

Slightly less than two-thirds of the HFS respondents and three-quarters of the HPS respondents agreed that doctors do not consider paramedics competent enough to provide PAC services. This is an important finding because, as we saw in Chapter 4, LHVs/Nurses/Midwivesare available at almost all public and private health facilities whereas there is incomplete coverage by doctors and gynaecologists in both sectors. If not considered competent, they are unlikely to be able to develop the necessary skills to offer this type of reproductive health care, and might be hesitant in or restricted from providing PAC services.

Over one-half of the health respondents in the two surveys agreed with the statement that it is the responsibility of the public sector to provide PAC. Slightly less than two-thirds of those taking part in the HPS and $42 \%$ of the HFS respondents agreed that providers have a negative attitude towards PAC clients, which suggest a strong bias on the part of many providers against women seeking post-abortion care. Moreover, one-third of the HFS respondents and $43 \%$ of those participating in the HPS were of the opinion that providers are generally reluctant to treat patients with post-abortion complications. These responses further strengthen the widespread belief that PAC seekers face varying levels of discriminationon the part of their caregivers.

There was universal agreement among both groups of respondents that induced abortion is an acceptable practice if the foetus is abnormal, or to protect a woman's life (Figures 6.5 and 6.6).

Figure 6.5: Proportion of health facility respondents who agree with various attitudes about the practice of abortion, Health Facilities Survey, 2012

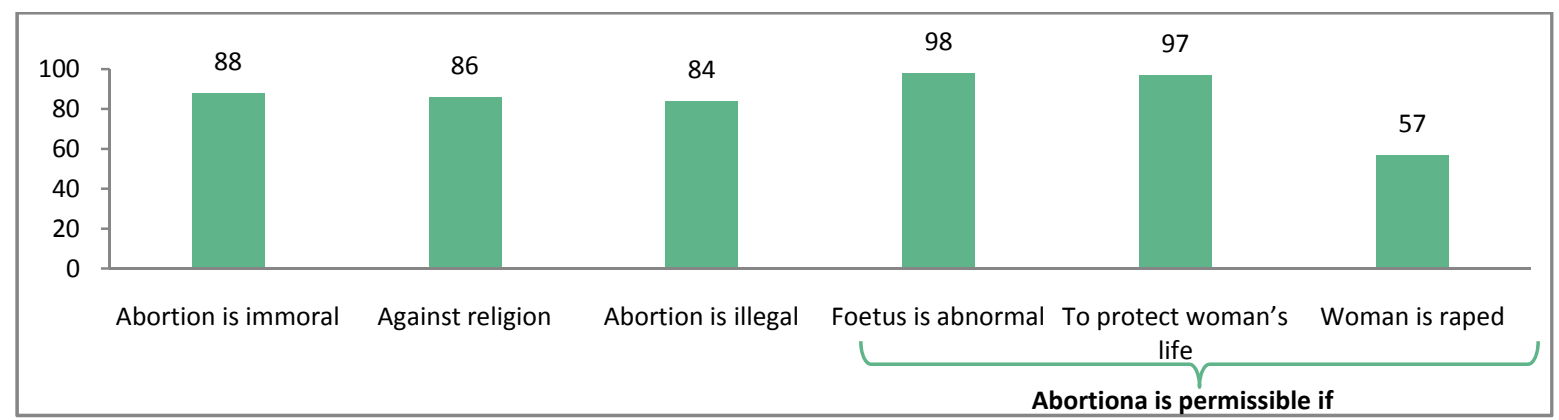

Source: Health Facilities Survey, weighted results. 
Figure 6.6: Proportion of health professionals who agree withvarious attitudes about the practice of abortion, Health Professionals Survey, 2012

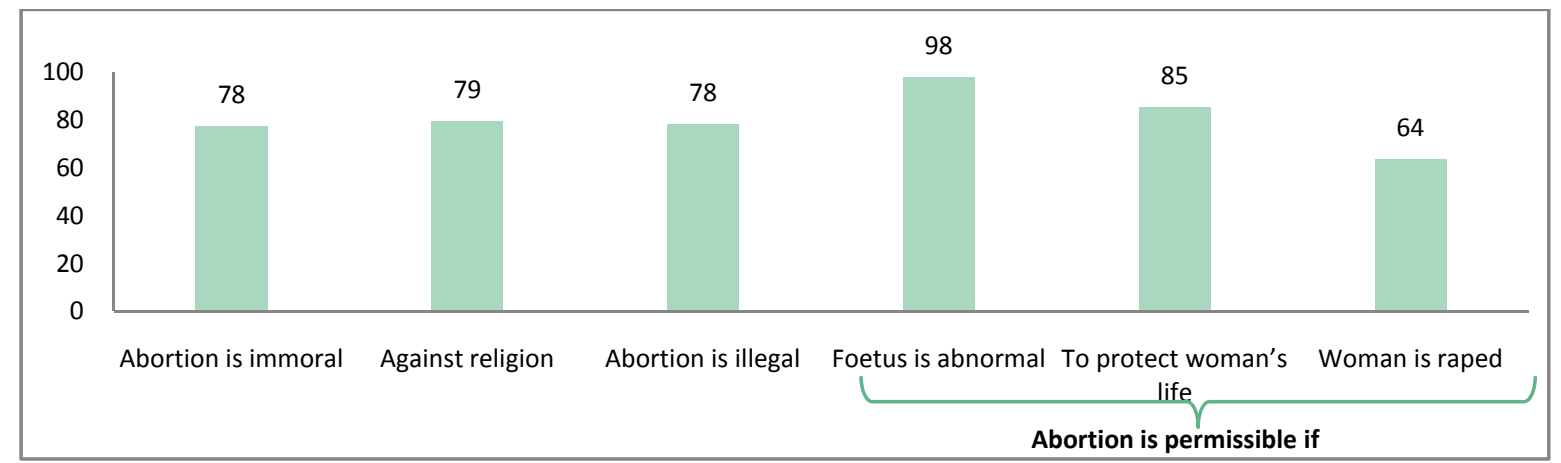

Source: Health Professionals Survey.

However, in response to the statement, 'Abortion is acceptable when a woman is raped,' $43 \%$ of HFS respondents and $36 \%$ of HPS participantsdisagreed. The majority of respondents in both surveys agreed that abortion is immoral, contrary to religion and illegal. However, there was some variation in these positionsaccording to the type of health worker (Figure 6.7).

Figure 6.7: Attitudes toward the practice of induced abortion, by type of health professional, Health Professionals Survey, 2012

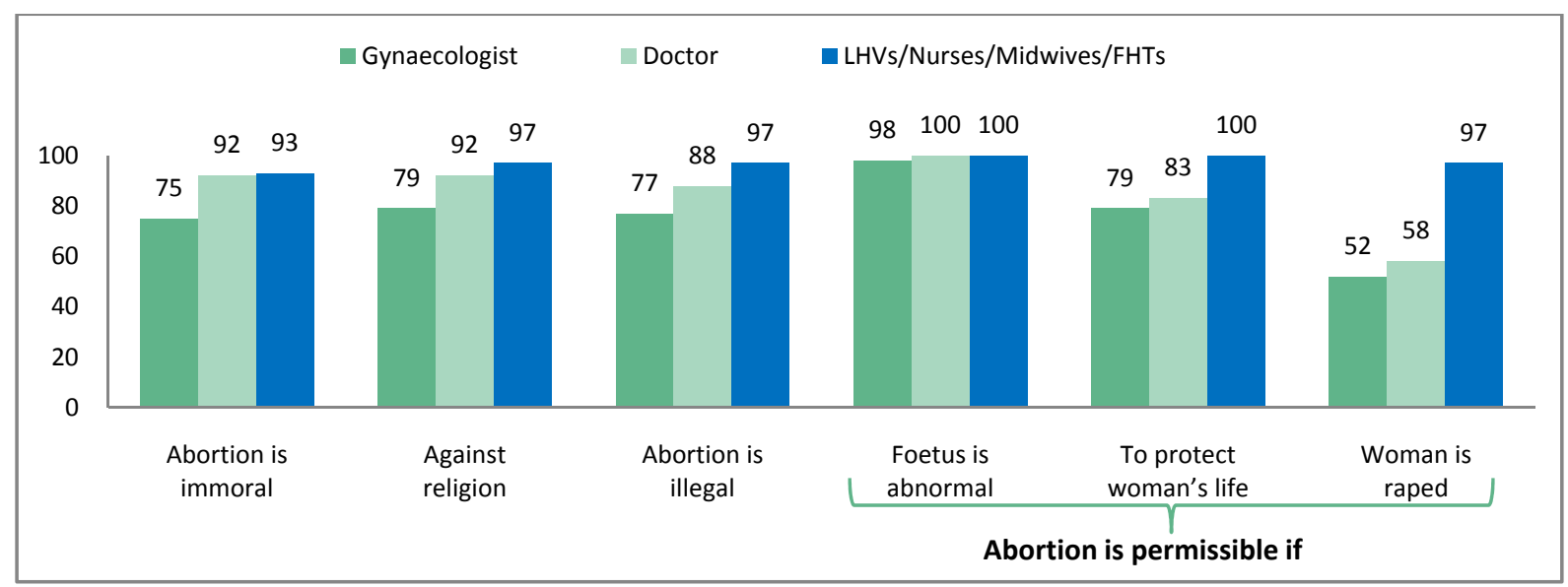

Source: Health Professionals Survey.

Almost all female paramedics (LHVs/Nurses/Midwives/FHTs) agreed that abortion is permissible if a woman has been raped compared to slightly more than one-half of the gynaecologists and doctors. But a higher proportion of LHVs/Nurses/Midwives than of doctors and gynaecologists believed that abortion is immoral, illegal and contrary to religion. 


\section{Chapter 7 Barriers in Accessing Post-abortion Care: Women, Men and Providers' Views}

\section{1: Introduction}

This chapter presents the findings of qualitative research comprising focus group interviews with women and in-depth interviews with women and providers, as well as informal discussions with men. The interviews present insights into the experiences and management of post-abortion complicationsas expressed by community voices. We especially focus on the various barriers that may delay or hinder seeking care when complications occur after an abortion. Coming a decade after the first national study on unwanted pregnancies and post-abortion complications, ${ }^{6}$ this research also provides a chance to compare the situation over the years.

Little research has been done onthe barriers which women face when accessing post-abortion care in Pakistan. This qualitative research aims to fill this gap in this area of women's reproductive health in Pakistan. In addition to this chapter, a short brief highlighting these issues of gender and poverty has also been prepared as part of the study. We plan to fully analyse the large amount of rich data that has emerged from the qualitative research in future work. In this chapter we are presenting the main findings of the study, especially as they relate to issues of barriers of access and information on post-abortion care.

\section{2: Objectives}

The objectives of the qualitative research were to capture women, men and local providers' voices on the demand side of abortion and post-abortion care by gathering information on barriers to seeking care in well-served and under-served areas, as well as the quality of abortion and post-abortion care available (mainly in rural areas of Pakistan). The research questions include:

- What abortion methods are poor and rural women using? How safe or unsafe are these methods?

- What are the main barriers that women face when they access abortion-related health care?

- Do poor and rural women report being able to access PAC if they face complications? 
- What are the main barriers faced by women in their households and communities that prevent them from accessing timely health care, and any additional issues related to postabortion care?

- What do women who seek PAC services perceive to be the attitudes of providers towards them?

- Are women given family planning counselling and services as part of their post-abortion care?

\section{3: Study methodology}

The study's main focuswas poor and rural women, since these are the groups who are likely to face the greatest health consequences of unintended pregnancy, unsafe abortion and the need for post- abortion care. Four poor urban and 11 rural communities were included to compare access issues faced by poor women in these different settings. Further, within rural settings we tried to capture differences between 'served' communities, those that were at least served by the government's Lady Health Worker (LHW) program (and may additionally have had other public and private facilities offering reproductive health care services) and 'under-served' communities, those who were not only devoid of LHW coverage but also lack any kind of general and reproductive health care facilities or services.

\section{Data collection methods}

Three main data collection techniques were used: in-depth interviews (IDIs), focus group discussions (FGDs), and informal group discussions. Additionally, social mapsof each study site and community profiles were developed in order to capture a picture of the communities being studied. Details of main data collection techniquesare given below:

a) 44 In-depth interviews (IDIs) were conducted with women who had experienced an induced abortion in the six months preceding the study in LHW-served communities. The rationale was to obtain information about the most commonly used methods of abortion, and the availability and quality of PAC services. Since no abortion case came up in non-LHW areas, no IDI was conducted there.

b) In-depth interviews with 19 community levelservice providers including LHWs, LHVs and TBAs or dais were conducted to supplement the findings from women. These providers are most likely to be aware of the obstacles that women face in receiving care in the selected primarily rural and poor communities.

c) Ten Focus group discussions (FGDs) were conducted with women with at least three living children both in LHW-served areas and under-served areas. It was assumed that these women 
were most likely to have had unwanted pregnancies and to be at high risk of unsafe induced abortion.

d)Ten Informal group discussions with currently married menin the LHW-served communities were also conducted. Keeping in view the important role of men in decision-making in the Pakistani context, their perspectives had to be included.

\section{Selection of communities}

The study was carried out in two of the more populous major provinces of Pakistan (Punjab and Sindh). The qualitative study was not conducted in KPK and Balochistan because of security reasons. FGDs and IDIs involved going to rural communities and asking questions about abortion and post-abortion care from women that would have been very risky under the prevailing security scenario.

In the first phase of fieldwork, carried out between January and March 2012, three rural and two urban districts were selected;all LHW served communities. The urban districts were Karachi in Sindh, and Rawalpindi in Punjab; the rural districts selected were Umerkot in Sindh and Hafizabad and Khanewal in Punjab.

As areas served by LHWs usually also have some public and even private health care and $\mathrm{RH}$ services, we decided that it was also necessary to add non-LHW areas to the sampling. If these areas were not included, we would be missing those women for whom seeking reproductive health services is all the more difficult because of a lack of services in their communities. Therefore, in a second phase, insights from women in under-servedareas were especially sought by inclusion of communities where there was no LHW-coverage.

We selected one district each from Punjab and Sindh among the 35 districts for which the Population Council had GIS Census data of health facilities (which allowed us to identify areas without LHWcoverage) ${ }^{*}$. Once the districts were selected (DG Khan in Punjab and Sanghar in Sindh) under-served areas within these districts were identified.

\section{Selection of respondents}

A combination of methods was used to select respondents for the IDIs. Communities within the selected districts were identified with support from provincial and district Health Departments. The district managers helped to identify Basic Health Units (BHUs) located in rural areas andin most areas, BHU staff, particularly LHWs, helped in identifying and recruiting the respondents. LHWs maintain a register of all married women of reproductive age within their catchment area

"GIS mapping of health and reproductive health facilities was carried out in 2007-2010 in 35 districts of Pakistan by Population Council. 
and know the current status of pregnancy, FP use and, at times, the abortion status of these women. In the selected served communities, LHWs identified women who had had an abortion in the last six months and obtained verbal consent for the interviews before the visit of the research team. In Karachi, community workers of a local NGO helped identify eligible respondents.

Participants of FGDs were also identified with the help of LHWs. In non-LHW communities, the research team recruited respondents for the FGDs themselves. In these under-served communities participants denied any practice of abortion in their communities. It was decided that the process of looking for eligible respondents for IDIs would be futile.

Community service providers (LHW, LHV and TBA) were identified bythe research team during the course of data collection in the community. Currently married men were recruited with the help of an influential male member of the community, who was usually identified by a LHW.

Table 7.1: District wise distribution of respondents by type of interview (IDIs, FGDs and informal discussions)

\begin{tabular}{|c|c|c|c|c|c|c|}
\hline \multirow[b]{2}{*}{ District } & \multirow[b]{2}{*}{$\begin{array}{l}\text { IDls with } \\
\text { women* }\end{array}$} & \multirow{2}{*}{$\begin{array}{l}\text { IDIs with } \\
\text { services } \\
\text { providers }\end{array}$} & \multicolumn{2}{|c|}{ FGDs with women } & \multicolumn{2}{|c|}{$\begin{array}{c}\text { Informal Discussions with } \\
\text { men }\end{array}$} \\
\hline & & & $\begin{array}{l}\text { No. of } \\
\text { FGDs }\end{array}$ & $\begin{array}{c}\text { No. of } \\
\text { participants }\end{array}$ & $\begin{array}{c}\text { No. of } \\
\text { discussions }\end{array}$ & $\begin{array}{c}\text { No. of } \\
\text { participants }\end{array}$ \\
\hline \multicolumn{7}{|c|}{ LHW-served areas } \\
\hline Rawalpindi & 8 & 4 & 1 & 11 & 1 & 6 \\
\hline Khanewal & 8 & 4 & 1 & 12 & 3 & 18 \\
\hline Hafizabad & 8 & 4 & 1 & 10 & 1 & 5 \\
\hline Karachi & 9 & 3 & 1 & 9 & 2 & 13 \\
\hline Umerkot & 11 & 4 & 2 & 23 & 3 & 17 \\
\hline \multicolumn{7}{|c|}{ Non-LHW areas } \\
\hline DG Khan & None & None & 2 & 18 & None & None \\
\hline Sanghar & None & None & 2 & 23 & None & None \\
\hline Total & 44 & 19 & 10 & 106 & 10 & 59 \\
\hline
\end{tabular}

*Women who had an abortion in the six months preceding the study 
Table 7.2: Socio-demographic profile of the respondents

\begin{tabular}{|c|c|c|c|c|c|c|}
\hline \multirow[b]{2}{*}{ Characteristics of respondents } & \multicolumn{2}{|c|}{ Women (IDIs) } & \multicolumn{2}{|c|}{ Women (FGDs) } & \multicolumn{2}{|c|}{ Service providers (IDIs) } \\
\hline & $\%$ & $\mathbf{N}$ & $\%$ & $\mathbf{N}$ & $\%$ & $\mathbf{N}$ \\
\hline \multicolumn{7}{|l|}{ Age (years) } \\
\hline $18-24$ & 4.5 & 2 & 4.9 & 5 & 0.0 & 0 \\
\hline $25-29$ & 25.0 & 11 & 19.6 & 20 & 21.1 & 4 \\
\hline $30-34$ & 36.4 & 16 & 27.5 & 28 & 15.7 & 3 \\
\hline $35-40$ & 18.2 & 8 & 25.5 & 26 & 21.1 & 4 \\
\hline $40+$ & 15.9 & 7 & 22.5 & 23 & 42.1 & 8 \\
\hline Total & 100.0 & 44 & 100.0 & $102 *$ & 100.0 & 19 \\
\hline \multicolumn{7}{|l|}{ Education of respondents } \\
\hline No schooling & 40.9 & 18 & 58.8 & 60 & 26.3 & 5 \\
\hline Primary (up to 5 grades) & 13.6 & 6 & 11.8 & 12 & 0.0 & 0 \\
\hline Middle (6-8 grades) & 2.3 & 1 & 8.8 & 9 & 10.5 & 2 \\
\hline Metric (10 grades) & 9.1 & 4 & 13.7 & 14 & 26.3 & 5 \\
\hline Above metric (above 10 grades) & 34.1 & 15 & 6.9 & 7 & 36.9 & 7 \\
\hline Total & 100.0 & 44 & 100.0 & $102^{*}$ & 100 & 19 \\
\hline \multicolumn{7}{|l|}{ Location } \\
\hline Rural & 61.4 & 27 & 81.1 & 86 & 63.0 & 12 \\
\hline Urban & 38.6 & 17 & 18.9 & 20 & 37.0 & 7 \\
\hline Total & 100.0 & 44 & 100.0 & 106 & 100.0 & 19 \\
\hline
\end{tabular}

*4 out of 106 women in FGDs did not mention their age and education.

\section{Themes for developing the guidelines}

The following three themes guided the conversation/discussion in the IDIs and FGDs.

Contraceptive use and decision making regarding use if there was desire to space or limit children: availability of family planning services in the community, perceptions regarding family planning use, and (in the case of IDIs with recent abortion seekers)whether the respondent ever used contraception.

Unintended pregnancy and abortion: reasons for opting for abortions; the role of women, husbands and other family members in decision-making and accessing abortion services; financial and mobility issues in accessing such services and quality of care at facilities providing abortion services.

Post-abortion care: knowledge and/or experience of post-abortion complications and subsequent health seeking behavior; roles of women, husbands, other family members and community level 
factors in decision-making and accessing PAC services; financial and mobility issues in accessing such services and quality of care at facilities providing PAC services.

\section{Ethical considerations}

Abortion is a highly sensitive topic in Pakistan where it is widely considered illegal and taboo. The study received approval from the Internal Review Board (IRB) of the Population Council, and ethical considerations against all possible risks for respondents in the present study were taken into account. Interviews were only conducted after study specific consent forms werecompletely read aloud to the interviewees and the interviewees gave arecorded verbal consent. The moderatorsthen signed the form on their behalf. All FGDs and IDIs were recorded with respondents' consent. Digital recorders that allow for playback and editing were used in order to aid in transcribing.

\section{Data management and analysis}

All the recorded files were managed systematically by assigning a unique identification number to each interview so as to ensure the anonymity of the respondent. The research team transcribed all interviews from the local language to Urdu and added their personal observations noted during the interviews.

Data analysis was carried out using the "framework approach". This approach allows comparison across cases by themes. Predetermined analytical categories are developed prior to the research (in the form of a topic guide) and analysis is carried out on those themes ${ }^{43}$ (See details of the frameworkapproach used in the analysis in Annex 11). Senior members of the research team read and re-read transcripts for each group of respondents according to predetermined analytical categories and organized individual responses in matrices developed in Microsoft Excel for analysis. Summary matrices were developed for every category of respondents in each district by comparing, contrasting and synthesizing information within and across cases.

\section{4: Findlings}

Views on unintended pregnancy, family planning, abortion and post-abortion care were included in the interviews. Due to the large body of rich data that was generated we selectively focused on women's barriers in decision making and accessing post-abortion care services in this chapter.

According to respondents in LHW-served areas and service providers, once the decision to terminate the pregnancy has been made, a woman will first try various methods to end the pregnancy at home. Initially, she will typically use herbal remedies or medicines prescribed by a Dai or suggested by a family member or friend. However, the general perception of respondents is that these remedies are ineffective. Having failed to terminate the pregnancy at home the 
woman usually gives up, resigned to continue the pregnancy, or decides to seek 'professional' services.

These views were endorsed by women having recently sought an abortion. A number of women (18 out of 44) first tried home remedies while the others went directly to a private service provider (doctor, LHV, Nurse or a dai) for an abortion.

\begin{abstract}
"Firstly, women try to terminate their pregnancy at home. They drink different herbal infusions. Now the trend has changed, now they know that these things cannot cause abortion, but they start these things at home for termination of pregnancy then come to hospitals. In this process they waste two months after that they came to us for help or ask about any service provider and expenses of abortion" (IDI-LHW, Rawalpindi).
\end{abstract}

"These herbal teas etc. do not work and there is little chance of abortion with them"(FGD, Rawalpindi).

"I went to a NGO clinic. She cleaned me with an equipment and also used a machine. The procedure took half an hour"(IDI-women, 40 years, no schooling, 10 children, Umerkot).

\title{
Post-abortion complications and barriers to seeking care
}

Out of the 44 women who had had an induced abortion in the 6 months preceding the study, only 11 did not develop any complications, while the remainder (33 women) complained of a range of problems.

It is apparent from the IDIs that most women experiencing post-abortion complications rely on home remedies either as the first step or as their only attempt at treating their complications. IDIs with service providers suggest the same. The most frequently used home 'remedies' as reported by these women was a healthy and nutritious diet which includes having milk, fruit, and juices. Other home remedies mentioned were mint infusions, cinnamon infusions, green tea, black tea, eggs, spinach and phaki, a mixture of ground herbs. Service providers added ground tamarind, pulses and nuts, crushed betel nut, cumin seeds, clarified butter (ghee), and mixed melon seeds (chaarmaghz) in ghee, to the list of home remedies. Some women self-medicated with calcium and iron tablets, multivitamins and pain killers such as Ponstan and Paracetamol.

\footnotetext{
"When I had bleeding for 15 days my in-laws told me to boil some mint, ajwain(black henbane seed) and cinnamon and drink the infusion. I did so, but my bleeding did not stop although the flow did become lighter and a large piece came out from uterus,
} 
something like a child"(IDI-woman, 30 years, 10 Years of schooling, 4 children, Karachi).

The most commonly experienced problems were general weakness followed by bleeding and infection. About one third of the women who experienced complications (12 out of 33) said that they either had heavy bleeding, prolonged bleeding or irregular bleeding following the abortion procedure and one fourth experienced fever, headache and infection along with other complications.

When women and service providers described the possible immediate and long-term complications following an induced abortion,they almost always mentioned pain in the lower abdomen and back. However, according to them heavy bleeding was by far the most common post-abortion complaint.

"Major complications are bleeding and swelling, pain in the lower abdomen; spotting and minor problems are nausea, giddiness, loss of appetite, and anxiety. Sometimes they may have fever after abortion which may be low or high grade.Sometimes women come with $100^{\circ}-103^{\circ} \mathrm{F}$ fever three days after having had an abortion" (IDILHV, Karachi).

"After a D\&C, I started having heavy bleeding and fever. I was feeling severe weakness and backache. I also had to take care of my children and that is why I faced many problems and I could not recover for a long time"(IDI-woman, 32 years, 14 years of schooling, 3 children, Rawalpindi).

\section{"Immediately after the abortion I had severe pain in my lower back and chest. There was weakness and I thought that I was going to die. I had continuous fever for 15 days after my abortion"(IDI-woman, 31 years, 4 years of schooling, 7 children, Hafizabad).}

When women do experiencepost-abortion complications, they need to have treatment, but it is not easy for a woman to go directly to seek treatment.Instead, women have to go through many steps before reaching a decision of whether to seek PAC or not. In the process of decision making there may be a number of players who have roles, including the woman herself, her husband, her mother-in-law, and other family and community members, which can cause delays in seeking services.

During IDIs with women who had an abortion and service providers in their vicinity, it was found that across all communities (both urban and rural), the decision to seek PAC services is not the woman's to make alone. Inalmost all cases women have to consult their husbands and seek their approval before going to a service provider. 


\begin{abstract}
"I decided by myself to seek treatment and after taking permission from my husband I went with the LHW to the LHV to get medicine"(IDI-woman, 36 years, 12 grades, 3 children, Rawalpindi).
\end{abstract}

"She has to take her husband's permission for PAC as she needed his consent at the time of abortion. A woman cannot go alone without husband's permission"(IDI-TBA, Hafizabad).

The general opinion of women and service providers regarding decision-making for PAC was that without their husband's permission, it is hard for women to access PAC services both in terms of finances as well as mobility/logistics. Women's ability to decide on seeking PAC independently, without consulting their husband, depends to some extent on their personal financial situation.Women who do not need their husbands to pay for the services can decide independently whether or not to seek PAC. However, as most women in Pakistan are economically dependent on their husbands, the decision is usually a joint one.

\footnotetext{
"Women who are self-sufficient and economically empowered, who do not have any hurdles in their way, can (seek treatment) themselves. But women like us who are dependent on our husbands have to wait for them to come and give us money and go along with us to the health facility" (FGD Hafizabad).
}

Another situation in which a woman can make this decision on her own is if she is facing severe complications in the absence of her husband or if services are available free of cost.

\footnotetext{
"If a woman has serious complications she can go for treatment without asking her husband and does not necessarily need any permission"(IDI-woman, 40 years, uneducated, 6 children, Umerkot).
}

"If there is a facility for free treatment, the woman will not even think about seeking permission, she will only have to inform her husband. If the treatment is free then the woman will not have any decision making issues, even the mother-in-law will not be a problem and there will be no barrier in her way"(FGD, Karachi).

\title{
Support of husbands
}

Husbands generally play an important and supportive role in accessing PAC services. Indepth interviews reveal that husbands are concerned when their wives develop postabortion complications:

"My husband supported me. He was observing the problems I was facing in managing the household and my health was suffering. Then he asked me to see the doctor. I went to doctor on his advice. He brought me fruits and juices, he would say 'your 
health is essential so that you can take care of the children"(IDI-woman, 32 years, 14 grades, 3 children, Rawalpindi).

"My husband is very supportive; he forcefully took me to hospital (to get treatment for complications). He said if you die I have nothing here. He's a good man" (IDI-woman, 30 years, 10 grades, 4children, Karachi).

The support primarily translates as arranging money for transport and treatment, fetching medicines in cases of mild complications and, in cases of serious complications, accompanying their wives to a health facility or service provider. Arranging money often involves borrowing the required amount or selling a household item to bear the costs of treatment.

"He knew that I was suffering (from post-abortion complications) and we should go to
the same doctor. He took care of my health through a healthy diet and also
accompanied me for the follow up"(IDI-woman, 12 grades, 36 years, 4 children,
Rawalpindi). "If a situation arises where we have to go for medical services then even if he will have to sell something for the money he (husband) will do so"(IDI-woman, 28 years, uneducated, 4 children, Khanewal).

While husbands were generally supportive a few women mentioned that due to financial constraints they could not support them in seeking PAC services, even if they wanted to.

\begin{abstract}
"My husband asked me to see a doctor so I asked him to give me money. He said our economic situation is in front of you: whatever I earn I give to you. What he gives me is spent on entirely on the children's expenses and rent for the house, so I had to bear the complications without treatment"(IDI-woman, 30 years, 10 grades, 5 children, Karachi).
\end{abstract}

"I told my husband about the complications and he said I don't have money what
should I do" (IDI-woman, 18 years, uneducated, 1 child, Umerkot).

Furthermore, men's knowledge of medical complications following abortion was very limited, as gauged from the informal discussions(men from onlyfourcommunities mentioned these complications). Since husbands are the main decision makers for accessing care, this lack of knowledge may create a hindrance in accessing care for women as they will not be able to realize the full importance of the severity of the complication. 


\section{The role of mothers-in-law and other family members}

When asked about their perception of the role of mothers-in-law and other family or community members in the decision-making process regarding the treatment of post-abortion complications, women and service providers across the board said that they had little role in this process. Changing patterns of family type from joint to nuclear are a contributing factor in the waning role of mothers-in-law.However, in some cases where the mother-in-law was part of the household unit, her role in the decision to seek PAC services was reported to be negligible and at times supportive.

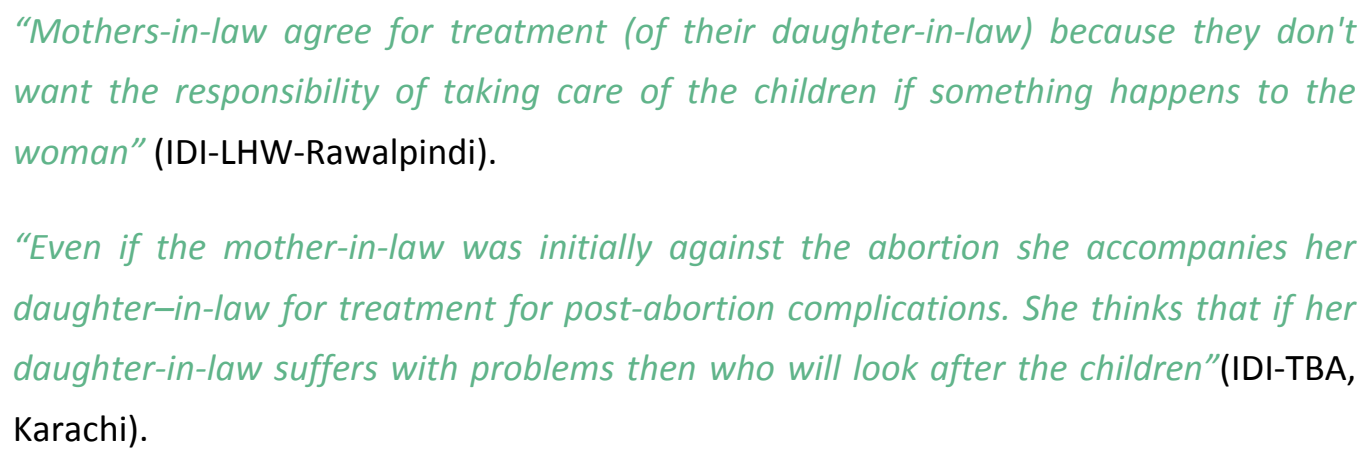

Other family members have no role at all except when a woman is suffering from severe complications.

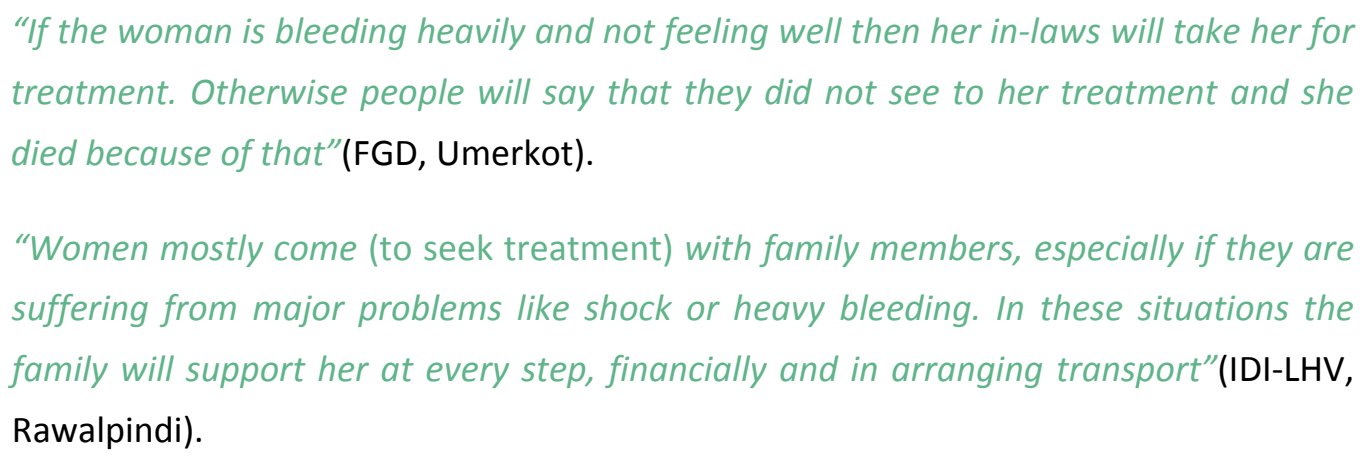

In the more remote communities, not served by LHWs, which also tend to be more conservative, the mother-in-law could have a strong negativeinfluence. FGD participants in these communities gave the impression that, if a woman develops post-abortion complications, her mother-in-law will not support her treatment, because in these areas induced abortions are considered to be a sin and there is a preference for large families. Moreover, in these more conservative areas the joint family system was pervasive and hence the role of the mother-in-law was significant in deciding whether or not to seek post-abortion care.

\footnotetext{
"When a woman faces complications then her mother-in-law says that you have limited my grandsons. So now you will have to bear these problems"(FGD, Sanghar).
} 
"When a woman faces complications after an abortion, women of the community say

that she has committed a sin and that is why she is facing these problems"

(FGD, DG Khan).

\title{
Issues of Mobility
}

Women may also face another important hurdle because of their limited mobility. This encompasses different aspects, such as their ability to travel alone, the distance to health facilities and the need to arrange transportation. Respondents mainly referred to geographic mobility and distances to travel as a barrier in accessing PAC services.

Mobility issues are of lesser concern in urban areas because services are readily available within these communities. Service providers interviewed in the urban community in Karachi also perceived no such issues, as facilities are available within their community.

\begin{abstract}
"Women living in this community can easily access the facility and they need no transport. People have good social relationships therefore women do not face mobility issues as they are accompanied by their friends or neighbours."(IDI- LHV, Karachi).
\end{abstract}

In rural areas women's mobility does come in to play. At least a few women from all three rural communities reported that they could not go out alone and required a chaperone, either a male relative (husband or a son) or a community health worker, to accompany them to seek treatment.

\footnotetext{
"You may take a child along with you even from neighbourhood but you cannot go alone. If I go alone people gossip why is she going alone and ask where her husband is. Even if we have a five year old kid we take him along but we do not go alone" (FGD, Umerkot).
}

In rural areas, transportation is also a hindrance to mobility. This was confirmed by service providers: in Hafizabad, Khanewal and Umerkot, three of the more rural communities, providers said that women in their districts have to face problems in accessing PAC services.

\section{"Women have to walk 2-3 miles to reach us. There are buses in KoloTarar but not in small villages" (IDI-LHV, Hafizabad).}

Mobility issues are linked strongly with the time taken to travel away from home and the potential conflict with domestic responsibilities. It is hard for women to free themselves from household chores and leave the children unattended at home. 


\begin{abstract}
"Leaving the house is a big problem for women. At times she even has to leave her children home alone, locking them inside the house as there is no one to stay with them"(IDI-LHV, Hafizabad).
\end{abstract}

\footnotetext{
"Women have household problems as nobody is at home to look after her children. She manages to visit the clinic once but the next time she does not have anybody to help her out at home so she never visits (for follow-ups)" (IDI-LHW, Rawalpindi).
}

\title{
Arranging the Finances
}

If a woman develops complications from an unsafe abortion, many factors may hinder her from accessing the treatment she needs at a health facility. The possible barriers include costs, availability of nearby services and family commitments.

In both urban and rural communities, financial constraints stood above all other barriers and problems in accessing PAC services. Women in the more developed district of Rawalpindi in Punjab were the only ones who did not cite financial constraints as such.This was because the families were relatively better off so they did not face any financial problems in accessing PAC services and have better access to health services in their area.

\section{"I had to face financial problems to some extent however it was not very hard to manage. My family members were very cooperative, that is why I did not face any real barriers" (IDI-woman, 32 years, 14 grades, 3 children, Rawalpindi).}

But apart from a couple of cases, all women (both urban and rural) had to face serious financial constraints in accessing PAC services.Money is required not only for treatment but also for transportation to the health facility. Considering these issues, sometimes women prefer to bear the complications and not seek treatment at all.

\footnotetext{
"I have no money. I am already worried about returning my loan (she borrowed money for her abortion). I have become weak because I don't get enough to eat. If I visit the doctor again I will have to pay a fee for the consultation and then I won't be able to buy the medicines she prescribes. If I had money I'd eat properly and maybe not require medicines. I am bearing pain and all problems due to shortage of money" (IDI-woman, 30 years, 10 grades, 5 children, Karachi).

"I developed an infection in my abdomen (uterus) after induced abortion. I tried to manage my complication at home by just having black tea (kehwa) and boiled egg because I could not afford to have milk, soups or meat etc. Then I sold my fan and went to a doctor for treatment" (IDI-woman, 32 years, 4 grades, 9 children, Hafizabad).
} 
Sometimes, the priorities are different and children's future trumpsthe woman's health, as in the case of this poor respondent:

"My husband wants me to seek PAC treatment but I myself don't go to the doctor. I want that the money to be spent on me should instead be spent on my children's education"(IDI-woman, 28 years, uneducated, 3 children, Khanewal).

Those women who did choose to seek treatment managed the finances either through borrowing money or selling crops or a household item. However, sometimes the money collected is enough only for a first consultation and follow-up visits have to be forgone.

"Often women do not go for treatment due to many other expenses involved. Apart from the doctor's consultation fee of 500-1000 rupees, money is required for transportation, and then the doctor will prescribe medicines or ask her to go for clinical tests. Money is the main problem. At the time of abortion women are desperate and they do manage(to collect the money at any cost) somehow. For complications they must first take medicines for 4-5 days and then go for follow-up which again requires money. All this becomes difficult to afford"(FGD, Rawalpindi).

"Yes, we have financial problems- that is why I have not been to the doctor till now for these problems. But now there is hope for some money so I will go. We are poor people we don't have much land which we can sell off for money"(IDI-woman, 40 years, uneducated, 10 children, Khanewal).

\section{Choice and quality of post- abortion care providers}

One of the real issues is whether women themselves take their symptoms seriously and whether their health is given value by their family. Usually women seek care only when complications have become more serious. Women usually ignore their mild or moderate symptoms and avoid seeking care due to financial pressure and household responsibilities. There is a sharp contrast in the desperation seen in women at the time of seeking abortion that is not seen for seeking care for post-abortion complications. At the time of abortion they consider health as one of the main reasons for having an abortion but this is not seen as a priority at the time of complications.

Service providers also said that when women ignore or delay seeking treatment, the complications become more serious and women end up spending a lot more money on their treatment.

\footnotetext{
"They have no idea that they have infection and this infection can increase. They do not use medicines due to financial problems. Medicines are expensive and they reach
} 
at last stage of complications when they rush to hospital or to the doctor"(IDI-LHV, Rawalpindi).

A number of women(6 out of the 33) who reportedly developed either mild or serious complications as a result of induced abortion did nothing to treat their problems, mostly due to their dire financial circumstances.

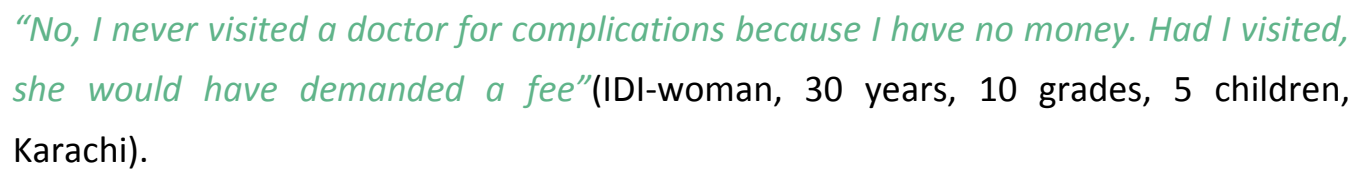

Another 14 out of 33 women, who developed complications, took home remedies for their complications and almost half of these ultimately ended up going to a service provider for treatment. Thirteen women out of the 33 who developed complications reported that they went directly to a service provider for the management of their complications.

When asked about the availability of PAC services in their areas, respondents in all categories (in well-served communities - both urban and rural) identified various providers and facilities in the urban areas (Karachi and Rawalpindi), both public and private. However, in rural communities (Khanewal, Umerkot and Hafizabad), women had to travel quite some distance before they could get appropriate care. The common providers available were LHVs, nurses, community workers and Dais. A few clinics run privately by doctors and social marketing group Greenstar also provide PAC services. Needless to say, in the under-served areas studied, there were no such services available at a convenient distance. So access to services is a real barrier to being able to get postabortion complication care, as treatment is only available in towns and cities.

"No, there is no facility in our area we go to Hafizabad city" (FGD, Hafizabad).

"There are no facilities over here. This is a big village but still there are no facilities, we have to go to Umerkot city"(FGD, Umerkot).

\section{Level of care from service providers}

Service providers reported that women usually seek treatment of their post-abortion complications in the private sector with only a few poor women going to government hospitals. According to service providers, the reasons for women frequenting the private sector providers rather than public sector providers are a) the rude behavior of doctors and other staff at public 
facilities; b) lack of proper facilities/medicines in the public hospitals; c) the availability of private facilities in the community and the inconvenience of often distantly located government hospitals.

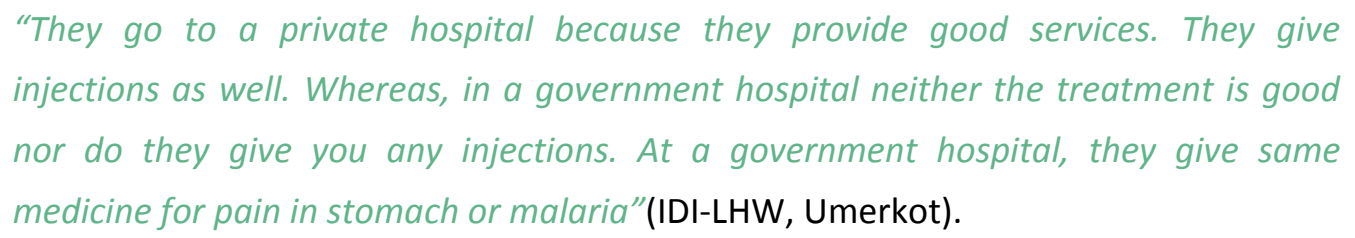

An overwhelming majority of the women who had post-abortion complications confirmed these views: most said that they sought treatment for their complications in the private sector, preferring to return to the same service provider who initially induced their abortion. Most women consulted a female doctor at these facilities. Very few went to a female paramedic (LHV/nurse), only one mentioned that she sought treatment from a Dai (TBA), and one other consulted a community health worker.

\footnotetext{
"I was feeling weak (after having abortion) then I went to the same doctor (private) who provided me with abortion services. You see, if I had gone to any other provider, I would need to tell them my whole situation again. It was convenient and preferable for me to go to the same doctor where I did not need to restart anything"(IDIWoman-36 years, Intermediate, 4 children, Rawalpindi).
}

In discussions with men it was learnt that the treatment seeking pattern for post-abortion complications depends on the availability and quality of services and their affordability, as wellasthe awareness of the patient in regard to these factors. Men reported that at the initial stage, women facing complications mostly consult community level service providers (LHVs and dais). At the second stage,women consult with qualified service providers either at public or private facilities on the basis of affordability. Poor women are more likely to seek treatment at public health facilities while those who can afford it, go to private sector providers.

\begin{abstract}
"Women who go to private facilities have money while those who are poor and cannot afford it, go to a government hospital. In a government hospital neither is there a doctor nor is the treatment good. We mostly go to government facilities because we are poor and do not have a lot of money and the service provider at the private hospital obviously charges money"(FGD, Umerkot).
\end{abstract}

\title{
Methods used by service providers for PAC
}

The method of treatment depends on several factors: severity of the complications, skills of the service providers and the socio-economic condition of the woman. Methods most frequently 
mentioned by service providers for treating complications are injections and tablets, intravenous (IV) drip, antibiotics, D\&C and blood transfusions.

\begin{abstract}
"If a woman has heavy bleeding then she will go to government hospital where service providers may perform a D\&C. In some cases, women may need a blood transfusion. If she is going into shock, then they will treat her accordingly. They will give her antibiotics and proper treatment. If she has an infection or sepsis, she may die, if not properly managed" (IDI-LHV, Rawalpindi).

"After two days of abortion, heavy bleeding started. My husband was worried about my condition; we went to the same clinic (where I had abortion). They asked us to arrange two bottles of blood (my brother in law donated one bottle), my husband arranged another. They treated me and then I was alright. This was totally my fault that after abortion when I came back home, I did not rest and started doing all household chores" (IDI-30 years, 10 years of schooling, 6 children, Karachi).
\end{abstract}

The use of Misoprostol (mentioned as tablets) for the purpose of treating incomplete abortions was mentioned by providers and women themselves.

\footnotetext{
"When women have complications, they are usually treated by providing medicines, injections and tablets" (IDI-LHV, Khanewal).

"I went back to the LHV, 8 days after abortion when my bleeding did not stop. She gave me two very small tablets to eat and advised me to eat fruits as much as I could. My bleeding stopped after I ate those tablets but again it started, then I repeated the dose and now I am alright"(IDI-27 years, 10 years of schooling, 3 children, Khanewal).
}

\title{
Behavior of the service providers
}

When asked about the behavior of service providers consulted for PAC, women who had had an abortion gave a mixed response, although a substantial number were satisfied with the behavior of service providers.Many went to the same provider who had performed their abortion.However, some women were unhappy with providers they visited.

"Yes they are considered experts in their field. It is a big hospital run by both husband and wife. People praise them but for me they are not experts because my (health) problem could not be fixed by them" (IDI-Woman-30 years, 10 classes, 03 children, Karachi). 
FGDs with women and informal discussions with men revealed that the behavior of private providers is considered to be better than of providers in the public sector. For that reason and others they prefer to go to the private sector with their complications.

\footnotetext{
"Those who are paid, show good behavior. In a public hospital we do not pay, therefore they don't behave properly with us; they don't even examine us properly" (FGD, Umerkot).
"Those who have money go to a private hospital as the treatment is good over there and those who are poor go to government hospitals"(FGD, Umerkot).

"Public sector providers behave rudely with women and don't give proper time to them. Dais are good but they are not skilled" (FGD, Khanewal).
}

Similarly women in under-served areas, despite their poor socio-economic status and the higher expenses of the private sector, favoured private providers over public because of differences in the quality of services and thebehavior of service providers.

\footnotetext{
"It is our compulsion to go to private facilities because in public hospital treatment what is done in 10 days but the same treatment is done within five days in private facilities. Though we have to spend money but the private service is better than public because we cannot leave our children and animals behind for so many days" (FGD, Sanghar).
"At a private facility all type of treatments are available at one place whereas at public facility, one has to run here for one thing and elsewhere for another. This does not happen at private facility" (FGD, Sanghar).

\section{Family Planning counselling}

Post-abortion family planning counselling and provision of contraceptives is a crucial part of PAC. However,IDIs and FGDs with women showthat very few providers counsel on family planning even when treating a woman with post-abortion complications.

"I asked for her advice and then she only suggested. I use condoms to avoid
unwanted pregnancy" (IDI-Woman, 33 years, 10 years of schooling, 4 children, Rawalpindi).

"She (the service provider consulted for PAC) did not provide any contraceptive but because I knew about it I got an IUCD inserted from another doctor later on" (IDIwoman, 35 years, no schooling, 6 children,Umerkot). 
Only $\mathbf{3}$ of the $\mathbf{3 3}$ women who sought PAC were actually provided with a contraceptive methodas part of their treatment. Two women each from Karachi and Rawalpindi were given pills while one woman from Khanewal was provided with an injectable.

"The doctor gave me pills (at the time of PAC), I used those pills for some time but discontinued due to the side effects"(IDI-Woman, 30 years, 10 years of schooling, 3 children, Karachi).

\section{5: Conclusions}

The study suggests that women who experience post-abortion complications face certain barriers both at the time of decision making and when accessing PAC services. At the decision making phase, husbands take the lead andthe woman's own role is limited except in the few situations where she is financially independent, services are nearby or provided free of cost. Mothers-in-law and other family and community members have very negligible roles in decision making. Cost and mobility issues emerge as the main barriers in accessing PAC services, particularly for those who are rural and poor.

These hurdles contribute to delays in seeking PAC services because women's determination towards seeking PAC service is quite weak as compared to when they seek services at the time of abortion. Women start their treatment through home remedies and do not go to service providers unless complications become serious.

The women who seek treatment prefer to return to the (private sector) service provider who carried out the abortion in the first place. There are two main reasons for this: the service provider already knows the patient's history, and the quality of care, particularly the behavior of the provider, is better than what is available at public facilities. However, reaching health facilities does not mean that the women will receive treatment with high quality of care; besides other gaps, family planning counselling is one of the important elements of PAC not provided by most service providers. 



\section{Chapter 8 \\ Summary of Key Findings and their Implications for Policies and Programs}

This study provides the type of information on the condition of abortion practice, post-abortion complications and post-abortion care that should be of use and interest to a number of key stakeholders: public health professionals and practitioners, gynaecologists and obstetricians, policy makers, researchers, activists and academicians both within Pakistan and internationally.

\section{1: Conclusions}

- The type of method women in Pakistan are using to end an unwanted pregnancy has changed in one important way since 2002: the use of misoprostol (medication abortion) among urban women has increased dramatically, from being non-existent in 2002 to being considered the leading method by one-third of knowledgeable health professionals in 2012.

- While D\&C was believed by $75 \%$ of health professionals to be the leading method among all women obtaining abortions in 2002 , by 2012 , only $25 \%$ thought it was the leading method in urban areas, and only $24 \%$ in rural areas. Among women inducing their own abortions, the perceived rise in misoprostol use was from $9 \%$ in 2002 to $44 \%$ by 2012 .

- At the same time, there appears to have been little change over the decade in the type of provider women go to for an abortion.

- The average cost of a first-trimester abortion is estimated to have risen substantially over the past decade. After accounting for inflation based on Federal Bureau of statistics data on inflation, the cost have hardly changed across the two time periods(2002 and 2012). The estimated cost has specially increased for abortion carried out by the type of provider most commonly used by poor women-LHVs/Nurses/Midwives.

- $\quad$ For both poor and wealthier, and for both urban and rural women obtaining abortions in 2012 , the greatest risk to their health is from procedures carried out by TBAs and Dais. The risk of abortion-related complications has remained virtually unchanged for procedures carried out by every type of provider except pharmacists/drugstores, where it has risen, and lay practitioners, where it has fallen. 
- Among women who do experience abortion-related complications, there was a slight increase between 2002 and 2012 in the proportion believed to obtain the post-abortion treatment they needed. In 2012, this proportion was lowest among the rural poor-57\%- and highest among non-poor urban women-86\%.

- Once women with abortion-related complications have reached a health facility, the techniques they are most likely to be treated with are D\&C and D\&E, despite the fact that the doctors in those facilities believe that the preferred technique by far would be misoprostol.

- Most health facilities offering PAC are equipped with functioning D\&C sets, but very few facilities below the level of teaching hospital are equipped with functioning MVA or EVA kits.

- Doctors working in both the private and the public health sector facilities that provide postabortion care (PAC) believe that staffing levels are largely inadequate. The absence of gynaecologists and anaesthetists in many health facilities in the public sector is the most striking deficit.

- In 2012, an estimated 692,000 women with abortion-related complications were treated in health facilities. Six out of 10 PAC clients were treated as out-patients and the same proportion were treated in private-sector facilities.

- The number of PAC patients treated in health facilities in the public sector in Pakistan rose only slightly between 2002 and 2012 (from about 246,000 to about 269,000), and population growth accounted for much of this increase.

- In 2012, 15 women were treated for post-abortion complications for every 1,000 women aged 15-49, of which an estimated 6 per 1,000 reflects women treated in public-sector facilities, and an estimated 9 per 1,000, those treated in private-sector facilities.

- Even though a majority of health facilities offering PAC services report giving clients family planning counselling and contraceptive services, many health professionals interviewed in 2012 considered the counselling on a range of topics to be inadequate.

- Four out of 10 doctors in the Health Facilities Survey thought that providers have negative attitudes toward PAC patients, and three out of 10 thought providers were reluctant to treat these women, 
- Approximately four out of 10 health professionals and doctors working in health facilities that offer PAC consider that abortions sought by women who have been raped are not permissible.

\section{2: Discussion}

The treatment of women suffering post-abortion complications imposes a heavy burden on the health system in Pakistan.Every year almost 700,000 women present themselves with postabortion complications in Pakistan which involves a huge economic cost to manage the complications. This puts a significant financial burden on the already fragile and compromised health care systems of developing countries, such as Pakistan and therefore results in reduction of their capacity to provide other much needed services ${ }^{44}$. Despite this heavy caseload, many more women do not reach the kind of health facility surveyed in this study. Not knowing that such facilities exist, how to find them, how to get to them and the inability to pay for travel or take time off from work are probably some of the major reasons that women with abortion-related complications do not receive the care they need.

Poor rural women are both more likely to go to unsafe abortion providers or use unsafe abortion methods and least likely to obtain care for post- abortion complications. One explanation for this pattern is that the cost of an abortion has nearly doubled over the last 10 years. The increase has been relatively greater for poor women (both rural and urban) because the fees charged by the service providers they most commonly go to (LHV/Nurses and TBA/Dai) appear to have risen the most. Another qualitative study conducted in 2010 in Pakistan found that financial constraints compel women to resort to the informal low-cost services ${ }^{45}$.

Although a higher proportion of poor women in Pakistan are likely to seek PAC today than 10 years ago, only half of poor women who develop post-abortion complications obtain treatment, probably because they cannot afford to do so.

The private sector plays a prominent and dominant role in the provision of PAC services in Pakistan today: twice as many cases are treated at private facilities as in the public health sector. One study claims that the private sector caters to $70 \%$ or more of all health care needs in Pakistan $^{46}$, so it is hardly surprising that the treatment of post-abortion complications is tilted towards the private sector. Nevertheless, public-sector facilities in all provinces have a higher average annual caseload than do private-sector facilities.

The average caseload at teaching hospitals in the public sector rose between 2002 and 2012, which is not surprising given that these facilities are located in mega-cities and accept patients coming from a vast area of the region/province, and that they are the destination for the referral of the more serious types of abortion-related complication. Caseloads in public-sector teaching 
hospitals are probably also rising as patients from other districts or remote areas bypass their own DHQs and THQs to come directly to these hospitals. Or perhaps the number of women experiencing post-abortion complications in the larger urban areas has risen. In contrast, a decline in the size of the annual caseload served by DHQs and to some extent by THQs, could possibly be the result of a shift towards the use of private hospitals in by women living in urban and semiurban areas.

It is possible that the prevalence of severe post-abortion complications in Pakistan may have declined. Evidence for this hypothesis rests in the findings regarding misoprostol, which is growing more popular in Pakistan as a way for women to end their unwanted pregnancies. This method, if used correctly, has few or no complications. And even, if used incorrectly, a normal consequence would be an incomplete abortion without serious physical damage to the women. Previous research suggests that misoprostol is a safe, inexpensive method for inducing abortion, and leads to fewer complications and consequently shorter hospital stays ${ }^{47}$. The dramatic increase between 2002 and 2012 in the use of misoprostol to induce abortion and the concomitant decline in the use of less safe and less effective methods such as laminaria sticks, IUCDs and anti-malarial medication could reflect an improved awareness on the part of women that these earlier traditional methods can have adverse health consequences. It could also reflect the fact that the availability of and access to alternative, newer abortion methods and alternative abortion services has expanded in Pakistan.

While most health facilities offering PAC services have the ability to provide post-abortion care of some kind, there is still room for improvement. For instance, there has been no change over time in the use of D\&C to treat post-abortion complications. Yet when asked about the best procedure for treating complications from a first-trimester abortion, the majority of respondents (in both the public and private sector) recommend misoprostol. Thus, there is a huge gap between the actual and the ideal PAC practice in Pakistan. (The fact that so few doctors in facilities providing PAC services cited MVA and EVA as the best techniques to use on patients with abortion-related complications suggests that a large number of the trained practitioners working in these facilities are not up-to-date with the safest techniques). World Health Organization recommends safer methods for PAC and considers that use of D\&C results in more severe and frequent abortion-related complications ${ }^{48,49,50}$.

Worldwide research shows that Use of contraceptives for post-abortion contraception was associated with decreased risk of repeat abortion ${ }^{51,52}$. Current study shows that while many facilities provide family planning services on their premises there are some limitations in what contraceptive methods are offered (pills, injectables and IUDs, for example, are much more likely to be available than implants or sterilization). More importantly, in most facility types, only 
around half of clients leave with a method. A large number of facilities refer clients to other facilities to obtain contraceptives; however, there is lack of coordination and referral linkages between the public and private sectors, and between the health and population departments. But although counselling services are being provided in the facilities, the quality and extent of these is inadequate. Thus, one of the greatest opportunities for preventing unwanted pregnancies in Pakistan and, by association, induced abortions, is being missed: the provision of a contraceptive method to a much higher proportion of women leaving a health facility after receiving PAC treatment.

The finding that many providers in health facilities offering PAC services have negative attitude towards the patients and are reluctant to treat them could have serious consequences for women in the community experiencing abortion-related complications. These discriminatory attitudes are surely well known outside the hospitals and must certainly discourage many women from seeking the care they need.

\section{3: Recommendations}

- To reduce the number of women in Pakistan in need of post-abortion care, access to quality family planning services that will help women avoid unwanted pregnancies must be made more widely available, especially in rural areas. An expansion of planning services will require the training of health providers and the uninterrupted availability of contraceptive supplies.

- Health care providers, including mid-level providers, working in the Health Departments and the Population Welfare Departments should all receive training in non-invasive PAC procedures such as manual vacuum aspiration (MVA) and misoprostol. MVA training should be made a part of all medical undergraduate training curricula. All existing health providers in facilities offering PAC services should be briefed on the use of misoprostol as an effective means of treating incomplete abortion.

- It is also very important to train mid-level providers (LHVs, FMTs and nurses) in family planning, especially in the primary health care facilities (RHCs \& BHUs) where most rural and poor women initially go for treatment. These are the facilities where, if proper counselling and family planning services were available, women with unmet need would have easier access to quality services that could help them avoid unwanted pregnancies.

- There is a generalized bias of health care health professionals against mid-level providers, they are seen by them as not been competent for providing PAC services. A national 
consensus on providing training to mid-level providers could be developed by arranging national and provincial seminars. For this purpose, the newly established Ministry of National Health Services, Regulations \& Coordination should be brought on board to issue necessary policy directives.

- Misoprostol has now been included in the Essential Medicines List (EML) in all the four major provinces, which should ensure its availability in government health care facilities. Nevertheless, continued advocacy for its implementation is required.

- PAC protocols that conform to the latest scientific advancements need to be developed. These should be widely disseminated along with service guidelines for PAC.

- There should be closer coordination between the Health Department and the Population Welfare Departments to develop an inter-departmental referral system and more efficient referrals between facilities in the public and private sectors and for ensuring regular supply of contraceptives.

- There is a strong need to ensure that quality counselling and contraceptive services are provided to both spontaneous and induced abortion clients within health facilities at all levels of the public and private sectors. This should be an integral part of the regular services being provided. There is also a great need to train providers in proper techniques of counselling to make them more client-centred and to develop their skills in IUCD insertion and removal techniques (including postpartum IUCD insertion), proper infection prevention practices and the management of side effects to provide better and safe services for post-abortion care patients.

- Address widespread negative attitudes about women who need treatment as a result of unsafe abortion and try to lessen the discrimination toward this group expressed by many providers. 


\section{References}

1. Sathar ZA, Kazi S. Women's autonomy, livelihood and fertility: A study of rural Punjab. Pakistan Institute of Development Economics. Islamabad. 1997.

2. Arif S, Kamran I. Exploring the choices of contraception and abortion among married couples in Tret, rural Punjab, Pakistan. Islamabad. Population Council. 2007.

3. Gazdar H, Khan A, Qureshi S. Measuring the economic costs of unsafe abortion mortality and morbidity in Pakistan: Preliminary findings and survey design. Karachi. Collective for Social Science Research.2010, January.

4 National Institute of Population Studies (NIPS) and Macro International Inc. Pakistan Demographic and Health Survey 2006-07. Islamabad. NIPS and Macro International Inc. Islamabad (Pakistan). 2008.

5 Casterline J, Arif S. Dealing with unwanted pregnancies: Insights from interviews with women. Islamabad. Population Council. 2003.

6 Casterline J, Singh S, Sathar ZA. Unwanted pregnancy and post-abortion complications in Pakistan: Findings from a national study. Islamabad. Population Council.2004, October.

7 Sathar ZA, Singh S and Fikree FF. Estimating the incidence of abortion in Pakistan.Studies in Family Planning. 2007. 38(1): 11-22.

8 Rehan N, Inayatullah A, Chaudhary I. Characteristics of Pakistani women seeking abortion and a profile of abortion clinics. Journal of Women's Health \& Gender-based Medicine. October 2001. 10(8): 805-810.

9 Korejo R, Noorani KJ, Bhutta S. Sociocultural determinants of induced abortion. Journal of College of Physicians and Surgeons, Pakistan.2003, May. 13(5): 260-262.

10 Gilani, S, Azeem P. Induced abortion: A clandestine affair. Journal of Postgraduate Medical Institute, Pakistan. December 2005. 19 (4): 412-5.

11 The Alan Guttmacher Institute. Adding it up: The benefits of investing in sexual and reproductive health care. New York. The Alan Guttmacher Institute. 2003.

12 Abortion in Pakistan: Morality becomes more restrictive than the law. The Asia Safe Abortion Partnership Blog. January 4, 2013 [Homepage on Internet; cited 2013 June 17]. Available from: http://asap-asia.org/blog/abortion-in-pakistan-morality-becomes-more-restrictive-than-the-law/

13 Kariapper R. Review of abortion material in Pakistan. ShirkatGah. [Homepage on Internet; cited 2013, June 17]. Available from: http://www.shirkatgah.org/_uploads/_files/f_14abortion_material_in_pak.pdf

14 Ahsan A, Jafarey SN. Unsafe abortion: Global picture and situation in Pakistan. Journal of the Pakistan Medical Association. 2008. 58(12): 122-127. 
Jaffrey SN.Maternal mortality in Pakistan-compilation of available data.Journal of the Pakistan Medical Association. 2002. 52(12): 539-544.

Mahmud G, Mushtaq Z. The Incidence and outcome of induced abortions at one of the hospitals of Islamabad, Pakistan. Population Association of Pakistan. 2001.

Pakistan Bureau of Statistics. Household Integrated Economic Survey (HIES), 2011-12. Government of Pakistan.Statistics Division. Islamabad. 2013, May. International Conference on Population and Development (ICPD). International Conference on Population and Development Programme of Action (ICPD). Cairo, Egypt. 1994, September 5-13.

Solo J. Easing the Pain: Pain management in the treatment of incomplete abortion. Reproductive Health Matters. 2000. 8(15): 45-51.

Government of Pakistan. Karachi Declaration on scaling up MNCH-FP Best Practices in Pakistan. Ministry of Health and Ministry of Population welfare. 2009, October 1-2.

Rashida G, Shah ZH, Fikree F, Faizunnisa A, Mueenuddin LI. Abortion and post-abortion complications in Pakistan: Report from health care professionals and health facilities. Islamabad. Population Council. 2002.

22 Prada E, Kestler E, Sten C, Dauphinee L, Ramírez L. Abortion and post-abortion care in Guatemala: A report from health care professionals and health facilities. Occasional Report No. 18. New York. Guttmacher Institute. 2005.

23 Paulin P, Moore AM, Singh S, Remez L, Birungi F, Nyirazinyoye L. Unintended pregnancy and induced abortion in Rwanda: Causes and consequences. New York. Guttmacher Institute. 2012.

24 Prada E, Mirembe F, Ahmed FH, Nalwadda R, Kiggundu C. Abortion and post-abortion care in Uganda: A report from health care professionals and health facilities. Occasional Report No. 17. New York. The Alan Guttmacher Institute. 2005.

Population Council. Mapping of health and reproductive health services-Survey of service delivery points. Islamabad. Population Council. 2010.

Government of Pakistan. Pakistan Economic Survey. Ministry of Finance [homepage on the Internet; cited 2013 June 13]. Available from: http://www.finance.gov.pk/survey/chapter_12/07-Inflation.pdf

27 Mayo Clinic [homepage on the internet]. Medical abortion. 2012 [cited 2013 Feb 28]. Available from: http://www.mayoclinic.com/health/medical-abortion/MY00819/DSECTION=what-you-can-expect

28 Nishtar S. The Gateway paper.Health system in Pakistan-A way forward. Islamabad. Pakistan's Health Policy Forum and Heartfile. 2006. Available from: http://www.heartfile.org/pdf/phpf-GWP.pdf Morsy, A. UN abortion policies. 2009 [cited 2013, March 1]. Available from: http://www.unpost.org/?p=81

30 Government of Pakistan. Reproductive Health Services Package.Ministry of Health and Ministry of Population Welfare. Islamabad. 1999. 
31 Government of Pakistan. Draft Reproductive Health Policy for Pakistan. Planning Division, Islamabad. 2000

32 Shah I, Ahman E. Unsafe abortion in 2008: Global and regional levels and trends. Reproductive Health Matters.2010, November. 18 (36): 90-101.

33 Singh S, Wulf D, Hussain R, Bankole A, Sedgh G. Abortion worldwide: A decade of uneven progress. New York. The Alan Guttmacher Institute. 2009.

34 Sedgh G, Singh S, Shah IH, Ahman E, Henshaw SK, Bankole A. Induced abortion: incidence and trends worldwide from 1995 to 2008. The Lancet.2012, February. 379 (9816): 625-632.

35 Bhutta ZA, Lassi Z, Pariyo G, Huicho L. Global experience of community health workers for delivery of health-related millennium development goals: A systematic review, country case studies, and recommendations for integration into national health systems. World Health Organization. 2010

USAID. Community and Formal Health System Support for Enhanced Community Health Worker Performance: A U.S. Government Evidence Summit, Final Report. 2012, December.

37 Population Reference Bureau. Family Planning Worldwide 2008 Data Sheet. Available from: http://www.prb.org/Publications/Datasheets/2008/familyplanningworldwide.aspx

38 Connor A. Reducing future unintended pregnancies. Marie Stopes International [cited 2013, Feb 26]. Available from: http://www.mariestopes.org/our-impact/section-6/family-planning-following-safeabortion-and-post-abortion-care

39 Peterson LS, Oakley D, Potter LS, Darroch JE. Women's efforts to prevent pregnancy: Consistency of oral contraceptive Use. Family Planning Perspectives. 1998. 30(1): 19-23.

40 Johnson BR, Ndhlovu S, Farr SL, Chipato T. Reducing unplanned pregnancy and abortion in Zimbabwe through post-abortion contraception. Studies in Family Planning.2002, June. 33(2): 195-202.

41 Lule E, Singh S, Chowdhury SA. Fertility regulation behaviours and their costs: Contraception and unintended pregnancies in Africa and Eastern Europe \& Central Asia. Health, Nutrition \& Population Discussion Paper. Washington DC. World Bank. 2007. [pp. 8-39]. Available from: http://siteresources.worldbank.org/HEALTHNUTRITIONANDPOPULATION/Resources/2816271095698140167/FertilityRegulationsFinal.pdf

42 Saleem S, Fikree FF. The quest for small family size among Pakistani women-is voluntary termination of pregnancy a matter of choice or necessity? Journal of the Pakistan Medical Association.2005, July. 55 (7): 288-291.

43 Collumbien M, Busza J, Cleland J and Campbell O..Social science methods for research on sexual and reproductive health. Switzerland. WHO. 2012. Available from:

http://whqlibdoc.who.int/publications/2012/9789241503112_eng.pdf

44 Henshaw SK, Adewole I, Singh S, Bankole A, Oye-Adeniran B, Hussain R. Severity and cost of unsafe abortion complications treated in Nigerian hospitals. International Family Planning Perspectives.2008, March. 34(1): 40-50. 
45 Azmat SK, Shaikh BT, Mustafa G, Hameed W, Bilgrami M. Delivering post-abortion care through a community-based reproductive health volunteer programme in Pakistan. Journal of Biosocial Science. 2012. 44(6): 719-31.

46 Regional Health Systems Observatory EMRO. Health Systems Profile- Pakistan. Health system organization. [cited 2013, May 21]. Available from:

http://gis.emro.who.int/HealthSystemObservatory/PDF/Pakistan/Health\%20system\%20organization. pdf

47 Faúndes A, Santos LC, Carvalho M, Gras C. Post-abortion complications after interruption of pregnancy with misoprostol. Advances in Contraception. 1996, March. 12(1): 1-9.

48 Schenker JG, Margalioth EJ. Intra-uterine adhesions: an updated appraisal. Fertility Sterility. $1982: 37$ (5): 593-610.

49 Kodaman P, Arici A. Intrauterine adhesions and fertility outcome: how to optimize success? Current opinion in Obstetrics and Gynaecology. 2007. 19 (3): 207-214.

50 Buttram VC, Turati G. Uterine synechiae: Variations in severity and some conditions which may be conducive to severe adhesions. International Journal of Fertility. 1977. 22 (2): 98-103.

51 Heikinheimo O, Gissler M, Suhonen S. Age, parity, history of abortion and contraceptive choices affect the risk of repeat abortion. Contraception. 2008. 78(2): 149-154

52 Curtis C, Huber D, Moss-Knight T. Postabortion family planning: addressing the cycle of repeat unintended pregnancy and abortion, International perspectives on sexual and reproductive health. 2010. 36(1):44-48.

53 Pope C, Ziebland S, Mays N. Qualitative research in health care: analysing qualitative data. British Medical Journal. 2000. 320(7227): 114-116. 


\section{Appendices}

\section{Annex 1: Ranking of districts according to income poverty at the district level}

\begin{tabular}{|c|c|c|c|c|c|c|c|c|c|c|c|}
\hline Province & $\begin{array}{l}\text { Colour } \\
\text { code }\end{array}$ & District & $\begin{array}{c}\text { \% of } \\
\text { population } \\
\text { below poverty } \\
\text { line }\end{array}$ & $\begin{array}{c}\text { Provincial } \\
\text { ranking }\end{array}$ & $\begin{array}{l}\text { National } \\
\text { ranking }\end{array}$ & Mapped & $\begin{array}{c}\% \text { of females } \\
\text { aged } 15-49 \\
\text { in total pop. }\end{array}$ & $\begin{array}{c}\text { Females aged } \\
15-49 \text { in total } \\
\text { pop. 2004* }\end{array}$ & $\begin{array}{c}\% \text { of } \\
\text { population } \\
\text { urban }\end{array}$ & $\begin{array}{c}\text { District } \\
\text { population } \\
2004^{*}\end{array}$ & $\begin{array}{l}\text { Sampled for } \\
2002\end{array}$ \\
\hline Sindh & & Karachi & 9.15 & 1 & 1 & Yes & 23.3 & $2,631,968$ & 91.6 & $11,296,000$ & Yes* \\
\hline Sindh & & Hyderabad & 23.1 & 2 & 18 & No & 23.1 & 765,765 & 50.8 & $3,315,000$ & Yes \\
\hline Sindh & & Sanghar & 24.67 & 3 & 21 & Yes & 21.6 & 359,856 & 22.8 & $1,666,000$ & Yes \\
\hline Sindh & & Sukkur & 24.96 & 4 & 22 & Yes & 21.8 & 226,938 & 50.5 & $1,041,000$ & Yes \\
\hline Sindh & & Khairpur & 27.41 & 5 & 27 & Yes & 21.5 & 381,195 & 23.6 & $1,773,000$ & Yes \\
\hline Sindh & & UmerKot & 28.5 & 6 & 31 & Yes & 22 & 228,580 & 33.1 & $1,039,000$ & \\
\hline Sindh & & Tharparkar & 28.9 & 7 & 32 & No & 18.7 & 195,976 & 4.4 & $1,048,000$ & \\
\hline Sindh & & Nawabshah & 32.7 & 8 & 38 & No & 22.6 & 277,528 & 26.4 & $1,228,000$ & Yes \\
\hline Sindh & & NaushahroFeroze & 33.1 & 9 & 39 & No & 22.2 & 276,834 & 17.7 & $1,247,000$ & \\
\hline Sindh & & Jacobabad & 34.16 & 10 & 42 & Yes & 22.5 & 367,650 & 24.4 & $1,634,000$ & \\
\hline Sindh & & Badin & 34.8 & 11 & 45 & No & 21.4 & 278,628 & 16.4 & $1,302,000$ & Yes \\
\hline Sindh & & Dadu & 36.44 & 12 & 49 & Yes & 22.6 & 437,536 & 21.4 & $1,936,000$ & \\
\hline Sindh & & Ghotki & 40.8 & 13 & 58 & Yes & 21.2 & 235,744 & 16.3 & $1,112,000$ & Yes \\
\hline Sindh & & Larkana & 43.33 & 14 & 67 & Yes & 22 & 485,980 & 28.9 & $2,209,000$ & Yes \\
\hline Sindh & & Thatta & 46.87 & 15 & 73 & Yes & 22.2 & 283,272 & 11.2 & $1,276,000$ & \\
\hline Sindh & & Shikarpur & 51.0 & 16 & 80 & Yes & 22.5 & 227,025 & 24.1 & $1,009,000$ & \\
\hline Total Sindh & & & & & & & & & 7660475 & & \\
\hline Punjab & & Rawalpindi & 11.3 & 1 & 2 & No & 24.4 & 931,592 & 53.2 & $3,818,000$ & \\
\hline Punjab & & Lahore & 11.6 & 2 & 3 & Yes & 23.5 & $1,685,185$ & 82.4 & $7,171,000$ & \\
\hline Punjab & & Jhelum & 12.32 & 3 & 4 & Yes & 24 & 255,360 & 27.7 & $1,064,000$ & Yes \\
\hline Punjab & & Gujrat & 12.7 & 4 & 5 & No & 23.4 & 544,050 & 27.7 & $2,325,000$ & \\
\hline Punjab & & Sialkot & 14.0 & 5 & 6 & No & 22.6 & 698,566 & 26.2 & $3,091,000$ & Yes \\
\hline
\end{tabular}




\begin{tabular}{|c|c|c|c|c|c|c|c|c|c|c|c|}
\hline Province & $\begin{array}{l}\text { Colour } \\
\text { code }\end{array}$ & District & $\begin{array}{c}\text { \% of } \\
\text { population } \\
\text { below poverty } \\
\text { line } \\
\end{array}$ & $\begin{array}{l}\text { Provincial } \\
\text { ranking }\end{array}$ & $\begin{array}{c}\text { National } \\
\text { ranking }\end{array}$ & Mapped & $\begin{array}{c}\% \text { of females } \\
\text { aged } 15-49 \\
\text { in total pop. }\end{array}$ & $\begin{array}{c}\text { Females aged } \\
15-49 \text { in total } \\
\text { pop. 2004* }\end{array}$ & $\begin{array}{c}\% \text { of } \\
\text { population } \\
\text { urban }\end{array}$ & $\begin{array}{c}\text { District } \\
\text { population } \\
\text { 2004* }\end{array}$ & $\begin{array}{c}\text { Sampled for } \\
2002\end{array}$ \\
\hline Punjab & & Attock & 14.1 & 6 & 7 & No & 24.1 & 348,727 & 21.3 & $1,447,000$ & Yes \\
\hline Punjab & & MandiBahauddin & 17.3 & 7 & 8 & No & 22.5 & 296,325 & 15.2 & $1,317,000$ & \\
\hline Punjab & & Chakwal & 18.1 & 8 & 9 & No & 25 & 307,500 & 12.2 & $1,230,000$ & \\
\hline Punjab & & Bhakkar & 18.2 & 9 & 10 & No & 21.8 & 260,074 & 16 & $1,193,000$ & Yes \\
\hline Punjab & & Toba Tek Singh & 19.0 & 10 & 11 & No & 22.5 & 414,225 & 18.8 & $1,841,000$ & Yes \\
\hline Punjab & & Gujranwala & 19.0 & 11 & 12 & No & 22 & 849,200 & 50.5 & $3,860,000$ & Yes \\
\hline Punjab & & Narowal & 19.3 & 12 & 13 & No & 22 & 315,920 & 12.2 & $1,436,000$ & Yes \\
\hline Punjab & & Faisalabad & 19.8 & 13 & 14 & No & 22.5 & $1,386,450$ & 42.7 & $6,162,000$ & \\
\hline Punjab & & Sahiwal & 21.7 & 14 & 17 & No & 22.5 & 470,700 & 16.4 & $2,092,000$ & Yes \\
\hline Punjab & & Hafizabad & 24.0 & 15 & 19 & No & 22 & 207,900 & 18.8 & 945,000 & Yes \\
\hline Punjab & & Khushab & 24.4 & 16 & 20 & No & 24 & 246,720 & 25.3 & $1,028,000$ & Yes \\
\hline Punjab & & Sargodha & 25.7 & 17 & 23 & No & 22.7 & 686,902 & 28.1 & $3,026,000$ & \\
\hline Punjab & & Sheikhupura & 26.2 & 18 & 24 & No & 21.5 & 810,335 & 26.2 & $3,769,000$ & \\
\hline Punjab & & Kasur & 28.2 & 19 & 29 & No & 20.7 & 558,072 & 22.8 & $2,696,000$ & Yes \\
\hline Punjab & & Okara & 30.0 & 20 & 34 & No & 21.7 & 549,878 & 23 & $2,534,000$ & Yes \\
\hline Punjab & & Vehari & 30.03 & 21 & 35 & Yes & 21.8 & 517,314 & 16 & $2,373,000$ & \\
\hline Punjab & & Jhang & 32.3 & 22 & 36 & No & 22 & 707,740 & 23.4 & $3,217,000$ & Yes \\
\hline Punjab & & Bahawalnagar & 32.5 & 23 & 37 & No & 22.1 & 517,140 & 19.1 & $2,340,000$ & Yes \\
\hline Punjab & & Mianwali & 35.4 & 24 & 47 & No & 23 & 275,770 & 20.8 & $1,199,000$ & Yes \\
\hline Punjab & & Pakpattan & 36.7 & 25 & 51 & No & 21.9 & 319,740 & 14.2 & $1,460,000$ & \\
\hline Punjab & & Multan & 38.4 & 26 & 53 & Yes & 22 & 778,140 & 42.2 & $3,537,000$ & Yes \\
\hline Punjab & & Khanewal & 38.84 & 27 & 54 & Yes & 22 & 516,560 & 17.6 & $2,348,000$ & Yes \\
\hline Punjab & & Bahawalpur & 39.46 & 28 & 56 & Yes & 21.4 & 590,854 & 27.3 & $2,761,000$ & Yes \\
\hline Punjab & & Leiah & 40.9 & 29 & 60 & No & 21.2 & 269,664 & 13 & $1,272,000$ & Yes \\
\hline Punjab & & Rahim Yar Khan & 45.87 & 30 & 71 & Yes & 21.2 & 755,780 & 19.6 & $3,565,000$ & Yes \\
\hline Punjab & & Lodhran & 48.4 & 31 & 75 & No & 21.1 & 280,630 & 14.5 & $1,330,000$ & \\
\hline Punjab & & Dera Ghazi Khan & 51.01 & 32 & 79 & Yes & 20 & 373,000 & 13.9 & $1,865,000$ & \\
\hline
\end{tabular}




\begin{tabular}{|c|c|c|c|c|c|c|c|c|c|c|c|}
\hline Province & $\begin{array}{l}\text { Colour } \\
\text { code }\end{array}$ & District & $\begin{array}{c}\text { \% of } \\
\text { population } \\
\text { below poverty } \\
\text { line } \\
\end{array}$ & $\begin{array}{c}\text { Provincial } \\
\text { ranking }\end{array}$ & $\begin{array}{c}\text { National } \\
\text { ranking }\end{array}$ & Mapped & $\begin{array}{c}\% \text { of females } \\
\text { aged } 15-49 \\
\text { in total pop. }\end{array}$ & $\begin{array}{c}\text { Females aged } \\
15-49 \text { in total } \\
\text { pop. 2004* }\end{array}$ & $\begin{array}{c}\% \text { of } \\
\text { population } \\
\text { urban }\end{array}$ & $\begin{array}{c}\text { District } \\
\text { population } \\
\text { 2004* }\end{array}$ & $\begin{array}{l}\text { Sampled for } \\
2002\end{array}$ \\
\hline Punjab & & Rajanpur & 54.16 & 33 & 84 & Yes & 20.2 & 253,106 & 14.5 & $1,253,000$ & Yes \\
\hline Punjab & & Muzaffargarh & 56.3 & 34 & 90 & No & 20.3 & 607,376 & 12.9 & $2,992,000$ & Yes \\
\hline Total Punjab & & & & & & & & & 18586495 & & \\
\hline Khyber Pukhtunkhwa & & Mansehra & 20.74 & 1 & 15 & Yes & 23.3 & 309,890 & 5.3 & $1,330,000$ & \\
\hline Khyber Pukhtunkhwa & & Abbottabad & 21.2 & 2 & 16 & No & 23.7 & 240,792 & 17.9 & $1,016,000$ & \\
\hline Khyber Pukhtunkhwa & & Haripur & 27.3 & 3 & 25 & No & 23.8 & 189,924 & 12 & 798,000 & Yes \\
\hline Khyber Pukhtunkhwa & & Swat & 27.3 & 4 & 26 & No & 21 & 304,500 & 13.8 & $1,450,000$ & Yes \\
\hline Khyber Pukhtunkhwa & & Nowshera & 28.0 & 5 & 28 & No & 21.9 & 220,752 & 26 & $1,008,000$ & Yes \\
\hline Khyber Pukhtunkhwa & & Kohat & 28.53 & 6 & 30 & Yes & 22.4 & 145,376 & 27 & 649,000 & \\
\hline Khyber Pukhtunkhwa & & Batagram & 29.22 & 7 & 33 & Yes & 20.6 & 72,924 & 0 & 354,000 & \\
\hline Khyber Pukhtunkhwa & & Bannu & 33.2 & 8 & 40 & No & 21.2 & 165,572 & 7 & 781,000 & \\
\hline Khyber Pukhtunkhwa & & Lower Dir & 34.6 & 9 & 43 & No & 21.2 & 175,536 & 6.2 & 828,000 & \\
\hline Khyber Pukhtunkhwa & & Dera Ismail Khan & 34.6 & 10 & 44 & No & 20.7 & 203,481 & 14.8 & 983,000 & \\
\hline Khyber Pukhtunkhwa & & Tank & 34.9 & 11 & 46 & No & 20.4 & 56,100 & 15 & 275,000 & \\
\hline Khyber Pukhtunkhwa & & Kohistan & 35.6 & 12 & 48 & No & 18.5 & 100,825 & 0 & 545,000 & \\
\hline Khyber Pakhtunkhwa & & Peshawar & 36.5 & 13 & 50 & No & 21.5 & 500,520 & 48.7 & $2,328,000$ & \\
\hline Khyber Pakhtunkhwa & & Karak & 36.9 & 14 & 52 & No & 25 & 124,250 & 6.5 & 497,000 & Yes \\
\hline Khyber Pakhtunkhwa & & Malakand PA & 39.2 & 15 & 55 & No & 20.4 & 106,488 & 9.5 & 522,000 & \\
\hline Khyber Pakhtunkhwa & & Swabi & 39.6 & 16 & 57 & Yes & 22.2 & 262,848 & 17.5 & $1,184,000$ & \\
\hline Khyber Pakhtunkhwa & & Charsadda & 40.83 & 17 & 59 & Yes & 21.1 & 248,769 & 18.9 & $1,179,000$ & \\
\hline Khyber Pakhtunkhwa & & Chitral & 41.0 & 18 & 61 & No & 20.7 & 76,176 & 9.6 & 368,000 & Yes \\
\hline Khyber Pakhtunkhwa & & Mardan & 42.46 & 19 & 65 & Yes & 21.3 & 358,692 & 20.2 & $1,684,000$ & \\
\hline Khyber Pakhtunkhwa & & Hangu & 43.2 & 20 & 66 & No & 22.7 & 82,401 & 20.4 & 363,000 & \\
\hline Khyber Pakhtunkhwa & & Buner & 45.38 & 21 & 69 & Yes & 21.7 & 126,728 & 0 & 584,000 & \\
\hline Khyber Pakhtunkhwa & & LakkiMarwat & 46.5 & 22 & 72 & No & 20.7 & 116,955 & 9.6 & 565,000 & Yes \\
\hline Khyber Pakhtunkhwa & & Shangla & 50.8 & 23 & 77 & No & 20.9 & 104,709 & 0 & 501,000 & \\
\hline
\end{tabular}




\begin{tabular}{|c|c|c|c|c|c|c|c|c|c|c|c|}
\hline Province & $\begin{array}{l}\text { Colour } \\
\text { code }\end{array}$ & District & $\begin{array}{c}\text { \% of } \\
\text { population } \\
\text { below poverty } \\
\text { line } \\
\end{array}$ & $\begin{array}{l}\text { Provincial } \\
\text { ranking }\end{array}$ & $\begin{array}{c}\text { National } \\
\text { ranking }\end{array}$ & Mapped & $\begin{array}{c}\% \text { of females } \\
\text { aged } 15-49 \\
\text { in total pop. }\end{array}$ & $\begin{array}{c}\text { Females aged } \\
15-49 \text { in total } \\
\text { pop. 2004* }\end{array}$ & $\begin{array}{c}\% \text { of } \\
\text { population } \\
\text { urban }\end{array}$ & $\begin{array}{c}\text { District } \\
\text { population } \\
\text { 2004* }\end{array}$ & $\begin{array}{l}\text { Sampled for } \\
2002\end{array}$ \\
\hline Khyber Pakhtunkhwa & & Upper Dir & 54.53 & 24 & 87 & Yes & 19.9 & 132,136 & 4 & 664,000 & \\
\hline Total KPK & & & & & & & & & 4426344 & & \\
\hline Balochistan & & Quetta & 34.2 & 1 & 41 & No & 22.4 & 196,896 & 25.6 & 879,000 & \\
\hline Balochistan & & Ziarat & 41.3 & 2 & 62 & No & 23.6 & 9,204 & 1.9 & 39,000 & \\
\hline Balochistan & & Kalat & 41.9 & 3 & 63 & No & 21.1 & 58,025 & 14.2 & 275,000 & \\
\hline Balochistan & & Mastung & 42.3 & 4 & 64 & No & 21.4 & 40,874 & 14.9 & 191,000 & \\
\hline Balochistan & & Jaffarabad & 44.14 & 5 & 68 & Yes & 22.6 & 113,226 & 19.8 & 501,000 & \\
\hline Balochistan & & Bolan & 45.6 & 6 & 70 & No & 20.9 & 69,597 & 13.7 & 333,000 & \\
\hline Balochistan & & Gawadar & 47.55 & 7 & 74 & Yes & 21.3 & 45,795 & 54 & 215,000 & \\
\hline Balochistan & & Panjgur & 49.7 & 8 & 76 & No & 19.4 & 52,574 & 9.1 & 271,000 & \\
\hline Balochistan & & Khuzdar & 50.96 & 9 & 78 & Yes & 21.1 & 101,913 & 28.3 & 483,000 & \\
\hline Balochistan & & Loralai & 52.1 & 10 & 81 & No & 22.3 & 76,712 & 11.8 & 344,000 & \\
\hline Balochistan & & Barkhan & 52.8 & 11 & 82 & No & 21.6 & 25,920 & 7.4 & 120,000 & \\
\hline Balochistan & & JhalMagsi & 53.4 & 12 & 83 & No & 21.1 & 26,797 & 7.4 & 127,000 & \\
\hline Balochistan & & Musakhel & 54.3 & 13 & 85 & No & 17.5 & 27,125 & 8.6 & 155,000 & \\
\hline Balochistan & & Kech & 54.4 & 14 & 86 & Yes & 22.8 & 108,984 & 16.6 & 478,000 & \\
\hline Balochistan & & Kharan & 55.5 & 15 & 88 & No & 21.6 & 51,624 & 13.4 & 239,000 & Yes \\
\hline Balochistan & & Sibi & 55.8 & 16 & 89 & No & 21.8 & 45,562 & 32.1 & 209,000 & \\
\hline Balochistan & & Nasirabad & 57.3 & 17 & 91 & No & 22.5 & 64,125 & 15.6 & 285,000 & \\
\hline Balochistan & & Killa Abdullah & 58.8 & 18 & 92 & No & 20.5 & 87,740 & 15.3 & 428,000 & \\
\hline Balochistan & & KillaSaifullah & 60.7 & 19 & 93 & No & 20 & 44,800 & 13.1 & 224,000 & \\
\hline Balochistan & & Awaran & 61.5 & 20 & 94 & No & 21.7 & 29,729 & 0 & 137,000 & \\
\hline Balochistan & & Pishin & 62.4 & 21 & 95 & No & 20.6 & 87,550 & 6.3 & 425,000 & Yes \\
\hline Balochistan & & Zhob & 65.99 & 22 & 96 & Yes & 18.3 & 58,194 & 15.9 & 318,000 & \\
\hline Balochistan & & Lasbela & 66.4 & 23 & 97 & Yes & 21.2 & 76,744 & 36.9 & 362,000 & \\
\hline Balochistan & & Chagai & 76.9 & 24 & 98 & No & 19.9 & 46,566 & 17.7 & 234,000 & \\
\hline
\end{tabular}




\begin{tabular}{|c|c|c|c|c|c|c|c|c|c|c|c|}
\hline Province & $\begin{array}{l}\text { Colour } \\
\text { code }\end{array}$ & District & $\begin{array}{c}\text { \% of } \\
\text { population } \\
\text { below poverty } \\
\text { line }\end{array}$ & $\begin{array}{c}\text { Provincial } \\
\text { ranking }\end{array}$ & $\begin{array}{l}\text { National } \\
\text { ranking }\end{array}$ & Mapped & $\begin{array}{c}\% \text { of females } \\
\text { aged } 15-49 \\
\text { in total pop. }\end{array}$ & $\begin{array}{c}\text { Females aged } \\
15-49 \text { in total } \\
\text { pop. 2004* }\end{array}$ & $\begin{array}{c}\% \text { of } \\
\text { population } \\
\text { urban }\end{array}$ & $\begin{array}{c}\text { District } \\
\text { population } \\
\text { 2004* }\end{array}$ & $\begin{array}{c}\text { Sampled for } \\
2002\end{array}$ \\
\hline Balochistan & & DeraBugti & \multicolumn{3}{|c|}{ Survey Not Conducted } & No & 21 & 44,100 & 8.5 & 210,000 & \\
\hline Balochistan & & Kohlu & \multicolumn{3}{|c|}{ Survey Not Conducted } & No & 22.4 & 25,760 & 9.7 & 115,000 & \\
\hline Total Balochistan & & & & & & & & & $1,616,136$ & & \\
\hline Total National & & & & & & & & $32,289,450$ & & $145,741,000$ & \\
\hline
\end{tabular}

*Population is projected for the year 2004 by using Pakistan Population Data Sheet -2001 (NIPS)

\section{Colour Codes}

$\begin{array}{ll}\text { Sampled Districts } & 24 \\ \text { Teaching Hospitals } & 11 \\ \text { Overlapping (Sampled + Teaching Hospitals } & 3 \\ \text { GIS Mapping available } & 34 \\ \text { 2002 Sampled Districts } & 39 \\ \text { Mapping Required } & 4\end{array}$


Annex 2: Sociall and health indicators of study districts

\begin{tabular}{|c|c|c|c|c|c|c|c|c|c|c|c|c|c|c|}
\hline $\begin{array}{l}\text { Prov. } \\
\text { Name }\end{array}$ & $\begin{array}{c}\text { District } \\
\text { Name }\end{array}$ & $\begin{array}{c}\% \text { People } \\
\text { below } \\
\text { Poverty } \\
\text { Line }\end{array}$ & $\begin{array}{l}\text { Provincial } \\
\text { Ranking }\end{array}$ & $\begin{array}{l}\text { National } \\
\text { Ranking }\end{array}$ & $\begin{array}{c}\text { Females age } \\
15-49 \text { in total } \\
\text { pop. } 2004 \\
\end{array}$ & $\begin{array}{l}\text { \% of } \\
\text { urban } \\
\text { Pop. }\end{array}$ & $\begin{array}{l}\text { District } \\
\text { Population } \\
2004 \\
\end{array}$ & \begin{tabular}{|l} 
Females age \\
$15-49$ in total \\
pop. 2011* \\
\end{tabular} & \begin{tabular}{|c|}
$\begin{array}{c}\text { District } \\
\text { Population } \\
2011\end{array}$ \\
\end{tabular} & $\begin{array}{c}\text { Sex Ratio } \\
\text { (males per } \\
100 \\
\text { females) } \\
\end{array}$ & $\begin{array}{l}\text { Pop. Density } \\
\text { Per SQ.KM. }\end{array}$ & \begin{tabular}{|c} 
Average \\
Househol \\
d Size
\end{tabular} & $\begin{array}{c}\text { Literacy } \\
\text { Ratio } \\
(10+) \\
\end{array}$ & \begin{tabular}{|c|c} 
Contraceptive \\
Prevalence \\
Rate (\%)
\end{tabular} \\
\hline \multirow{11}{*}{ Sindh } & Dadu & 36.44 & 12 & 49 & 437,536 & 21.4 & $1,936,000$ & \begin{tabular}{|l|}
491550 \\
\end{tabular} & 2175000 & 110.6 & \begin{tabular}{|r|}
88.6 \\
\end{tabular} & 5.5 & 35.6 & $\begin{array}{r}16.3 \\
\end{array}$ \\
\hline & Hyderabad & 23.1 & 2 & 18 & 765,765 & 50.8 & $3,315,000$ & 860244 & 3724000 & 109.5 & 523.9 & 6.0 & 44.3 & 34.6 \\
\hline & Jacobabad & 34.16 & 10 & 42 & 367,650 & 24.4 & $1,634,000$ & 413100 & 1836000 & 109.2 & 270.1 & 5.6 & 23.7 & 14.7 \\
\hline & Karachi & 9.15 & 1 & 1 & $2,631,968$ & 91.6 & $11,296,000$ & 2957003 & 12691000 & 118.1 & 13954.4 & 6.8 & 76.0 & 51.6 \\
\hline & Khairpur & 27.41 & 5 & 27 & 381,195 & 23.6 & $1,773,000$ & 428280 & 1992000 & 110.1 & 97.2 & 6.0 & 35.5 & 19.9 \\
\hline & Larkana & 43.33 & 14 & 67 & 485,980 & 28.9 & $2,209,000$ & 546040 & 2482000 & 106.4 & 259.6 & 5.9 & 35.0 & 14.0 \\
\hline & Nawabshah & 32.7 & 8 & 38 & 277,528 & 26.4 & $1,228,000$ & 311880 & 1380000 & 107.7 & 238 & 6.0 & 34.1 & 15.4 \\
\hline & Sanghar & 24.67 & 3 & 21 & 359,856 & 22.8 & $1,666,000$ & 404352 & 1872000 & 110.4 & 135.4 & 6.4 & 30.9 & 15.1 \\
\hline & Sukkur & 24.96 & 4 & 22 & 226,938 & 50.5 & $1,041,000$ & 255060 & 1170000 & 113.7 & 175.9 & 6.5 & 46.6 & 24.5 \\
\hline & Thatta & 46.87 & 15 & 73 & 283,272 & 11.2 & $1,276,000$ & 318348 & 1434000 & 112.5 & 64.1 & 5.1 & 22.1 & 20.3 \\
\hline & Umerkot & 28.5 & 6 & 31 & 228,580 & 33.1 & $1,039,000$ & 256740 & 1167000 & 111.6 & 118.2 & 5.4 & 24.8 & 15.0 \\
\hline \multirow{16}{*}{ Punjab } & Attock & 14.1 & 6 & 7 & 348,727 & 21.3 & $1,447,000$ & 390179 & 1619000 & 99.6 & 186 & 6.1 & 49.3 & 19.0 \\
\hline & hawalpur & 39.46 & 28 & 56 & 590,854 & 27.3 & $2,761,000$ & 661046 & 3089000 & 110.8 & 98 & 6.8 & 34.7 & 25.5 \\
\hline & Faisalabad & 19.8 & 13 & 14 & $1,386,450$ & 42.7 & $6,162,000$ & 1550925 & 6893000 & 108.6 & 927.2 & 7.2 & 52.0 & 36.0 \\
\hline & Gujrat & 12.7 & 4 & 5 & 544,050 & 27.7 & $2,325,000$ & 608634 & 2601000 & 100.4 & 641.6 & 6.6 & 62.2 & 40.0 \\
\hline & Hafizabad & 24.0 & 15 & 19 & 207,900 & 18.8 & 945,000 & 232760 & 1058000 & 108.4 & 351.9 & 7.1 & 50.5 & 25.9 \\
\hline & Jhelum & 12.32 & 3 & 4 & 255,360 & 27.7 & $1,064,000$ & 285600 & 1190000 & 99.8 & 261.2 & 6.1 & 68.9 & 39.0 \\
\hline & Khanewal & 38.84 & 27 & 54 & 516,560 & 17.6 & $2,348,000$ & 577720 & 2626000 & 107.7 & 475.6 & 7.0 & 40.0 & 18.7 \\
\hline & Lahore & 11.6 & 2 & 3 & $1,685,185$ & 82.4 & $7,171,000$ & 1885170 & 8022000 & 111.3 & 3565.9 & 7.1 & 64.6 & 53.0 \\
\hline & Mianwali & 35.4 & 24 & 47 & 275,770 & 20.8 & $1,199,000$ & 308660 & 1342000 & 100.8 & 180.9 & 7.1 & 42.8 & 20.2 \\
\hline & Multan & 38.4 & 26 & 53 & 778,140 & 42.2 & $3,537,000$ & 870540 & 3957000 & 110.4 & 837.9 & 7.1 & 43.4 & 33.0 \\
\hline & Narowal & 19.3 & 12 & 13 & 315,920 & 12.2 & $1,436,000$ & 353320 & 1606000 & 101.2 & 541.3 & 7.4 & 52.7 & 38.7 \\
\hline & Rahim Yar Khan & 45.87 & 30 & 71 & 755,780 & 19.6 & $3,565,000$ & 845456 & 3988000 & 108.8 & 264.4 & 7.5 & 33.1 & 19.2 \\
\hline & Rajanpur & 54.16 & 33 & 84 & 253,106 & 14.5 & $1,253,000$ & 283002 & 1401000 & 111.1 & 89.6 & 7.3 & 20.7 & 11.8 \\
\hline & Rawalpindi & 11.3 & 1 & 2 & 931,592 & 53.2 & $3,818,000$ & 1042124 & 4271000 & 104.9 & 636.5 & 6.4 & 70.4 & 41.6 \\
\hline & Vehari & 30.03 & 21 & 35 & 517,314 & 16 & $2,373,000$ & 578572 & 2654000 & 107.7 & 479 & 6.9 & 36.8 & 22.0 \\
\hline & Islamabad & & - & - & & & & & & 117.0 & 888.8 & 6.2 & 72.4 & 49.3 \\
\hline \multirow{5}{*}{$\begin{array}{c}\text { Khyber } \\
\text { Pakhtunkhwa }\end{array}$} & Abbottabad & 21.2 & 2 & 16 & 240,792 & 17.9 & $1,016,000$ & 277527 & 1171000 & 100.2 & 447.7 & 6.4 & 56.6 & 29.1 \\
\hline & Mansehra & 20.74 & 1 & 15 & 309,890 & 5.3 & $1,330,000$ & 357189 & 1533000 & 98.4 & 252 & 6.7 & 36.3 & 30.0 \\
\hline & Mardan & 42.46 & 19 & 65 & 358,692 & 20.2 & $1,684,000$ & 413220 & 1940000 & 106.6 & 894.7 & 8.4 & 36.5 & 15.2 \\
\hline & Peshawar & 36.5 & 13 & 50 & 500,520 & $\begin{array}{l}48.7 \\
\end{array}$ & $2,328,000$ & 576845 & 2683000 & 110.8 & 1606.3 & 8.5 & 41.8 & 35.9 \\
\hline & Swabi & 39.6 & 16 & 57 & 262,848 & 17.5 & $1,184,000$ & 303030 & 1365000 & 101.2 & 66 & 7.7 & 36.0 & 24.6 \\
\hline \multirow{4}{*}{ Balochistan } & Gawadar & 47.55 & 7 & 74 & 45,795 & 54 & 215,000 & 53463 & 251000 & 115.5 & 14.7 & 5.5 & 25.5 & 21.4 \\
\hline & Jaffarabad & 44.14 & 5 & 68 & 113,226 & 19.8 & 501,000 & 132210 & 585000 & 108.3 & 177 & 7.1 & 18.5 & 13.5 \\
\hline & Lasbella & 66.4 & 23 & 97 & 76,744 & 36.9 & 362,000 & 89464 & 422000 & 115.3 & 20.6 & 6.2 & 22.3 & 30.5 \\
\hline & Quetta & 34.2 & 1 & 41 & 196,896 & 25.6 & 879,000 & 229824 & 1026000 & 118.5 & 286.4 & 8.5 & 57.1 & 33.2 \\
\hline
\end{tabular}

Source: Population data sheet 1998 Census. NIPS 


\section{Annex 3: Members of the technical advisory group and study team}

\begin{tabular}{|c|c|}
\hline $\begin{array}{l}\text { Ahsan,Azra } \\
\text { Technical Consultant } \\
\text { National Committee for Maternal \& Neonatal Health } \\
\text { (NCMNH) } \\
\text { 36-C, 14th Street, Off Khayaban-e-Shamsheer, Phase } \\
\text { 5, DHA / Res: F-71, Block 4, Clifton, Karachi } \\
\text { 021-5341597-8 }\end{array}$ & $\begin{array}{l}\text { Bilgrami,Mohsina } \\
\text { Managing Director } \\
\text { Marie Stopes Society } \\
\text { 21-C, Commercial Area, Old Sunset Boulevard, } \\
\text { DHA-II, Karachi } \\
021-35389125\end{array}$ \\
\hline $\begin{array}{l}\text { Chaudhry, Rizwana } \\
\text { Prof. of Gynaecology \& Obstetrics Rawalpindi } \\
\text { Medical College Rawalpindi } \\
\text { Holy Family Hospital, Rawalpindi } \\
\text { Department of Gynaecology and Obstetrics, Holy } \\
\text { Family Hospital , Rawalpindi } \\
051-9290328-30\end{array}$ & $\begin{array}{l}\text { Hassan,Lubna } \\
\text { President } \\
\text { Society Of Obstetricians And Gynaecologists Of } \\
\text { Pakistan(SOGP) } \\
\text { Peshawar }\end{array}$ \\
\hline $\begin{array}{l}\text { Kamal, Imtiaz } \\
\text { President } \\
\text { Midwifery Association of Pakistan } \\
\text { 36-C, 14th Street, Off Khayaban-e-Shamsheer, Phase } \\
\text { 5, DHA, Karachi } \\
\text { 021-35341597-8 }\end{array}$ & $\begin{array}{l}\text { Mazhar, Amina } \\
\text { Country Manager } \\
\text { Ipas-Pakistan } \\
\text { 36-C, 14th Street, Off Khayaban-e-Shamsheer, Phase } \\
\text { 5, DHA, Karachi } \\
021.32043830\end{array}$ \\
\hline $\begin{array}{l}\text { Mehmood, Ghazala } \\
\text { Gynaecologist \& Obstetrician } \\
\text { Islamabad } \\
\text { G-8/3 / House No. 22, Luqman Hakeem Road, G-6/3, } \\
\text { Islamabad051-9261273 }\end{array}$ & $\begin{array}{l}\text { Mir, Ali Mohammad } \\
\text { Director Programs } \\
\text { Population Council } \\
\text { House No 7, St. 62, F-6/3, Islamabad } \\
051-8445566\end{array}$ \\
\hline $\begin{array}{l}\text { Qazi, YasmeenSabeeh } \\
\text { Senior Country Advisor } \\
\text { The David and Lucile Packard Foundation } \\
\text { 28-C, Second Floor, Rahat Commercial Lane 2, Phase } \\
\text { V1, DHA, Karachi, } 75500 . \\
\text { Tel: } 021-35856906 \text { Fax:021-35856908 }\end{array}$ & $\begin{array}{l}\text { Ruby, Nasreen } \\
\text { Obstetrician and Gynaecologist } \\
\text { H.178, H-1, Street 10, Phase III Hayatabad, } \\
\text { Peshawar }\end{array}$ \\
\hline $\begin{array}{l}\text { Siddiqa, Aisha } \\
\text { Vice President -SOGP, Provincial coordinator- } \\
\text { NCMNH, Faculty member- CPSP. } \\
\text { Obstetrician \& Gynaecologist } \\
\text { Health Deptt. Balochistan } \\
\text { Sandeman Provincial Hospital, Quetta } \\
\text { Fax: 081-9202639 }\end{array}$ & $\begin{array}{l}\text { Shah, Nighat } \\
\text { Gen. Secretary } \\
\text { Society Of Obstetricians And Gynaecologists Of } \\
\text { Pakistan(SOGP) } \\
\text { Jinnah Postgraduate Medical Centre, Karachi } \\
\text { Tel: } 02199205040 \text {, Fax: } 02199205040\end{array}$ \\
\hline
\end{tabular}




\begin{tabular}{|c|c|}
\hline $\begin{array}{l}\text { Tasneem, Nasira } \\
\text { Associate Professor of Gynaecolgy and Obstetrics } \\
\text { Pakistan Institute of Medical Sciences (PIMS ) } \\
\text { MCH Centre PIMS, G-8, Islamabad } \\
051-9261273\end{array}$ & $\begin{array}{l}\text { Zaidi, Shahida } \\
\text { Vice President } \\
\text { FIGO } \\
\text { Initiative for the Prevention of Unsafe Abortion and } \\
\text { its Complications } \\
140 \text { R, Block II, PECHS, Off Sir Syed Road, Karachi } \\
021-4551445,4552129\end{array}$ \\
\hline $\begin{array}{l}\text { Zaman, Farrukh } \\
\text { Principal, } \\
\text { Prof. of Gynaecology \& Obstetrics } \\
\text { Rashid Latif Medical College, Lahore } \\
\text { HameedLatif Hospital, } 14 \text { Abu Bakker block, new } \\
\text { Garden Town, Lahore }\end{array}$ & \\
\hline
\end{tabular}

\section{Study Team:}

\begin{tabular}{|c|c|}
\hline Sathar,Zeba A & Singh,Susheela \\
\hline Country Director & Vice President for Research \\
\hline Population Council & The Alan Guttmacher Institute \\
\hline House No 7, St. 62, F-6/3, Islamabad & 125 Maiden Lane, 7th Floor, New York 10038 USA \\
\hline 051-8445566 & 212-248-1111 Ext. 2264 \\
\hline Jafarey,Sadiqua $\mathbf{N}$ & Shah, Zakir Hussain \\
\hline President & Senior Program Manager \\
\hline National Committee for Maternal \& Neonatal & Population Council \\
\hline Health (NCMNH) & House No 7, St. 62, F-6/3, Islamabad \\
\hline 36-C, Street 14, Off Khayaban-e-Shamsheer, & $051-8445566$ \\
\hline Phase V, D.H.A.Karachi-75500 & \\
\hline $021-35341597-8$ & \\
\hline \multicolumn{2}{|l|}{ Rashida,Gul } \\
\hline \multicolumn{2}{|l|}{ Senior Director Trainings } \\
\hline \multicolumn{2}{|l|}{ Population Council } \\
\hline \multicolumn{2}{|l|}{ House No 7, St. 62, F-6/3, Islamabad } \\
\hline 051-8445566 & \\
\hline
\end{tabular}




\section{Annex 4: Data collection teams}

\section{Quantitative component}

\section{Punjab \\ Sindh}

Dr.Sadaf Gul (Team Leader)

Dr. Muhammad Saleem Shaikh (Team Leader)

Mr.Mumraiz Khan

Dr.SaminaAsif

Mr.HyderSafderAbbasi

Ms.ShabanaArif

Ms.FarzanaArif

Dr.GhaffarBhatti

Dr.Fahadul Hassan

Ms.Rukhsana Hussain

Mr.Zeshan Ali Khan

Dr.GhulamMurtaza

Ms.NaziaMehmood

Dr. Ruby Tariq Shah

Ms.IrfanaShafi

Ms.Shumaila

Ms. Maria Zain

\section{Khyber Pakhtunkhwa}

\section{Balochistan}

Ms.LubnaMehmood (Team Leader)

Mr. Khan Mohammad (Team Leader)

Dr. Abdul Jalal

Dr.Naveed

Ms.ZubaidaKhanum

Dr.Noroz

Dr.Asif Nawaz

\section{Qualitative component}

\section{Supervisor: Dr. Zakir Hussain Shah}

Ms.Iram Kamran

Ms.ZebaTasneem

Mr.Mumraiz Khan

Ms.KanwalEshai

Ms. Ayesha Shafique

Ms.FakhiraRasheed
Mr.Haleem Bhutto

Mr.HaiderSafdarAbbasi

Ms.ShamimLatif

Ms.LubnaMehmood

Ms.FarzanaParveen

Ms. Nadia Abid Khan 


\section{Annex 5a: Health facility statistics on post-abortion care}

Table A1: Health facility statistics on post-abortion care

\begin{tabular}{lcc} 
Data recording & $\%$ & N \\
\hline Record statistics & 82 & 218 \\
Do not record & 18 & 48 \\
\hline Total & 100 & 266 \\
\hline Among those who record statistics & & \\
\hline Recording of statistics is complete & 67 & 147 \\
Missing data(partial recording) & 33 & 71 \\
\hline Number of facilities visited & 100 & 218 \\
\hline
\end{tabular}

\section{Annex 5b: Number of facilities recording statistics on post- abortion complications}

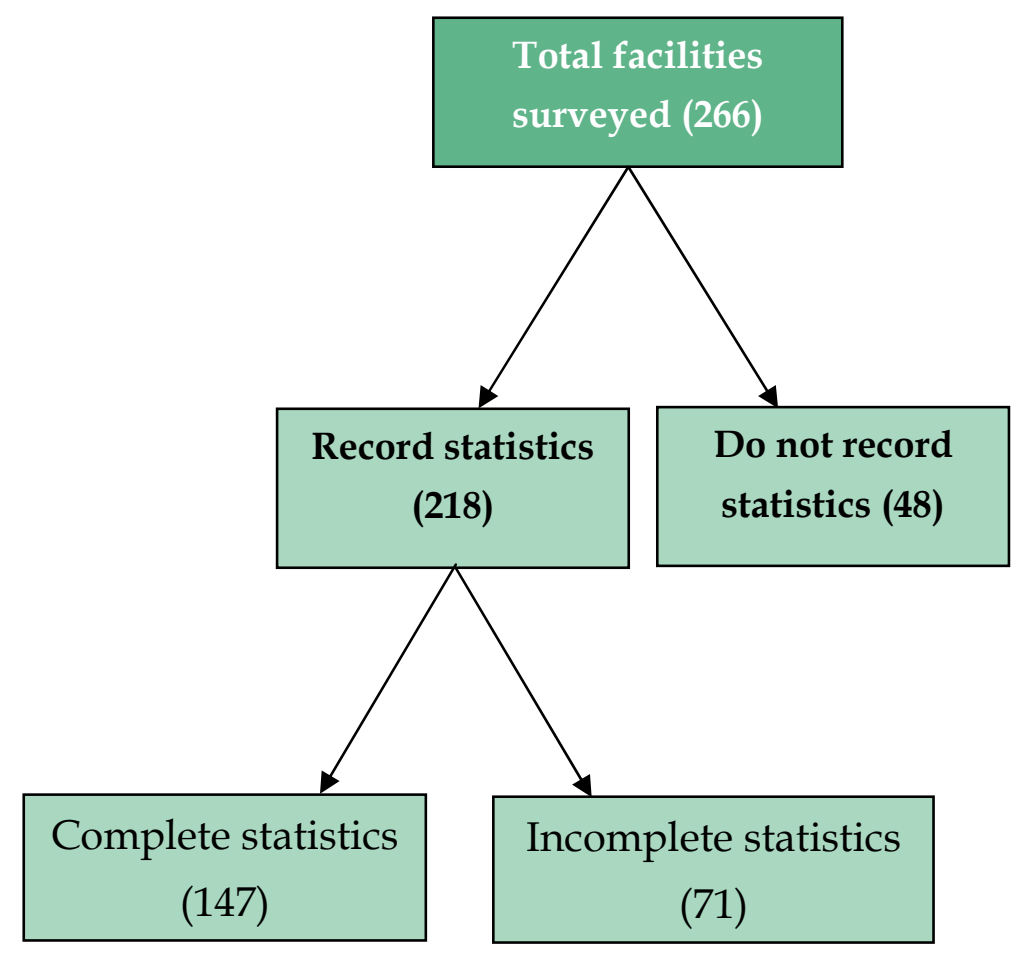




\section{Annex 6: Annual caseload of post-abortion complicationsby}

\section{sector, type of facility and province}

Table A2: Annual caseload* of post-abortion complications (out-and in-patients) by sector, type of facility and province, 2012(hospitals statistics)

\begin{tabular}{|c|c|c|c|c|c|c|c|c|c|c|c|}
\hline \multirow[b]{3}{*}{ Caseload of PAC as: } & \multicolumn{11}{|c|}{ Type of facilities } \\
\hline & \multicolumn{4}{|c|}{ Public } & \multicolumn{4}{|c|}{ Private } & \multicolumn{2}{|c|}{ Overall } & \multirow[b]{2}{*}{ Total } \\
\hline & Teaching & DHQ & THQ & RHC & Teaching & Large* & Medium* & Small* & Public & Private & \\
\hline \multicolumn{12}{|l|}{ Punjab } \\
\hline $\begin{array}{l}\text { Mean \# Out-patients } \\
\text { per facility }\end{array}$ & 1,043 & 506 & 446 & 103 & 414 & 1,000 & 327 & 405 & 217 & 444 & 343 \\
\hline $\begin{array}{l}\text { Mean \# In-patient } \\
\text { per facility }\end{array}$ & 853 & 318 & 95 & 42 & 304 & 325 & 135 & 88 & 105 & 123 & 115 \\
\hline $\begin{array}{l}\text { Mean \# Out- and in- } \\
\text { patient per facility }\end{array}$ & 1,766 & 711 & 518 & 145 & 718 & 1,325 & 462 & 475 & 315 & 553 & 447 \\
\hline \multicolumn{12}{|l|}{ Sindh } \\
\hline $\begin{array}{l}\text { Mean \# Out-patients } \\
\text { per facility }\end{array}$ & 2,101 & 585 & 160 & 128 & 230 & 600 & 123 & 128 & 261 & 158 & 212 \\
\hline $\begin{array}{l}\text { Mean \# In-patient } \\
\text { per facility }\end{array}$ & 815 & 301 & 83 & 31 & 177 & 360 & 94 & 86 & 98 & 107 & 102 \\
\hline $\begin{array}{l}\text { Mean \# Out- and in- } \\
\text { patient per facility }\end{array}$ & 2,916 & 886 & 243 & 159 & 384 & 960 & 217 & 214 & 359 & 264 & 314 \\
\hline \multicolumn{12}{|l|}{ KPK } \\
\hline $\begin{array}{l}\text { Mean \# Out-patients } \\
\text { per facility }\end{array}$ & 394 & 930 & 30 & 117 & 162 & $U$ & 0 & 0 & 307 & 20 & 251 \\
\hline $\begin{array}{l}\text { Mean \# In-patient } \\
\text { per facility }\end{array}$ & 670 & 600 & 23 & 1 & 141 & $U$ & 650 & 0 & 266 & 133 & 234 \\
\hline $\begin{array}{l}\text { Mean \# Out- and in- } \\
\text { patient per facility }\end{array}$ & 1,064 & 1,220 & 38 & 117 & 249 & $\mathbf{U}$ & 650 & 0 & 452 & 149 & 392 \\
\hline \multicolumn{12}{|l|}{ Balochistan } \\
\hline $\begin{array}{l}\text { Mean \# Out-patients } \\
\text { per facility }\end{array}$ & 746 & 152 & 287 & 49 & $U$ & 432 & $U$ & 144 & 109 & 148 & 125 \\
\hline $\begin{array}{l}\text { Mean \# In-patient } \\
\text { per facility }\end{array}$ & 552 & 60 & 70 & 31 & $U$ & 45 & $U$ & 44 & 55 & 44 & 49 \\
\hline $\begin{array}{l}\text { Mean \# Out- and in- } \\
\text { patient per facility }\end{array}$ & 1,298 & 161 & 357 & 76 & $\mathbf{U}$ & 477 & $\mathbf{U}$ & 152 & 148 & 155 & 151 \\
\hline \multicolumn{12}{|l|}{ Pakistan } \\
\hline $\begin{array}{l}\text { Mean \# Out-patients } \\
\text { per facility }\end{array}$ & 1,232 & 491 & 304 & 103 & 294 & 925 & 284 & 295 & 222 & 340 & 280 \\
\hline $\begin{array}{l}\text { Mean \# In-patient } \\
\text { per facility }\end{array}$ & 795 & 288 & 80 & 36 & 216 & 325 & 141 & 78 & 111 & 110 & 110 \\
\hline $\begin{array}{l}\text { Mean \# Out- and in- } \\
\text { patient per facility }\end{array}$ & 1,950 & 650 & 354 & 137 & 481 & 1250 & 417 & 353 & 317 & 431 & 374 \\
\hline
\end{tabular}

Source: Health Facilities Survey, weighted result.

Note: There are no private teaching hospitals and private medium-sized hospitals in Balochistan and no private large hospitals in KPK.

$\mathrm{U}=$ unavailable.

*Private hospitals based on bed size: Small $=5-19$, Medium $=20-80$, Large $=>80$. 


\section{Annex 7: Application of weights to data obtained from public- and private-sectorhealth facilities}

Since this study aimedatpresenting a national snapshot of post-abortion care it was essential to derive weights so that the sample findings could be converted into national estimates of numbers of complications and the quality of care available. In the 2012 Health Facilities Survey, we sampled and collected data from 266 public and private health facilities. To derive national-level estimates on various measurements (including number of post-abortion complications), we applied appropriate weights for various types of public and private health facilities.

\section{Weights for public-sector health facilities:}

Since we had a full count of facilities in the public sector, including bed sizes, we drew a stratified systematic randomsample to select a nationally representative sample of public-sector facilities that provide post-abortion care. This sample was drawn from a master list of the HMIS (Health Management Information System) of public-sector health facilities, obtained from the Provincial Health Departments. All public teaching hospitals, affiliated with medical colleges established before 2011 and providing GYN/OBS services were selected for the study. However, three of them could not be surveyed and weights were applied subsequently to account for their exclusion. Weights were not required for public teaching hospitals since thesewere all included in the study. Among the remaining public facilitieswe sampled approximately $25 \%$ of DHQs, $27 \%$ of THQs and $11 \%$ of RHCs. These proportions were adequate to represent variation in each of these types of facilities, and to be weighted to represent the universe of these facilities. A total of 164 publicsector facilities were surveyed (including teaching hospitals, DHQ, THQ and RHC facilities).

The HMIS listings of public-sector facilities show their number and bed size. Weights $(\mathrm{w})$ were calculated for each level of facility, separately for each of the four provinces, based on bed sizes of facilities. Initially weights were obtained dividing the total number of facilities of each type available in the province $(N)$ by the number of facilities surveyed $(n)$ i.e. by formula $W=N / n$. Similarly, weights were calculated by dividing the total number of beds in each facility type available in each province by the number of beds calculated through the Health Facility Survey. Finally, the weights obtained through bed-size estimation were used to show the nationwidepublic-sector data. 


\section{Weights for private-sector health facilities}

A total of 102 private-sector facilities were surveyed sampled (21 teaching hospitals and 81large, medium-sized and small hospitals). All major private teaching hospitals were included (a complete list of these hospitals was obtained from PMDC). However, certain private teaching hospitals were excluded: those which had less than 50 beds, those that did not provide GYN/OBS services and those which were affiliated with medical colleges that were established after the year 2008. Moreover, a few of the hospitals could not be surveyed due to a variety of reasons (refusals, security reasons etc.). To account for "missing" hospitals weights were applied according to bed size. However, for the other types of private-sector facilities we used a complete listing of such facilities in the 24 districts* (distributed across the four provinces included in the study, and available from a prior Population Council full Census of health facilities), to draw a sample of private-sector facilities that was representative of facilities in these districts.

For thelarge, medium-sized and small hospitals(based on bed sizes), theprivate health facilities enumerated in the 24 sample districtswere used to derive the national and provincial counts of private sector facilities.

To derive weights for private-sector health facilities we followed these steps:

1. Used the 24 districtsCensus as the universe of private facilities.

2. Computed the ratio of public/private beds sizes for these 24 districts.

3. Utilized the full list of facilities in the public sector and applied the public/private ratios of bed size.

4. Applied the information on the proportion of facilities delivering PAC by bed size to the number of facilities.

\footnotetext{
* Data from 20 GIS districts were identical in the HFS and the GIS survey. For another four districts in which GIS data was not available, we conducted a rapid survey to list all private facilities according to the size criteria selected.
} 
Table A3: Weights of health facilities by sector and by number of beds

\begin{tabular}{|c|c|c|c|c|c|c|c|}
\hline \multirow{2}{*}{ Provinces } & \multirow{2}{*}{ Facilities } & \multicolumn{3}{|c|}{ Number of beds (Public)* } & \multicolumn{3}{|c|}{ Number of beds (Private) ${ }^{* *}$} \\
\hline & & $\mathbf{N}$ & $\mathbf{n}$ & Weights & $\mathbf{N}$ & $\mathbf{n}$ & Weights \\
\hline \multirow{4}{*}{ Punjab } & Teaching Hospital & 14,084 & 12,884 & 1.1 & 3,830 & 2,514 & 1.5 \\
\hline & $\mathrm{DHQ}$ & 7,802 & 2,350 & 3.3 & 7,503 & 320 & 23.5 \\
\hline & THQ & 4,675 & 1,294 & 3.6 & 6,653 & 179 & 37.2 \\
\hline & $\mathrm{RHC}$ & 5,518 & 514 & 10.7 & 9,099 & 420 & 15.2 \\
\hline \multirow{4}{*}{ Sindh } & Teaching Hospital & 7,960 & 7,960 & 1.0 & 5,082 & 3,445 & 1.5 \\
\hline & $\mathrm{DHQ}$ & 2,271 & 1,536 & 1.5 & 2,183 & 200 & 10.9 \\
\hline & THQ & 1,553 & 537 & 2.9 & 2,424 & 247 & 9.8 \\
\hline & $\mathrm{RHC}$ & 1,610 & 194 & 8.3 & 3,282 & 126 & 18.2 \\
\hline \multirow{4}{*}{ KPK } & Teaching Hospital & 6,047 & 5,350 & 1.1 & 1,865 & 1,100 & 1.7 \\
\hline & $\mathrm{DHQ}$ & 4,310 & 530 & 8.1 & - & - & 0.0 \\
\hline & THQ & 1,784 & 354 & 5.0 & 225 & 57 & 3.9 \\
\hline & $\mathrm{RHC}$ & 1,435 & 140 & 10.3 & 786 & 36 & 15.3 \\
\hline \multirow{4}{*}{ Balochistan } & Teaching Hospital & 1,960 & 1,960 & 1.0 & - & - & 0.0 \\
\hline & $\mathrm{DHQ}$ & 1,238 & 98 & 12.6 & - & - & 0.0 \\
\hline & THQ & 370 & 120 & 3.1 & 189 & 75 & 2.5 \\
\hline & $\mathrm{RHC}$ & 939 & 95 & 9.9 & 1,382 & 40 & 24.2 \\
\hline
\end{tabular}

*Health Management Information System (HMIS), provincial health departments of Pakistan 2011.

**Mapping of Health and Reproductive Health Services- Survey of Service Delivery Points. Population Council. 2010

We had to make some final adjustments to the weights by assuming that the proportions of smaller facilities saying they were delivering PAC services and not actually equipped to do so was different. We further assumed that about half of all the private facilities saying they were delivering PAC services were doing so at the RHC (small bed-size) level.

We also conducted a small-scale study in two districts to measure the "efficiency" of the private health sector in providing PAC service. The results show that RHC-level private facilities were providing $30-35 \%$ of all PAC services in those districts. These results support thatsimilar assumption made from using GIS mapping data. 


\section{Annex 8: Sampling methodology for 2002 study}

\section{Health facility survey}

The sample included government health facilities ranging from teaching hospitals, District Headquarters Hospitals (DHQ), Tehsil Headquarters Hospitals (THQ), Rural Health Centres (RHC) and Basic Health Units (BHU). The target sample size was 200 facilities based on the assumption that this would provide a reasonable and defensible coverage of all public and private facilities managing post-abortion complications (PAC). Other assumptions included:

A sampling strategy proportional to bed size was utilized to enable a holistic representation of primary and secondary levels of public and private health facilities that are accessed for PAC resulting in the following distribution by facility level:

- Teaching and tertiary hospitals $\quad 25 \%$ (public and private)

- Primary and Secondary facilities $50 \%$ (public)

- Primary and Secondary facilities $25 \%$ (private)

The facility level, primary, secondary and tertiary/university, distribution was disaggregated by catchment's population and type of services provided for public facility and by bed size for private facilities according to the following definition:

- $\quad$ Bed size $\geq 100$

- $\quad$ Bed size $30-99$

- $\quad$ Bed size 6- 29

- $\quad$ Bed size $\leq 5$
DHQ hospital/Private Hospital

THQ Hospital/Private Hospital/maternity home

RHC/Private Hospital/maternity home

BHU/Private Hospital/maternity home

A total of 230 facilities including 52 public and private teaching facilities were included in the study. Of the 230 facilities, 146 were public and the remaining 84 facilities were private. A computerized database, Health Information Management System (HMIS) and the Pakistan Health and Population Welfare Facilities Atlas*, for all public health facilities disaggregated by province and facility level (DHQ, THQ, RHC and BHU) was acquired from the Ministry of Health. The HMIS database served as the sampling universe for the randomized selection of various levels of health facility (DHQ, THQ, RHC, BHU) based on the sampling strategy mentioned earlier.

\footnotetext{
*Center for Research on Poverty Reduction and Income Distribution. 2002. Pakistan Health and Population Welfare Facilities Atlas. Planning Commission: Islamabad
} 


\section{Health professionals survey}

- Survey methodology

Research team members contacted all potential participants in the study, explained the aims of goals of the study and obtained informed consent to participate. Health professionals were asked to respond to questions based on their professional experience and not restricted to perceptions based on the catchment's population where they currently serve. A number of the questions were specifically targeted to eliciting information for differentiating between urban and rural, and poor and non-poor. For example, health professionals were asked to respond whether abortion services were commonly, sometimes or rarely used by urban poor, urban non-poor, rural poor and rural non-poor for a range of service providers.

- Sampling strategy

To obtain a nationally representative sample of Health Professionals, the sampling strategy aimed at a proportionate provincial representation based on population distribution of the four provinces. The selection criteria included exposure to PAC, research/interest in induced abortion and provincial representation. Key informants were informed of the selection criteria.

The targeted sample size was later increased to 154 health professionals when other experienced individuals were identified during the data collection phase. Two thirds of respondents were from each of the two larger provinces (Punjab and Sindh) and the remaining third from the two smaller provinces (KPK, Balochistan). Over three quarters of the respondents were gynaecologists from either teaching or non-teaching tertiary care hospitals. 


\section{Annex 9: Social and health indicators of study districts-2002}

\begin{tabular}{|c|c|c|c|c|c|c|c|c|c|c|}
\hline Prov. Name & District Name & \begin{tabular}{|c} 
District \\
Population \\
1998
\end{tabular} & $\begin{array}{c}\text { Females } \\
\text { age } \\
15-49 \text { in } \\
\text { total } \\
\text { population }\end{array}$ & $\begin{array}{c}\text { Sex Ratio } \\
\text { (males } \\
\text { per } 100 \\
\text { females) }\end{array}$ & $\begin{array}{l}\text { Pop. } \\
\text { Density } \\
\text { Per } \\
\text { SQ.KM. }\end{array}$ & $\begin{array}{l}\text { Total } \\
\text { Fertility } \\
\text { Rate }\end{array}$ & $\begin{array}{c}\text { Contraceptive } \\
\text { Prevalence } \\
\text { Rate (\%) }\end{array}$ & $\begin{array}{l}\% \text { of } \\
\text { urban } \\
\text { Pop. }\end{array}$ & \begin{tabular}{|c} 
Literacy \\
Ratio \\
$(10+)$
\end{tabular} & $\begin{array}{c}\text { Average } \\
\text { Household } \\
\text { Size }\end{array}$ \\
\hline \multirow{22}{*}{ Punjab } & Attock & \begin{tabular}{|l}
1274935 \\
\end{tabular} & 307259 & 99.6 & 186 & 4.1 & 19 & 21.3 & 49.3 & \begin{tabular}{|l}
6.1 \\
\end{tabular} \\
\hline & Bahawalnagar & 2061447 & 455580 & 107.4 & 232.2 & 4.8 & 24.1 & 19.1 & 35.1 & 6.7 \\
\hline & Bahawalpur & 2433091 & 520681 & 110.8 & 98 & 5 & 25.5 & 27.3 & 34.7 & 6.8 \\
\hline & Bhakkar & 1051456 & 229217 & 107.1 & 129 & 4.7 & 14.1 & 16 & 34.2 & 6.6 \\
\hline & Gujranwala & 3400940 & 748207 & 108.6 & 939 & 4.9 & 40 & 50.5 & 56.3 & 7.5 \\
\hline & Hafizabad & 832980 & 183256 & 108.4 & 351.9 & 4.9 & 25.9 & 18.8 & 50.5 & 7.1 \\
\hline & Jhang & 2834545 & 623600 & 108.4 & 321.8 & 4.4 & 19 & 23.4 & 37.1 & 6.5 \\
\hline & Jhelum & 936957 & 224870 & 99.8 & 261.2 & 3.8 & 39 & 27.7 & 68.9 & 6.1 \\
\hline & Kasur & 2375875 & 491806 & 109.9 & 594.7 & 5 & 39 & 22.8 & 36.2 & 7 \\
\hline & Khanewal & 2068490 & 455068 & 107.7 & 475.6 & 5 & 18.7 & 17.6 & 40 & 7 \\
\hline & Lahore & 6318745 & 1484905 & 111.3 & 3565.9 & 4.4 & 53 & 82.4 & 64.6 & 7.1 \\
\hline & Layyah & 1120951 & 237642 & 106.8 & 178.2 & 5.8 & 32 & 13 & 38.7 & 7.3 \\
\hline & Mianwali & 1056620 & 243023 & 100.8 & 180.9 & 4.7 & 20.2 & 20.8 & 42.8 & 7.1 \\
\hline & Multan & 3116851 & 685707 & 110.4 & 837.9 & 5 & 33 & 42.2 & 43.4 & 7.1 \\
\hline & Muzafargarh & 2635903 & 535088 & 108.7 & 319.5 & 5.5 & 13.8 & 12.9 & 28.4 & 7.3 \\
\hline & Narowal & 1265097 & 278321 & 101.2 & 541.3 & 4.7 & 38.7 & 12.2 & 52.7 & 7.4 \\
\hline & Okara & 2232992 & 484559 & 109.6 & 510.2 & 4.6 & 30 & 23 & 37.8 & 6.5 \\
\hline & Rahim yar Khan & 3141053 & 665903 & 108.8 & 264.4 & 5 & 19.2 & 19.6 & 33.1 & 7.5 \\
\hline & Rajanpur & 1103618 & 222931 & 111.1 & 89.6 & 5.7 & 11.8 & 14.5 & 20.7 & 7.3 \\
\hline & Sahiwal & 1843194 & 414719 & 107.2 & 575.8 & 4.6 & 23.8 & 16.4 & 44 & 6.9 \\
\hline & Sialkot & 2723481 & 615507 & 105.2 & 903 & 4.7 & 52 & 26.2 & 59 & 7.3 \\
\hline & Toba teksingh & 1621593 & 364858 & 105.3 & 498.6 & 4.6 & 20 & 18.8 & 50.5 & 7.1 \\
\hline \multirow{8}{*}{ Sindh } & Hyderabad & 2891488 & 667934 & 109.5 & 523.9 & 4.8 & 34.6 & 50.8 & 44.3 & 6 \\
\hline & Karachi & 2277931 & 567205 & 111.4 & 33013.5 & 3.9 & 51.6 & 100 & 76 & 6.8 \\
\hline & Khairpur & 1546587 & 332516 & 110.1 & 97.2 & 5.3 & 19.9 & 23.6 & 35.5 & 6 \\
\hline & Larkana & 1927066 & 423955 & 106.4 & 259.6 & 5.1 & 14 & 28.9 & 35 & 5.9 \\
\hline & Mirpurkhas & 905935 & 199306 & 108.3 & 309.7 & 4.7 & 20 & 33.1 & 36 & 6.1 \\
\hline & Nawabshah & 1071533 & 242166 & 107.7 & 238 & 5.4 & 15.4 & 26.4 & 34.1 & 6 \\
\hline & Sanghar & 1453028 & 313854 & 110.4 & 135.4 & 4.9 & 15.1 & 22.8 & 30.9 & 6.4 \\
\hline & Sukkur & 908373 & 198025 & 113.7 & 175.9 & 5 & 24.5 & 50.5 & 46.6 & 6.5 \\
\hline \multirow{7}{*}{ KPK } & Abbotabad & 880666 & 208718 & 100.2 & 447.7 & 4.7 & 29.1 & 17.9 & 56.6 & 6.4 \\
\hline & Charsada & 1022364 & 215719 & 107.9 & 1026.5 & 5 & 23 & 18.9 & 31.1 & 8 \\
\hline & Dera Ismail Khan & 852995 & 176570 & 111.1 & 116.4 & 5.1 & 12 & 14.8 & 31.3 & 7.5 \\
\hline & Malakand & 452291 & 92267 & 106.8 & 475 & 5.1 & 19.5 & 9.5 & 39.5 & 9.1 \\
\hline & Mardan & 1460100 & 311001 & 106.6 & 894.7 & 5.7 & 15.2 & 20.2 & 36.5 & 8.4 \\
\hline & Peshawar & 2019118 & 434110 & 110.8 & 1606.3 & 4.9 & 35.9 & 48.7 & 41.8 & 8.5 \\
\hline & Swat & 1257602 & 264096 & 106.3 & 235.6 & 4.9 & 30.8 & 13.8 & 28.7 & 8.8 \\
\hline \multirow{2}{*}{ Balochistan } & Kharan & 206909 & 44692 & 107.6 & 4.3 & 5.6 & 15.7 & 13.4 & 15.1 & 5.8 \\
\hline & Pishin & 367183 & 75640 & 114.9 & 47 & 8 & 10.8 & 6.3 & 31.1 & 6.8 \\
\hline
\end{tabular}

\footnotetext{
Source: Population data sheet 1998 Census. NIPS
} 


\section{Annex 10: Standard errors and 95\% confidence intervals for major tables}

Table A4: Mean numbers of annual PAC patients treated as out and in-patients, by health sector and type of facility, Health Facilities Survey, 2012

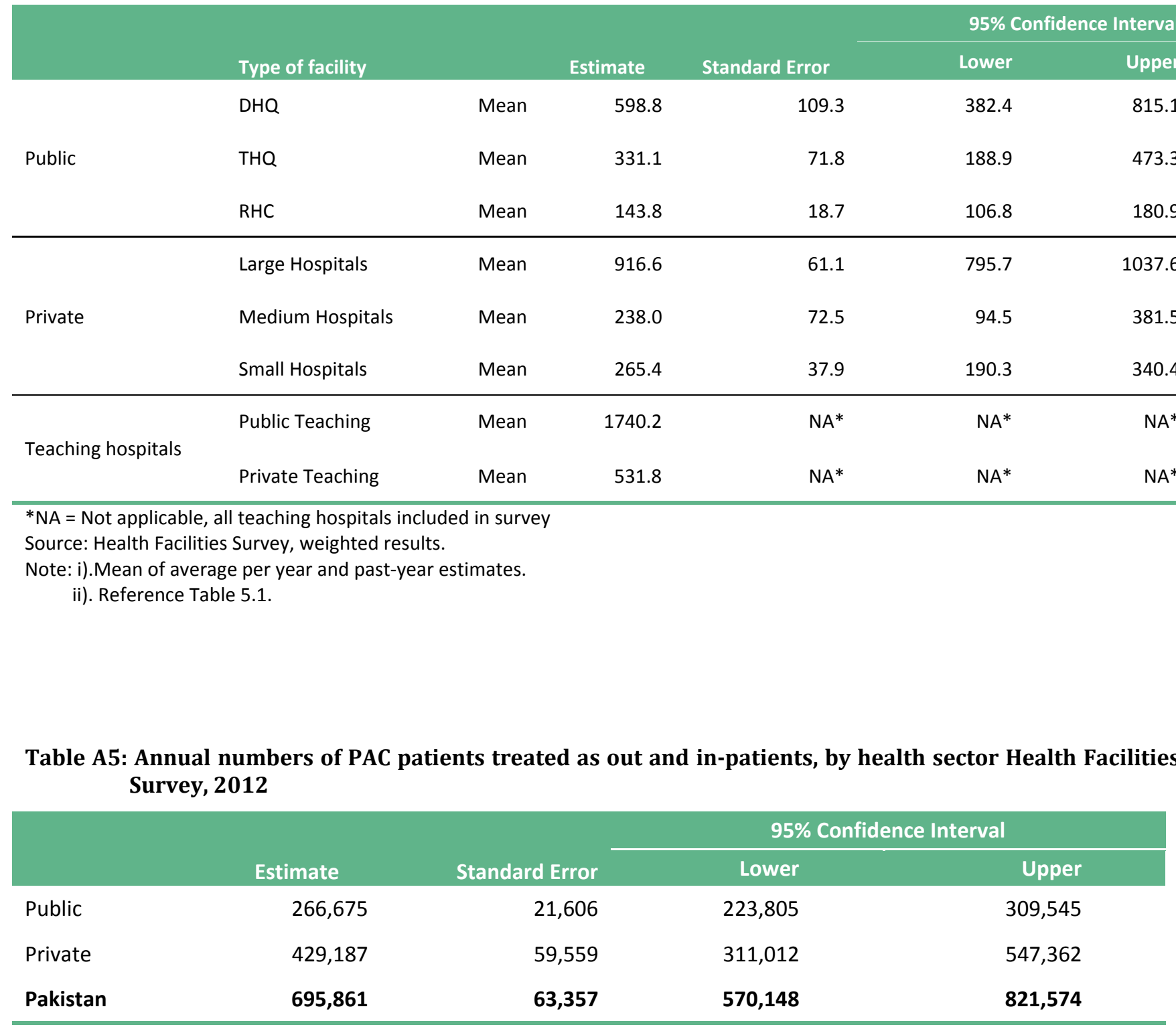

Source: Health Facilities Survey, weighted results.

Note: i). Mean of average per year and past-year estimates

ii). Reference Figure 5.1. 
Table A6: Annual number of post-abortion complications (out and in-patients) cases treated in health facilities, by province, Health Facilities Survey 2012

\begin{tabular}{|c|c|c|c|c|}
\hline & & & $95 \%$ Con & \\
\hline & Estimate & Standard Error & Lower & Upper \\
\hline Punjab & 416,433 & 69,453 & 278,624 & 554,242 \\
\hline Sindh & 174,908 & 35,278 & 104,909 & 244,907 \\
\hline KPK & 57,159 & 20,531 & 16,421 & 97,897 \\
\hline Balochistan & 47,361 & 16,958 & 13,712 & 81,010 \\
\hline Pakistan & 695,861 & 63,357 & 570,148 & 821,574 \\
\hline
\end{tabular}

Source: Health Facilities Survey, weighted results.

Note: i). Mean of average per year and past-year estimates.

ii). Reference Figure 5.2. 


\section{Annex 11: Graphical presentation and narrative of the framework approach used in the anallysis.}

Data analysis was carried out using the following five stages of framework approach ${ }^{53}$.

As shown in the figure the first stage is "Familiarization" in which the researchers immerse themselves into the raw data, especially listening to transcripts, reading and translating transcripts, analysing notes etc. This is in order to list key ideas and recurrent themes.

In the second stage, a thematic framework is "identified". This is done using the aims and objectives of the study as well issues, concepts and themes brought up by the respondents.

The third stage involves "indexing" where the framework is applied to all the text in the data through annotation of numerical codes.

The fourth stage is "charting" where the data is synthesized and rearranged according to how they relate to the thematic framework.

The final stage in the framework approach is "mapping and interpretation", when the organized data is used to define concepts, map the phenomena, find associations between themes and come up with interpretations.

This graphical presentation is illustrated in the figure below:

Five stages of data analysis in the framework approach (Pope et Al, 2000)

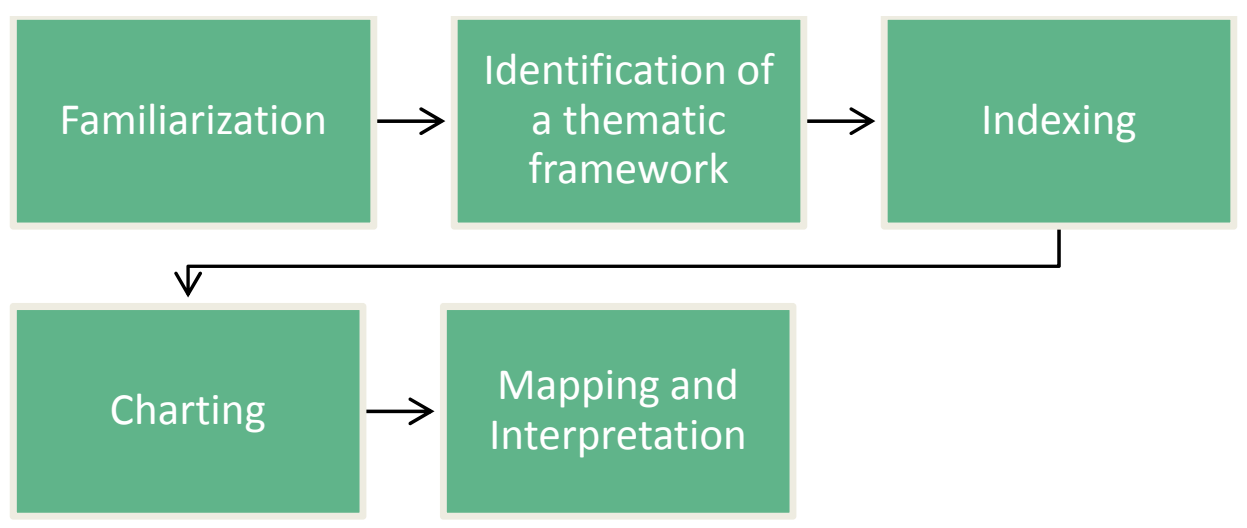

